\title{
Spectral asymptotics for large skew-symmetric perturbations of the harmonic oscillator
}

\author{
Isabelle Gallagher \\ Institut de Mathématiques de Jussieu \\ Université de Paris 7 \\ Case 7012, 2 place Jussieu \\ 75251 Paris Cedex 05, France \\ gallagher@math.jussieu.fr
}

\author{
Thierry Gallay \\ Institut Fourier \\ Université de Grenoble I \\ BP 74 \\ 38402 Saint-Martin-d'Hères, France \\ Thierry.Gallay@ujf-grenoble.fr
}

\author{
Francis Nier \\ IRMAR \\ Université de Rennes 1 \\ Campus de Beaulieu \\ 35042 Rennes, France \\ Francis.Nier@univ-rennes1.fr
}

\begin{abstract}
Originally motivated by a stability problem in Fluid Mechanics, we study the spectral and pseudospectral properties of the differential operator $H_{\epsilon}=-\partial_{x}^{2}+x^{2}+i \epsilon^{-1} f(x)$ on $L^{2}(\mathbb{R})$, where $f$ is a real-valued function and $\epsilon>0$ a small parameter. We define $\Sigma(\epsilon)$ as the infimum of the real part of the spectrum of $H_{\epsilon}$, and $\Psi(\epsilon)^{-1}$ as the supremum of the norm of the resolvent of $H_{\epsilon}$ along the imaginary axis. Under appropriate conditions on $f$, we show that both quantities $\Sigma(\epsilon), \Psi(\epsilon)$ go to infinity as $\epsilon \rightarrow 0$, and we give precise estimates of the growth rate of $\Psi(\epsilon)$. We also provide an example where $\Sigma(\epsilon) \gg \Psi(\epsilon)$ if $\epsilon$ is small. Our main results are established using variational "hypocoercive" methods, localization techniques and semiclassical subelliptic estimates.
\end{abstract}

\section{Introduction}

In many evolution equations arising in Mathematical Physics, one encounters the situation where the generator of the evolution can be written as a sum of a dissipative and a conservative operator which do not commute with each other. In such a case the conservative term can affect and sometimes enhance the dissipative effects or the regularizing properties of the whole system. For instance, if the system has a globally attracting equilibrium, the rate of convergence towards this steady state can strongly depend on the nature and the size of the conservative terms. Typical examples illustrating such an interplay between diffusion and transport are the kinetic Fokker-Planck equation [13], and the Boltzmann equation [5]; see also [24] for a comprehensive study of these phenomena at a more abstract level.

In this paper we study a simple linear system which fits into this general framework. Given a small parameter $\epsilon>0$ and a smooth, bounded function $f: \mathbb{R} \rightarrow \mathbb{R}$, we consider the differential operator

$$
H_{\epsilon}=-\partial_{x}^{2}+x^{2}+\frac{i}{\epsilon} f(x), \quad x \in \mathbb{R}
$$


acting on the Hilbert space $X=L^{2}(\mathbb{R})$, with domain $\mathcal{D}=\left\{u \in H^{2}(\mathbb{R}) ; x^{2} u \in L^{2}(\mathbb{R})\right\}$. Clearly $H_{\epsilon}$ is a bounded, skew-symmetric perturbation of the harmonic oscillator $H_{\infty}=-\partial_{x}^{2}+x^{2}$. Our goal is to compute the decay rate in time of the solutions to the evolution equation

$$
\frac{\mathrm{d} u}{\mathrm{~d} t}=-H_{\epsilon} u, \quad u(0)=u_{0} \in X .
$$

As we shall see, the solutions to (1.2) decay to zero at least like $e^{-t}$ as $t \rightarrow+\infty$, but the actual convergence rate strongly depends on the value of $\epsilon$ and the detailed properties of $f$, due to the interaction between the symmetric (dissipative) and the skew-symmetric (conservative) part of the generator $-H_{\epsilon}$.

Our initial motivation for this study is a specific problem in Fluid Mechanics which we now briefly describe. As is explained in [6, 7], to investigate the long-time behavior of solutions to the two-dimensional Navier-Stokes equation, it is convenient to use the the vorticity formulation. In self-similar variables, the system reads:

$$
\frac{\partial \omega}{\partial t}+u \cdot \nabla \omega=\Delta \omega+\frac{1}{2} x \cdot \nabla \omega+\omega, \quad x \in \mathbb{R}^{2}, \quad t \geq 0,
$$

where $\omega(x, t) \in \mathbb{R}$ is the vorticity distribution and $u(x, t) \in \mathbb{R}^{2}$ is the divergence-free velocity field obtained from $\omega$ via the Biot-Savart law. Equation (1.3) has a family of stationary solutions, called Oseen vortices, of the form $\omega=\alpha G$ where $G(x)=(4 \pi)^{-1} e^{-|x|^{2} / 4}$ and $\alpha \in \mathbb{R}$ is a free parameter (the circulation Reynolds number). It turns out that the linearization of (1.3) at $\alpha G$ has the same form as (1.2), in the sense that the generator can be written as a difference $\mathcal{L}-\alpha \Lambda$, where $\mathcal{L}$ is a self-adjoint operator in the weighted space $L^{2}\left(\mathbb{R}^{2}, G^{-1} \mathrm{x}\right)$ and $\Lambda$ is a skew-symmetric perturbation. The analogy goes even further if we conjugate $\mathcal{L}-\alpha \Lambda$ with the Gaussian weight $G^{1 / 2}$ and if we neglect a nonlocal, lower-order term in the perturbation $\Lambda$. The linearized operator then becomes

$$
\widetilde{H}_{\alpha}=-\Delta+\frac{|x|^{2}}{16}-\frac{1}{2}+\alpha \tilde{f}(x) \partial_{\theta}, \quad x \in \mathbb{R}^{2},
$$

where $\partial_{\theta}=x_{1} \partial_{2}-x_{2} \partial_{1}$ and $\tilde{f}(x)=\left(2 \pi|x|^{2}\right)^{-1}\left(1-e^{-|x|^{2} / 4}\right)$. The operator $H_{\epsilon}$ in (1.1) is a onedimensional analog of $\widetilde{H}_{\alpha}$, and the limit $\epsilon \rightarrow 0$ corresponds to the fast rotation limit $\alpha \rightarrow \infty$. Remark that, in this particular example, the function $\tilde{f}$ has a unique critical point located at the origin, and decreases to zero like $|x|^{-2}$ as $|x| \rightarrow \infty$.

The aim of this paper is to study the spectral and pseudospectral properties of the linear operator $H_{\epsilon}$ in the limit $\epsilon \rightarrow 0$. Besides the specific motivations explained above, this question has its own interest from a mathematical point of view, and turns out to be relatively complex. We have to deal with a non-self-adjoint problem of (almost) semiclassical type which exhibits a competition between various microlocal models at different scales, depending on the structure of the critical points and the decay rate at infinity of the function $f$. In particular, unlike in the self-adjoint case, or even in some non-self-adjoint problems such as the kinetic Fokker-Planck operator (see [10, 13, 14]), the pseudospectral estimates are not monotone with respect to the imaginary part of the spectral parameter. Nevertheless, the model (1.1) is simple enough so that the analysis can be pushed quite far, and we believe that our results give a good idea of the phenomena that can be expected to occur in more general situations. We also mention that the spectral theory of non-self-adjoint operators, especially in the semiclassical limit, is a topic of current interest [23, 3, 4, 22].

We start with a few basic observations concerning the operator $H_{\epsilon}$. As is well known the limiting operator $H_{\infty}=-\partial_{x}^{2}+x^{2}$ is self-adjoint in $L^{2}(\mathbb{R})$ with compact resolvent, and its spectrum 
is a sequence of simple eigenvalues $\left\{\lambda_{n}^{0}\right\}_{n \in \mathbb{N}}$, where $\lambda_{n}^{0}=2 n+1$. By classical perturbation theory [17], it follows that $H_{\epsilon}$ has a compact resolvent for any $\epsilon>0$, and that its spectrum is again a sequence of (simple) eigenvalues $\left\{\lambda_{n}(\epsilon)\right\}_{n \in \mathbb{N}}$, with $\operatorname{Re}\left(\lambda_{n}(\epsilon)\right) \rightarrow+\infty$ as $n \rightarrow \infty$. Moreover, it is clear from (1.1) that the numerical range

$$
\Theta\left(H_{\epsilon}\right)=\left\{\left\langle H_{\epsilon} u, u\right\rangle_{L^{2}} \in \mathbb{C} ; u \in \mathcal{D},\|u\|_{L^{2}}=1\right\}
$$

is contained in the region $\mathcal{R}_{\epsilon} \subset \mathbb{C}$ defined by

$$
\mathcal{R}_{\epsilon}=\{\lambda \in \mathbb{C} ; \operatorname{Re}(\lambda) \geq 1, \epsilon \operatorname{Im}(\lambda) \in \overline{f(\mathbb{R})}\} .
$$

In particular we have $\lambda_{n}(\epsilon) \in \mathcal{R}_{\epsilon}$ for all $n \in \mathbb{N}$ and all $\epsilon>0$. The operator $H_{\epsilon}$ is therefore sectorial, and $H_{\epsilon}-1$ is maximal accretive, see Section V-3.10 in [17. By the Lumer-Phillips theorem, it follows that

$$
\left\|e^{-t H_{\epsilon}}\right\| \leq e^{-t}, \quad \text { for all } t \geq 0 .
$$

Here and in the sequel we denote by $\|A\|$ the norm of a bounded linear operator $A$ in $L^{2}(\mathbb{R})$, and by $R_{\mathrm{sp}}(A)$ its spectral radius.

Since we want to compute the decay rate of the solutions to (1.2), we are interested in locating the real part of the spectrum of $H_{\epsilon}$. We thus define:

$$
\Sigma(\epsilon)=\inf \operatorname{Re}\left(\sigma\left(H_{\epsilon}\right)\right)=\min _{n \in \mathbb{N}} \operatorname{Re}\left(\lambda_{n}(\epsilon)\right) .
$$

We also introduce a related pseudospectral quantity, which will play an important role in this work:

$$
\Psi(\epsilon)=\left(\sup _{\lambda \in \mathbb{R}}\left\|\left(H_{\epsilon}-i \lambda\right)^{-1}\right\|\right)^{-1}
$$

It is easy to see that $\Sigma(\epsilon) \geq \Psi(\epsilon) \geq 1$. Indeed, for any $\lambda \in \mathbb{R}$, we have

$$
\frac{1}{\operatorname{dist}\left(i \lambda, \sigma\left(H_{\epsilon}\right)\right)}=R_{\mathrm{sp}}\left(\left(H_{\epsilon}-i \lambda\right)^{-1}\right) \leq\left\|\left(H_{\epsilon}-i \lambda\right)^{-1}\right\| \leq \frac{1}{\operatorname{dist}\left(i \lambda, \Theta\left(H_{\epsilon}\right)\right)},
$$

see e.g. Problem III-6.16 and Theorem V-3.2 in [17]. Taking the supremum over $\lambda \in \mathbb{R}$ and using the inclusion $\Theta\left(H_{\epsilon}\right) \subset \mathcal{R}_{\epsilon}$, we thus find

$$
\frac{1}{\Sigma(\epsilon)} \leq \frac{1}{\Psi(\epsilon)} \leq \sup _{\lambda \in \mathbb{R}} \frac{1}{\operatorname{dist}\left(i \lambda, \mathcal{R}_{\epsilon}\right)}=1
$$

which is the desired result. Elementary relations between $\Sigma(\epsilon), \Psi(\epsilon)$ and the norm of the semigroup $e^{-t H_{\epsilon}}$ can also be deduced from the following general lemma:

Lemma 1.1 Let $A$ be a maximal accretive operator in a Hilbert space $X$, with numerical range contained in the sector $\left\{z \in \mathbb{C} ;|\arg z| \leq \frac{\pi}{2}-2 \alpha\right\}$ for some $\alpha \in\left(0, \frac{\pi}{4}\right]$. Assume that $A$ is invertible and let

$$
\Sigma=\inf \operatorname{Re}(\sigma(A))>0, \quad \text { and } \quad \Psi=\left(\sup _{\lambda \in \mathbb{R}}\left\|(A-i \lambda)^{-1}\right\|\right)^{-1} .
$$

Then the following holds:

i) If there exist $C \geq 1$ and $\mu>0$ such that $\left\|e^{-t A}\right\| \leq C e^{-\mu t}$ for all $t \geq 0$, then

$$
\Sigma \geq \mu, \quad \text { and } \quad \Psi \geq \frac{\mu}{1+\log (C)} .
$$


ii) For any $\mu \in(0, \Sigma)$, we have $\left\|e^{-t A}\right\| \leq C(A, \mu) e^{-\mu t}$ for all $t \geq 0$, where

$$
C(A, \mu)=\frac{1}{\pi \tan \alpha}(\mu N(A, \mu)+2 \pi), \quad \text { and } \quad N(A, \mu)=\sup _{\lambda \in \mathbb{R}}\left\|(A-\mu-i \lambda)^{-1}\right\| .
$$

iii) If moreover $\mu \in(0, \Psi)$, the quantity $N(A, \mu)$ is not larger than $(\Psi-\mu)^{-1}$.

For completeness, we give a proof of this lemma in Appendix $\mathrm{A}$. We also mention that a similar result holds for some maximal accretive operators with $\alpha=0$, in which case the constant $C(A, \mu)$ has a different expression, see [13] or [10] for a detailed study of Fokker-Planck operators which enter in this category. In the case when $A=H_{\epsilon}$, we have of course $\Sigma=\Sigma(\epsilon), \Psi=\Psi(\epsilon)$, and the angle $\alpha$ satisfies $\tan (2 \alpha)=\epsilon\|f\|_{L^{\infty}}^{-1}$, see (1.5), (1.6). Thus $\alpha=\mathcal{O}(\epsilon)$ as $\epsilon \rightarrow 0$.

Lemma 1.1 can be used in particular to illustrate the pseudospectral nature of the quantity $\Psi(\epsilon)$ defined in (1.8). For semiclassical operators of the form $P_{\epsilon}=p\left(x, \epsilon \partial_{x}\right)$, the pseudospectrum is usually defined as the set of all $z \in \mathbb{C}$ such that $\epsilon^{N}\left\|\left(P_{\epsilon}-z\right)^{-1}\right\| \rightarrow \infty$ as $\epsilon \rightarrow 0$ for all $N \in \mathbb{N}$, see e.g. [19]. Instead of defining the pseudospectrum as a subset of $\mathbb{C}$, we find it convenient to introduce here a more flexible notion:

Definition 1.2 Let $\left(\omega_{\epsilon}\right)_{\epsilon \in(0,1]}$ be a family of complex domains, i.e. $\omega_{\epsilon} \subset \mathbb{C}$ for all $\epsilon \in(0,1]$. We say that $\omega_{\epsilon}$ meets the pseudospectrum of $H_{\epsilon}$ as $\epsilon \rightarrow 0$ if

$$
\lim _{\epsilon \rightarrow 0} \epsilon^{N} \sup _{z \in \omega_{\epsilon}}\left\|\left(H_{\epsilon}-z\right)^{-1}\right\|=+\infty, \quad \text { for all } N \in \mathbb{N} .
$$

On the contrary, we say that $\omega_{\epsilon}$ avoids the pseudospectrum of $H_{\epsilon}$ as $\epsilon \rightarrow 0$ if there exists $N \in \mathbb{N}$ such that

$$
\sup _{z \in \omega_{\epsilon}}\left\|\left(H_{\epsilon}-z\right)^{-1}\right\|=\mathcal{O}\left(\epsilon^{-N}\right), \quad \text { as } \epsilon \rightarrow 0 .
$$

If we apply this definition in the case when $\omega_{\epsilon}=\left\{z \in \mathbb{C} ; \operatorname{Re}(z) \leq \mu_{\epsilon}\right\}$, we arrive at very different conclusions depending on whether $\mu_{\epsilon} \ll \Psi(\epsilon)$ or $\mu_{\epsilon} \gg \Psi(\epsilon)$. Indeed, using Lemma 1.1 and Definition 1.2, we obtain the following result, whose proof is again postponed to Appendix $\mathrm{A}$ :

\section{Lemma 1.3}

i) For any $\kappa \in(0,1)$, the domain $\{\operatorname{Re}(z) \leq \kappa \Psi(\epsilon)\}$ avoids the pseudospectrum of $H_{\epsilon}$ as $\epsilon \rightarrow 0$.

ii) If $\mu_{\epsilon} \gg \Psi(\epsilon)\left(1+\log \Psi(\epsilon)+\log \left(\epsilon^{-1}\right)\right)$ in the sense that the ratio goes to $+\infty$ as $\epsilon \rightarrow 0$, then the domain $\left\{\operatorname{Re}(z) \leq \mu_{\epsilon}\right\}$ meets the pseudospectrum of $H_{\epsilon}$ as $\epsilon \rightarrow 0$.

The main purpose of the present work is to investigate the behavior of both quantities $\Sigma(\epsilon)$ and $\Psi(\epsilon)$ as $\epsilon \rightarrow 0$. In particular, we shall give precise estimates on $\Psi(\epsilon)$ under rather general assumptions on the function $f$. Computing $\Sigma(\epsilon)$ is a more delicate task, and we shall restrict ourselves to a specific example which shows that $\Sigma(\epsilon)$ can be much larger than $\Psi(\epsilon)$ when $\epsilon$ is small. This can be expected because $H_{\epsilon}$ is highly non-self-adjoint in this regime (we recall that $\Sigma=\Psi$ for self-adjoint operators).

If the function $f: \mathbb{R} \rightarrow \mathbb{R}$ is not identically constant, it is rather straightforward to verify that $\Sigma(\epsilon) \geq \Psi(\epsilon)>1$ for all $\epsilon>0$, see Lemma 2.1 below. In view of Lemma 1.1, we conclude that the decay properties of the semigroup $e^{-t H_{\epsilon}}$ are always enhanced by the skew-symmetric perturbation $i \epsilon^{-1} f(x)$, if $f$ is not constant. It is therefore very natural to ask under which conditions on $f$ the decay rate can be made arbitrarily large by choosing $\epsilon$ sufficiently small. If we use the pseudospectral quantity $\Psi(\epsilon)$ to measure the decay, the complete answer is given by the following proposition, which is proved in Section 2 . 
Proposition 1.4 Assume that $f \in L^{\infty}(\mathbb{R}) \cap C^{0}(\mathbb{R})$. If all level sets of $f$ have empty interior, then $\Psi(\epsilon) \rightarrow \infty$ as $\epsilon \rightarrow 0$. In the converse case, $\Psi(\epsilon)$ is uniformly bounded for all $\epsilon>0$.

We suspect that Proposition 1.4 remains true if we replace $\Psi(\epsilon)$ by $\Sigma(\epsilon)$, but at the present time there is no proof that $\Sigma(\epsilon)$ remains bounded if $f$ is constant on some nonempty open interval.

We now turn to more quantitative versions of Proposition 1.4, which specify the growth rate of $\Psi(\epsilon)$ or $\Sigma(\epsilon)$ as $\epsilon \rightarrow 0$. Our first result in this direction will be obtained using a variational method recently developed by C. Villani under the name of "hypocoercivity" [24]. This approach applies (in particular) to linear operators of the form $L=A^{*} A+B$ in a Hilbert space $X$, where $B$ is skew-symmetric. Under certain conditions, it allows to compare the spectral properties of $L$ with those of the self-adjoint operator $\hat{L}=A^{*} A+C^{*} C$, where $C=[A, B]$. It is clear that our problem fits into this general framework: if we set $X=L^{2}(\mathbb{R}, \mathbb{C}), A=\partial_{x}+x$, and $B=(i / \epsilon) f(x)$, we see that $H_{\epsilon}-1=A^{*} A+B$. Since $C=[A, B]=(i / \epsilon) f^{\prime}(x)$, the associated self-adjoint operator $\hat{H}_{\epsilon}$ defined by $\hat{H}_{\epsilon}-1=A^{*} A+C^{*} C$ has the explicit form

$$
\hat{H}_{\epsilon}=-\partial_{x}^{2}+x^{2}+\frac{1}{\epsilon^{2}} f^{\prime}(x)^{2}, \quad x \in \mathbb{R} .
$$

The point is that the spectral properties of $\hat{H}_{\epsilon}$ in the limit $\epsilon \rightarrow 0$ are rather easy to study using semiclassical techniques, see e.g. Lemma 1.7 below. Once the spectrum of $\hat{H}_{\epsilon}$ is known, Villani's method allows to deduce useful information on the original operator $H_{\epsilon}$. A great interest of this approach is that it can be easily adapted to nonlinear problems [24, 25].

Following closely the proof of the "basic hypocoercivity result" in [24, Section 4], we obtain our first main result:

Theorem 1.5 Assume that $f \in C^{3}(\mathbb{R})$ satisfies $f^{\prime \prime}, f^{\prime \prime \prime} \in L^{\infty}(\mathbb{R})$, and that there exist $M_{1}>0$ and $\nu \in(0,1 / 2]$ such that

$$
\left\langle\hat{H}_{\epsilon} u, u\right\rangle_{L^{2}}=\int_{\mathbb{R}}\left(\left|\partial_{x} u\right|^{2}+x^{2}|u|^{2}+\frac{1}{\epsilon^{2}} f^{\prime}(x)^{2}|u|^{2}\right) x \geq \frac{M_{1}}{\epsilon^{2 \nu}}\|u\|_{L^{2}}^{2},
$$

for all $u \in \mathcal{D}$ and all $\epsilon \in(0,1]$. Then there exists $M_{2}>0$ such that, for all $\epsilon \in(0,1]$,

$$
\Sigma(\epsilon) \geq \frac{M_{2}}{\epsilon^{\nu}}, \quad \text { and } \quad \Psi(\epsilon) \geq \frac{M_{2}}{\epsilon^{\nu} \log (2 / \epsilon)} .
$$

Remark that we do not suppose in Theorem 1.5 that $f$ is bounded, but only that $f^{\prime \prime}$ and $f^{\prime \prime \prime}$ are. Under these more general assumptions, the perturbation $H_{\epsilon}-H_{\infty}$ is relatively bounded with respect to $H_{\infty}$, but not necessarily relatively compact. To test the conclusion on a concrete example, consider the simple case when $f(x)=x^{2}$. Then we have $H_{\epsilon}=-\partial_{x}^{2}+(1+i / \epsilon) x^{2}$, so that $\Sigma(\epsilon)=\operatorname{Re}\left((1+i / \epsilon)^{1 / 2}\right)=\mathcal{O}\left(\epsilon^{-1 / 2}\right)$ as $\epsilon \rightarrow 0$. On the other hand, $\hat{H}_{\epsilon}=-\partial_{x}^{2}+\left(1+4 / \epsilon^{2}\right) x^{2}$, hence $\inf \left(\sigma\left(\hat{H}_{\epsilon}\right)\right)=\left(1+4 / \epsilon^{2}\right)^{1 / 2}$ so that (1.11) is satisfied with $\nu=1 / 2$. Thus the lower bound (1.12) for $\Sigma(\epsilon)$ is optimal, and one can also check that the estimate for $\Psi(\epsilon)$ is optimal except for the logarithmic correction. However, as we shall see, the results given by Theorem 1.5 are not always optimal, especially when $f^{\prime}(x)$ decays algebraically as $|x| \rightarrow \infty$.

Motivated by our original example (1.4), we next consider a specific class of nonlinearities with a prescribed behavior at infinity.

Hypothesis 1.6 We assume that $f \in C^{3}(\mathbb{R}, \mathbb{R})$ has the following properties:

i) All critical points of $f$ are non-degenerate; i.e., $f^{\prime}(x)=0$ implies $f^{\prime \prime}(x) \neq 0$. 
ii) There exist positive constants $C$ and $k$ such that, for all $x \in \mathbb{R}$ with $|x| \geq 1$,

$$
\left|\partial_{x}^{\ell}\left(f(x)-\frac{1}{|x|^{k}}\right)\right| \leq \frac{C}{|x|^{k+\ell+1}}, \quad \text { for } \ell=0,1,2,3 .
$$

Loosely speaking, we consider Morse functions which are bounded together with their derivatives up to third order, and which behave like $|x|^{-k}$ as $|x| \rightarrow \infty$. This is not the most general class that we can treat with our methods, but it already contains many interesting examples, including the original problem (1.4). The assumption that $f^{\prime}(x)$ decays algebraically as $|x| \rightarrow \infty$ is very important: as we shall see, the decay rate of the semigroup $e^{-t H_{\epsilon}}$ is determined not only by the structure of the critical points of $f$, but also by the behavior of $f$ near infinity, and under hypothesis (1.13) there is a competition between both regimes which is one of the main interests of our problem.

Under Hypothesis 1.6, it is rather straightforward to estimate the lowest eigenvalue of the self-adjoint operator $\hat{H}_{\epsilon}$. The result is:

Lemma 1.7 If $f$ satisfies Hypothesis [1.6, there exists $M_{3} \geq 1$ such that, for all $\epsilon \in(0,1]$,

$$
\frac{1}{M_{3} \epsilon^{2 \nu}} \leq \inf \sigma\left(\hat{H}_{\epsilon}\right) \leq \frac{M_{3}}{\epsilon^{2 \nu}}, \quad \text { where } \quad \nu=\frac{1}{k+2} .
$$

For the reader's convenience, we sketch the proof of this result in Appendix $\mathrm{A}$. Since (1.14) implies (1.11) with the same value of $\nu$, Theorem 1.5] shows that $\Sigma(\epsilon), \Psi(\epsilon)$ obey the lower bounds (1.12) with $\nu=(k+2)^{-1}$ if $f$ satisfies Hypothesis 1.6. It turns out that these estimates are not optimal in the present case, neither for $\Sigma(\epsilon)$ nor for $\Psi(\epsilon)$. As is discussed in Section 3 below, this is not an intrinsic limitation of the variational method, but the proof of a general result such as Theorem 1.5 fails to take into account all specificities of $f$. Our second main result gives optimal estimates on $\Psi(\epsilon)$ under Hypothesis 1.6.

Theorem 1.8 If $f$ satisfies Hypothesis [1.6, there exists $M_{4} \geq 1$ such that, for all $\epsilon \in(0,1]$,

$$
\frac{1}{M_{4} \epsilon^{\bar{\nu}}} \leq \Psi(\epsilon) \leq \frac{M_{4}}{\epsilon^{\bar{\nu}}}, \text { where } \quad \bar{\nu}=\frac{2}{k+4} .
$$

Remark that $\bar{\nu}>\nu$, so that the lower bound in (1.15) is strictly better than in (1.12). In this paper, we shall give two different proofs of this lower bound. The first one consists in adapting the variational method that we already used in the proof of Theorem 1.5. This approach gives the lower bound in (1.15) with a logarithmic correction, as in (1.12). The second proof, which removes the logarithmic correction and also provides the upper bound in (1.15), uses rather classical techniques in the analysis of partial differential operators. The idea is first to reduce the problem to a bounded domain using a dyadic partition of unity in $\mathbb{R}$, and then to apply semiclassical subelliptic techniques as developed, for instance, in [4] and in Chap. 27 of [16].

Finally, we discuss the behavior of the spectral quantity $\Sigma(\epsilon)$. Under Hypothesis 1.6. Theorem 1.8 already provides a good lower bound on $\Sigma(\epsilon)$, via the inequality $\Sigma(\epsilon) \geq \Psi(\epsilon)$. In fact, if one believes that the analysis of small random perturbations of non-self-adjoint semiclassical operators presented in [8] can be carried over to the operator $H_{\epsilon}$, one also expects that the lower bound $\Sigma(\epsilon) \geq \Psi(\epsilon)$ is optimal for a generic $f$ in a wide class of functions. However $\Sigma(\epsilon)$ can be much larger than $\Psi(\epsilon)$ in some particular situations. This is the case for instance when $f(x)=x$, because one can show that $\Sigma(\epsilon)=\mathcal{O}\left(\epsilon^{-2}\right)$ and $\Psi(\epsilon)=\mathcal{O}\left(\epsilon^{-2 / 3}\right)$, see Section 3.2 .2 below. To conclude this paper, we consider a more interesting example which satisfies Hypothesis 1.6 and is inspired by the original problem (1.4). 
Proposition 1.9 Fix $k>0$ and assume that

$$
f(x)=\frac{1}{\left(1+x^{2}\right)^{k / 2}}, \quad x \in \mathbb{R} .
$$

Then there exists a constant $M_{5}>0$ such that the lowest real part of the spectrum satisfies, for all $\epsilon \in(0,1]$,

$$
\Sigma(\epsilon) \geq \frac{M_{5}}{\epsilon^{\nu^{\prime}}}, \quad \text { where } \quad \nu^{\prime}=\min \left\{\frac{1}{2}, \frac{2}{k+2}\right\}
$$

Since $\bar{\nu}<\nu^{\prime}$, it follows from (1.15) and (1.17) that $\Sigma(\epsilon) / \Psi(\epsilon) \rightarrow \infty$ as $\epsilon \rightarrow 0$. In particular, if we take $\mu_{\epsilon}=\frac{1}{2} M_{5} \epsilon^{-\nu^{\prime}}$, Lemma 1.3 implies that the domain $\left\{\operatorname{Re}(z) \leq \mu_{\epsilon}\right\}$ meets the pseudospectrum of $H_{\epsilon}$ as $\epsilon \rightarrow 0$ in the sense of Definition 1.2. It is also interesting to test the conclusions of Lemma 1.1 in this situation: we know from ii) that $\left\|e^{-t H_{\epsilon}}\right\| \leq C e^{-\mu_{\epsilon} t}$ for some $C \geq 1$, but it follows from i) that $C \geq e^{-1} \exp \left(\mu_{\epsilon} / \Psi(\epsilon)\right)$. Thus, although we can prove that the semigroup $e^{-t H_{\epsilon}}$ decays with an exponential rate $\mu_{\epsilon} \gg \Psi(\epsilon)$, this estimate is inadequate for small times because the prefactor $C$ is exponentially large. In contrast, if we choose $\mu_{\epsilon} \leq \kappa \Psi(\epsilon)$ for some $\kappa<1$, we can take $C=\mathcal{O}\left(\epsilon^{-1}\right)$ by Lemma 1.1-iii).

Proposition 1.9 will be proved in Section 5 below, using a complex deformation method (which exploits the analyticity properties of $f$ ) and the same localization techniques as in the proof of Theorem 1.8. We shall also give heuristical arguments, supported by accurate numerical computations, which show that the lower bound in (1.17) is optimal in the sense that the exponent $\nu^{\prime}$ cannot be improved.

The rest of this paper is organized as follows. In Section 2 we prove some general properties of $\Psi(\epsilon)$ using mainly compactness arguments. Section 3 is devoted to the proof of Theorem 1.5 using the approach developed by Villani [24]. We also show how that the variational method can be adapted to give the lower bound on $\Psi(\epsilon)$ in Theorem 1.8, up to a logarithmic correction. In Section 4, we give accurate bounds on the resolvent $\left(H_{\epsilon}-i \lambda\right)^{-1}$ for $\lambda \in \mathbb{R}$ using a dyadic decomposition of the real axis and semiclassical subelliptic estimates. In particular we prove Theorem 1.8, and we also provide a family $\left(\omega_{\epsilon}\right)_{\epsilon \in(0,1]}$ of complex domains which avoid the pseudospectrum of $H_{\epsilon}$ as $\epsilon \rightarrow 0$ according to Definition 1.2, with some non trivial $\epsilon$-dependent geometry. Finally, in Section 5, the lower bound (1.17) on $\Sigma(\epsilon)$ is proved and illustrated by numerical computations. The proofs of Lemmas 1.1, 1.3, and 1.7 are collected in Appendix A,

Acknowledgements. The authors would like to thank V. Bach, Y. Colin de Verdière, M. Hitrik, A. Joye, G. Perelman, C. Villani, S. Vu Ngoc, and M. Zworski for fruitful discussions.

\section{Compactness estimates}

In this section we establish a few general properties of the quantities $\Sigma(\epsilon)$ and $\Psi(\epsilon)$ defined in (1.7), (1.8). We first show that any nontrivial function $f$ does affect the spectrum of the operator $H_{\epsilon}$.

Lemma 2.1 Assume that $f \in L^{\infty}(\mathbb{R})$ is not a constant. Then $\Sigma(\epsilon) \geq \Psi(\epsilon)>1$ for all $\epsilon>0$.

Proof: Suppose on the contrary that $\Psi(\epsilon)=1$ for some $\epsilon>0$. Then, in view of (1.8), (1.9), there exists $\lambda_{0} \in \mathbb{R}$ such that $\left\|\left(H_{\epsilon}-i \lambda_{0}\right)^{-1}\right\|=1$. Thus, for any $n \in \mathbb{N}^{*}$, we can find $\phi_{n} \in L^{2}(\mathbb{R})$ with $\left\|\phi_{n}\right\|=1$ such that

$$
1-\frac{1}{n} \leq\left\|\left(H_{\epsilon}-i \lambda_{0}\right)^{-1} \phi_{n}\right\| \leq 1
$$


Let $\psi_{n}=\left(H_{\epsilon}-i \lambda_{0}\right)^{-1} \phi_{n}$, so that $\psi_{n} \in \mathcal{D}$ and $\phi_{n}=\left(H_{\epsilon}-i \lambda_{0}\right) \psi_{n}$. For all $n \in \mathbb{N}^{*}$ we have

$$
\left\|\psi_{n}^{\prime}\right\|^{2}+\left\|x \psi_{n}\right\|^{2}=\operatorname{Re}\left\langle\left(H_{\epsilon}-i \lambda_{0}\right) \psi_{n}, \psi_{n}\right\rangle=\operatorname{Re}\left\langle\phi_{n}, \psi_{n}\right\rangle \leq 1
$$

By Rellich's theorem, the sequence $\left\{\psi_{n}\right\}$ is compact in $L^{2}(\mathbb{R})$. Thus, after extracting a subsequence, we can assume that $\psi_{n}$ converges in $L^{2}(\mathbb{R})$ to some limit $\psi$. By construction $\|\psi\|=1$ and

$$
\left\|\psi^{\prime}\right\|^{2}+\|x \psi\|^{2} \leq \liminf _{n \rightarrow \infty}\left(\left\|\psi_{n}^{\prime}\right\|^{2}+\left\|x \psi_{n}\right\|^{2}\right) \leq 1
$$

Since $\left\|\psi^{\prime}\right\|^{2}+\|x \psi\|^{2}=\left\langle H_{\infty} \psi, \psi\right\rangle$, this inequality implies that $\psi(x)=\pi^{-1 / 4} e^{-x^{2} / 2}$ (up to a constant phase factor). Moreover, since $\psi_{n} \rightarrow \psi$ in $L^{2}(\mathbb{R})$ and $\left\|\left(H_{\epsilon}-i \lambda_{0}\right) \psi_{n}\right\| \leq 1$ for all $n$, we necessarily have $\left\|\left(H_{\epsilon}-i \lambda_{0}\right) \psi\right\| \leq 1$. But $\left(H_{\epsilon}-i \lambda_{0}\right) \psi=\psi+i\left(\epsilon^{-1} f-\lambda_{0}\right) \psi$ and therefore

$$
\left\|\left(H_{\epsilon}-i \lambda_{0}\right) \psi\right\|^{2}=\int_{\mathbb{R}}|\psi(x)|^{2}\left\{1+\left(\frac{1}{\epsilon} f(x)-\lambda_{0}\right)^{2}\right\} \mathrm{x}=1+\int_{\mathbb{R}}|\psi(x)|^{2}\left(\frac{1}{\epsilon} f(x)-\lambda_{0}\right)^{2} \mathrm{x},
$$

hence it is possible to have $\left\|\left(H_{\epsilon}-i \lambda_{0}\right) \psi\right\| \leq 1$ only if $f(x)=\epsilon \lambda_{0}$ almost everywhere.

Using similar arguments, we next prove that $\Psi(\epsilon) \rightarrow \infty$ as $\epsilon \rightarrow 0$ if and only if the level sets of $f$ have empty interior (we assume here, for simplicity, that $f$ is continuous).

Proof of Proposition 1.4; Assume first that $\Psi(\epsilon)^{-1}$ does not converge to zero as $\epsilon \rightarrow 0$. Then, according to definition (1.8), there exist a positive constant $\delta$, a sequence $\left\{\epsilon_{n}\right\}$ of positive numbers, a sequence $\left\{\lambda_{n}\right\}$ of real numbers, and a sequence $\left\{u_{n}\right\}$ of normalized vectors in $L^{2}(\mathbb{R})$ such that $\epsilon_{n} \rightarrow 0$ as $n \rightarrow \infty$ and

$$
\left\|\left(H_{\epsilon_{n}}-i \lambda_{n}\right)^{-1} u_{n}\right\| \geq \delta>0, \quad \text { for all } n \in \mathbb{N} .
$$

Let $v_{n}=\left(H_{\epsilon_{n}}-i \lambda_{n}\right)^{-1} u_{n}$, so that $v_{n} \in \mathcal{D}$ and $u_{n}=\left(H_{\epsilon_{n}}-i \lambda_{n}\right) v_{n}$. The sequence $\left\{v_{n}\right\}$ is bounded in $L^{2}(\mathbb{R})$, because $\left\|v_{n}\right\| \leq\left\|u_{n}\right\| \leq 1$ for all $n \in \mathbb{N}$. Moreover, we have the identity

$$
\left\langle u_{n}, v_{n}\right\rangle=\left\|v_{n}^{\prime}\right\|^{2}+\left\|x v_{n}\right\|^{2}+\frac{i}{\epsilon_{n}}\left\langle f v_{n}, v_{n}\right\rangle-i \lambda_{n}\left\|v_{n}\right\|^{2}
$$

Taking the real parts of both sides, we find $\left\|v_{n}^{\prime}\right\|^{2}+\left\|x v_{n}\right\|^{2} \leq\left|\left\langle u_{n}, v_{n}\right\rangle\right| \leq 1$ for all $n \in \mathbb{N}$. By Rellich's theorem, the sequence $\left\{v_{n}\right\}$ is therefore compact in $L^{2}(\mathbb{R})$. Thus, after extracting a subsequence, we can assume that $v_{n}$ converges in $L^{2}(\mathbb{R})$ to some limit $v$. By construction, $v \in H^{1}(\mathbb{R})$ and $\|v\|_{L^{2}} \geq \delta>0$.

On the other hand, multiplying both sides of (2.1) by $\epsilon_{n}$ and taking imaginary parts, we see that

$$
\left\langle f v_{n}, v_{n}\right\rangle-\epsilon_{n} \lambda_{n}\left\|v_{n}\right\|^{2}=\epsilon_{n} \operatorname{Im}\left\langle u_{n}, v_{n}\right\rangle \underset{n \rightarrow \infty}{\longrightarrow} 0 .
$$

Since $\left\langle f v_{n}, v_{n}\right\rangle \rightarrow\langle f v, v\rangle$, we conclude that

$$
\lim _{n \rightarrow \infty} \epsilon_{n} \lambda_{n}=\frac{\langle f v, v\rangle}{\|v\|^{2}} \stackrel{\text { def }}{=} \mu .
$$

Finally, for any test function $\phi \in C_{0}^{\infty}(\mathbb{R})$, we have

$$
\begin{aligned}
\epsilon_{n}\left\langle u_{n}, \phi\right\rangle & =\epsilon_{n}\left\langle\left(H_{\epsilon_{n}}-i \lambda_{n}\right) v_{n}, \phi\right\rangle \\
& =\epsilon_{n}\left\langle v_{n}, H_{\infty} \phi\right\rangle+i\left\langle f v_{n}, \phi\right\rangle-i \epsilon_{n} \lambda_{n}\left\langle v_{n}, \phi\right\rangle .
\end{aligned}
$$


Taking the limit $n \rightarrow \infty$, we obtain $\langle f v, \phi\rangle=\mu\langle v, \phi\rangle$ for all $\phi \in C_{0}^{\infty}(\mathbb{R})$, which implies that $(f-\mu) v=0$. Since $f$ and $v$ are continuous functions and $v \not \equiv 0$, this is possible only if $f^{-1}(\mu)$ has non-empty interior. Thus we have shown that, if all level sets of $f$ have empty interior, then necessarily $\Psi(\epsilon) \rightarrow \infty$ as $\epsilon \rightarrow 0$.

Conversely, assume that there exists $\mu \in \mathbb{R}$ such that $f^{-1}(\mu)$ contains a nonempty open interval $I$. Choose $\phi \in C_{0}^{\infty}(I)$ such that $\phi \not \equiv 0$, and let

$$
\psi=H_{\infty} \phi \equiv\left(H_{\epsilon}-\frac{i \mu}{\epsilon}\right) \phi \quad(\text { for any } \epsilon>0) .
$$

Observe that $\psi \not \equiv 0$, because $0 \notin \sigma\left(H_{\infty}\right)$. Thus

$$
\Psi(\epsilon)^{-1} \geq\left\|\left(H_{\epsilon}-\frac{i \mu}{\epsilon}\right)^{-1}\right\| \geq \frac{\|\phi\|}{\|\psi\|}
$$

hence $\Psi(\epsilon) \leq\|\psi\| /\|\phi\|$ for all $\epsilon>0$.

\section{$3 \quad$ Variational estimates}

The purpose of this section is to adapt the general method developed in 24 to our concrete problem, and to show that it allows to obtain precise estimates on the semigroup and the resolvent of the operator $H_{\epsilon}$. To demonstrate the efficiency of this approach, we first give a short proof of Theorem 1.5 in Section 3.1. We next consider in more detail three model problems, and we show how to modify the proof of Theorem 1.5] so as to obtain optimal estimates in each case. With this information at hand, we consider in Section 3.3 the general situation where the function $f$ satisfies Hypothesis 1.6 and we prove the first half of Theorem 1.8, namely the lower bound on $\Psi(\epsilon)$ up to a logarithmic correction. We mention however that a complete proof of Theorem 1.8 will be given in Section 4, using a different method.

\subsection{Proof of Theorem 1.5}

This section is devoted to the proof of Theorem 1.5. Consider any $f \in C^{3}(\mathbb{R})$ such that $f^{\prime \prime}$ and $f^{\prime \prime \prime}$ belong to $L^{\infty}(\mathbb{R})$, and assume that estimate (1.11) holds for some $\nu \in(0,1 / 2]$. Let $u(x, t)$ be a solution to the parabolic equation (1.2), namely

$$
\partial_{t} u(x, t)=\partial_{x}^{2} u(x, t)-x^{2} u(x, t)-\frac{i}{\epsilon} f(x) u(x, t) .
$$

To control the evolution of $u(x, t)$, we introduce the quadratic functional

$$
\Phi(t)=\int_{\mathbb{R}}\left(\frac{1}{2}|u|^{2}+\frac{\alpha}{2}\left(\left|\partial_{x} u\right|^{2}+x^{2}|u|^{2}\right)+\beta \operatorname{Re}\left(\left(\partial_{x} \bar{u}\right) i f^{\prime}(x) u\right)+\frac{\gamma}{2} f^{\prime}(x)^{2}|u|^{2}\right) \mathbf{x},
$$

where $\alpha, \beta, \gamma$ are positive constants to be determined below. We assume that $4 \beta^{2} \leq \alpha \gamma$, so that

$$
\Phi(t) \leq \int_{\mathbb{R}}\left(\frac{1}{2}|u|^{2}+\frac{3 \alpha}{4}\left(\left|\partial_{x} u\right|^{2}+x^{2}|u|^{2}\right)+\frac{3 \gamma}{4} f^{\prime}(x)^{2}|u|^{2}\right) \mathrm{x} .
$$

Note that, according to the general method introduced in [24], the functional $\Phi$ is a linear combination of the quantities $\|u\|^{2},\|A u\|^{2}, \operatorname{Re}\langle A u, C u\rangle$ and $\|C u\|^{2}$, where as in the introduction $A=\partial_{x}+x$ and $C=[A, B]$ with $B=(i / \epsilon) f(x)$. To compute the time derivative of $\Phi(t)$, 
we use the identities:

$$
\begin{aligned}
& \frac{1}{2} \frac{\mathrm{d}}{\mathrm{d} t} \int_{\mathbb{R}}|u|^{2} \mathrm{x}=\operatorname{Re} \int_{\mathbb{R}} \bar{u} \partial_{t} u \mathrm{x}=-\int_{\mathbb{R}}\left(\left|\partial_{x} u\right|^{2}+x^{2}|u|^{2}\right) \mathrm{x}, \\
& \frac{1}{2} \frac{\mathrm{d}}{\mathrm{d} t} \int_{\mathbb{R}} \alpha\left(\left|\partial_{x} u\right|^{2}+x^{2}|u|^{2}\right) \mathrm{x}=-\int_{\mathbb{R}} \alpha\left|\partial_{x}^{2} u-x^{2} u\right|^{2} \mathrm{x}-\operatorname{Re} \int_{\mathbb{R}} \alpha\left(\partial_{x} \bar{u}\right) \frac{i}{\epsilon} f^{\prime}(x) u \mathrm{x}, \\
& \frac{\mathrm{d}}{\mathrm{d} t} \operatorname{Re} \int_{\mathbb{R}} \beta\left(\partial_{x} \bar{u}\right) i f^{\prime}(x) u \mathrm{x}=-\frac{1}{\epsilon} \int_{\mathbb{R}} \beta f^{\prime}(x)^{2}|u|^{2} \mathrm{x}-\operatorname{Re} \int_{\mathbb{R}} \beta \bar{u} i f^{\prime \prime \prime}(x) \partial_{x} u \mathrm{x} \\
& +2 \operatorname{Re} \int_{\mathbb{R}} \beta\left(\partial_{x} \bar{u}\right) i f^{\prime}(x)\left(\partial_{x}^{2} u-x^{2} u\right) \mathbf{x}, \\
& \frac{1}{2} \frac{\mathrm{d}}{\mathrm{d} t} \int_{\mathbb{R}} \gamma f^{\prime}(x)^{2}|u|^{2} \mathrm{x}=-\int_{\mathbb{R}} \gamma f^{\prime}(x)^{2}\left(\left|\partial_{x} u\right|^{2}+x^{2}|u|^{2}\right) \mathrm{x} \\
& -2 \operatorname{Re} \int_{\mathbb{R}} \gamma f^{\prime}(x) f^{\prime \prime}(x) \bar{u} \partial_{x} u x,
\end{aligned}
$$

which follow easily from (3.1) after some integrations by parts. To estimate the various terms in the expression of $\Phi^{\prime}(t)$, we denote

$$
K_{j}=\sup _{x \in \mathbb{R}}\left|\partial_{x}^{j} f(x)\right|, \quad j=2,3,
$$

and we use the following bounds:

$$
\begin{aligned}
& -\alpha \operatorname{Re} \int_{\mathbb{R}}\left(\partial_{x} \bar{u}\right) \frac{i}{\epsilon} f^{\prime}(x) u \mathrm{x} \leq \frac{1}{4} \int_{\mathbb{R}}\left|\partial_{x} u\right|^{2} \mathrm{x}+\frac{\alpha^{2}}{\epsilon^{2}} \int_{\mathbb{R}} f^{\prime}(x)^{2}|u|^{2} \mathrm{x}, \\
& -\beta \operatorname{Re} \int_{\mathbb{R}} \bar{u} i f^{\prime \prime \prime}(x) \partial_{x} u \mathrm{x} \leq K_{3} \beta \int_{\mathbb{R}}\left|u \partial_{x} u\right| \mathrm{x} \leq K_{3} \beta \int_{\mathbb{R}}\left(\left|\partial_{x} u\right|^{2}+x^{2}|u|^{2}\right) \mathrm{x}, \\
& 2 \beta \operatorname{Re} \int_{\mathbb{R}}\left(\partial_{x} \bar{u}\right) i f^{\prime}(x)\left(\partial_{x}^{2} u-x^{2} u\right) \mathrm{x} \leq \frac{\alpha}{2} \int_{\mathbb{R}}\left|\partial_{x}^{2} u-x^{2} u\right|^{2} \mathrm{x}+\frac{2 \beta^{2}}{\alpha} \int_{\mathbb{R}} f^{\prime}(x)^{2}\left|\partial_{x} u\right|^{2} \mathrm{x}, \\
& -2 \gamma \operatorname{Re} \int_{\mathbb{R}} f^{\prime}(x) f^{\prime \prime}(x) \bar{u} \partial_{x} u \mathrm{x} \leq \frac{1}{4} \int_{\mathbb{R}}\left|\partial_{x} u\right|^{2} \mathrm{x}+4 \gamma^{2} K_{2}^{2} \int_{\mathbb{R}} f^{\prime}(x)^{2}|u|^{2} \mathrm{x} .
\end{aligned}
$$

We thus obtain

$$
\begin{aligned}
\Phi^{\prime}(t) & \leq\left(-1+\frac{1}{4}+\frac{1}{4}+K_{3} \beta\right) \int_{\mathbb{R}}\left(\left|\partial_{x} u\right|^{2}+x^{2}|u|^{2}\right) \mathrm{x} \\
& +\left(-\alpha+\frac{\alpha}{2}\right) \int_{\mathbb{R}}\left|\partial_{x}^{2} u-x^{2} u\right|^{2} \mathrm{x}+\left(-\frac{\beta}{\epsilon}+\frac{\alpha^{2}}{\epsilon^{2}}+4 \gamma^{2} K_{2}^{2}\right) \int_{\mathbb{R}} f^{\prime}(x)^{2}|u|^{2} \mathrm{x} \\
& +\left(-\gamma+\frac{2 \beta^{2}}{\alpha}\right) \int_{\mathbb{R}} f^{\prime}(x)^{2}\left(\left|\partial_{x} u\right|^{2}+x^{2}|u|^{2}\right) \mathrm{x} .
\end{aligned}
$$

We now fix

$$
\beta=\min \left(\frac{1}{4 K_{3}}, \frac{1}{32 K_{2}}\right), \quad \alpha=\left(\frac{\beta \epsilon}{4}\right)^{1 / 2}, \quad \gamma=8\left(\frac{\beta^{3}}{\epsilon}\right)^{1 / 2} .
$$

Then $4 \beta^{2} \leq \alpha \gamma, 4 \gamma^{2} K_{2}^{2} \leq \beta /(4 \epsilon)$, and we arrive at the simpler estimate

$$
\begin{aligned}
\Phi^{\prime}(t) \leq & -\frac{1}{4} \int_{\mathbb{R}}\left(\left|\partial_{x} u\right|^{2}+x^{2}|u|^{2}\right) \mathrm{x}-\frac{\beta}{2 \epsilon} \int_{\mathbb{R}} f^{\prime}(x)^{2}|u|^{2} \mathrm{x} \\
& -\frac{\alpha}{2} \int_{\mathbb{R}}\left|\partial_{x}^{2} u-x^{2} u\right|^{2} \mathrm{x}-\frac{\gamma}{2} \int_{\mathbb{R}} f^{\prime}(x)^{2}\left(\left|\partial_{x} u\right|^{2}+x^{2}|u|^{2}\right) \mathrm{x} .
\end{aligned}
$$


In what follows we neglect both terms in (3.15) and keep only the upper bound (3.14). Combining (3.14) with (1.11) and assuming (without loss of generality) that $\epsilon \leq 2 \beta$, we arrive at

$$
\Phi^{\prime}(t) \leq-\frac{1}{8} \int_{\mathbb{R}}\left(\left|\partial_{x} u\right|^{2}+x^{2}|u|^{2}\right) \mathrm{x}-\frac{\beta}{4 \epsilon} \int_{\mathbb{R}} f^{\prime}(x)^{2}|u|^{2} \mathrm{x}-\frac{M_{1}}{8}\left(\frac{2 \beta}{\epsilon}\right)^{\nu} \int_{\mathbb{R}}|u|^{2} \mathrm{x} .
$$

Using finally (3.3), we conclude that $\Phi^{\prime}(t) \leq-\eta \Phi(t)$, where

$$
\eta=\min \left(\frac{1}{6 \alpha}, \frac{\beta}{3 \epsilon \gamma}, \frac{M_{1}}{4}\left(\frac{2 \beta}{\epsilon}\right)^{\nu}\right) .
$$

Since $\nu \leq 1 / 2$ and $\alpha, \beta, \gamma$ are given by (3.13), there exists $M_{2}>0$ such that $\eta \geq 2 M_{2} \epsilon^{-\nu}$ for all $\epsilon \in(0,1]$.

What we have done so far is to construct a quadratic functional $\Phi$ which is equivalent (with $\epsilon$-dependent constants) to the square of the norm in $D\left(H_{\infty}^{1 / 2}\right)=\left\{u \in H^{1}(\mathbb{R}) ; x u \in L^{2}(\mathbb{R})\right\}$, and which satisfies $\Phi(t) \leq e^{-\eta(t-s)} \Phi(s)$ for all $t \geq s \geq 0$. To deduce some information on the semigroup $e^{-t H_{\epsilon}}$ in $L^{2}(\mathbb{R})$, we use the following standard argument. Let $u(x, t)$ be a solution to (3.1) with initial data $u_{0} \in L^{2}(\mathbb{R})$. In view of (3.4), we have

$$
\int_{0}^{\sqrt{\epsilon}}\left(\left\|\partial_{x} u(t)\right\|^{2}+\|x u(t)\|^{2}\right) \mathrm{t}=\frac{1}{2}\left(\left\|u_{0}\right\|^{2}-\|u(t)\|^{2}\right) \leq \frac{1}{2}\left\|u_{0}\right\|^{2},
$$

hence there exists $\tau \in(0, \sqrt{\epsilon}]$ such that

$$
\|u(\tau)\|^{2} \leq\left\|u_{0}\right\|^{2}, \quad\left\|\partial_{x} u(\tau)\right\|^{2}+\|x u(\tau)\|^{2} \leq \frac{\left\|u_{0}\right\|^{2}}{2 \sqrt{\epsilon}} .
$$

Thus, using the definition (3.2) of $\Phi$, the values (3.13) for $\alpha, \beta, \gamma$, and the fact that $\left|f^{\prime}(x)\right| \leq$ $\left|f^{\prime}(0)\right|+K_{2}|x|$ for all $x \in \mathbb{R}$, we see that

$$
\Phi(\sqrt{\epsilon}) \leq \Phi(\tau) \leq \frac{C}{\epsilon}\left\|u_{0}\right\|^{2}, \quad \text { for some } C>0 \text { independent of } \epsilon \in(0,1] .
$$

As a consequence, for any $t \geq 0$, we obtain

$$
\|u(t+\sqrt{\epsilon})\|^{2} \leq 2 \Phi(t+\sqrt{\epsilon}) \leq 2 e^{-\eta t} \Phi(\sqrt{\epsilon}) \leq \frac{2 C}{\epsilon} e^{-\eta t}\left\|u_{0}\right\|^{2},
$$

hence

$$
\left\|e^{-(t+\sqrt{\epsilon}) H_{\epsilon}}\right\| \leq\left(\frac{2 C}{\epsilon}\right)^{1 / 2} e^{-M_{2} t / \epsilon^{\nu}}, \quad \text { for all } t \geq 0 .
$$

Since we also have $\left\|e^{-t H_{\epsilon}}\right\| \leq 1$ for all $t \geq 0$, it follows from Lemma 1.17i) that $\Sigma(\epsilon) \geq M_{2} / \epsilon^{\nu}$. Moreover, proceeding as in the proof of that lemma (see Appendix $\mathrm{A}$ ), we find

$$
\begin{aligned}
\Psi(\epsilon)^{-1} & \leq \int_{0}^{\infty}\left\|e^{-t H_{\epsilon}}\right\| \mathrm{t} \leq \int_{0}^{\sqrt{\epsilon}} \mathrm{t}+\int_{0}^{\infty} \min \left\{1,\left(\frac{2 C}{\epsilon}\right)^{1 / 2} e^{-M_{2} t / \epsilon^{\nu}}\right\} \mathrm{t} \\
& \leq \sqrt{\epsilon}+\frac{\epsilon^{\nu}}{M_{2}}\left(1+\log \left(\frac{2 C}{\epsilon}\right)^{1 / 2}\right) .
\end{aligned}
$$

Since $\nu \leq 1 / 2$, we deduce that $\Psi(\epsilon)^{-1} \leq C^{\prime} \epsilon^{\nu} \log (2 / \epsilon)$. This concludes the proof of Theorem 1.5. 


\subsection{Three model problems}

A simple and general result such as Theorem 1.5 cannot give optimal estimates for all possible choices of the function $f$. In particular, if $f$ satisfies Hypothesis 1.6, the lower bounds (1.12) with $\nu=(k+2)^{-1}$ (see Lemma 1.7) are too pessimistic. In this paragraph we consider three representative examples, and we show in each case that the proof of Theorem 1.5 gives in fact an optimal lower bound on $\Psi(\epsilon)$ provided we choose in an appropriate way the parameters $\alpha, \beta, \gamma$ entering in the definition (3.2) of the functional $\Phi$. These examples are the building blocks which will allow us to treat in Section 3.3 the general case of a function $f$ satisfying Hypothesis 1.6. It is interesting to note that the proof of Theorem 1.8 in Section 4 involves a similar enumeration of cases, see Proposition 4.1

\subsubsection{The case of a nondegenerate critical point}

We first consider the situation where $f$ has a unique, non nondegenerate critical point, and $\left|f^{\prime}\right|$ is bounded away from zero outside a neighborhood of this point. The simplest example of such a function is $f(x)=x^{2}$. In that case we can just follow the proof of Theorem 1.5 and choose the parameters $\alpha, \beta, \gamma$ as in (3.13). Indeed, the lower bound (1.11) holds with $\nu=1 / 2$, hence it follows from (3.16) that $\Phi^{\prime}(t) \leq-\eta \Phi(t)$ with $\eta=\mathcal{O}\left(\epsilon^{-1 / 2}\right)$. This result is clearly optimal, because we know that $\Sigma(\epsilon)=\operatorname{Re}\left((1+i / \epsilon)^{1 / 2}\right)=\mathcal{O}\left(\epsilon^{-1 / 2}\right)$ if $f(x)=x^{2}$.

\subsubsection{The case when $f$ has no critical point}

We next study the case when $\left|f^{\prime}\right|$ is bounded away from zero. The simplest paradigm here is $f(x)=x$. In this example, we have

$$
H_{\epsilon}=-\partial_{x}^{2}+x^{2}+\frac{i}{\epsilon} x=-\partial_{x}^{2}+\left(x+\frac{i}{2 \epsilon}\right)^{2}+\frac{1}{4 \epsilon^{2}},
$$

hence $\Sigma(\epsilon)=1+\left(4 \epsilon^{2}\right)^{-1}$. One can also estimate the resolvent of $H_{\epsilon}$, and one finds that $\Psi(\epsilon)=\mathcal{O}\left(\epsilon^{-2 / 3}\right)$, see Proposition 4.1. To recover this result using the variational method, it is necessary to modify slightly the proof of Theorem 1.5. Indeed, if we still choose $\alpha, \beta, \gamma$ as in (3.13), then (3.16) again implies $\eta=\mathcal{O}\left(\epsilon^{-1 / 2}\right)$, because the lower bound (1.11) holds with $\nu=1$. However, since $f^{\prime} \equiv 1$, it is more natural here to set $\gamma=0$ in (3.2) and to assume that $\beta^{2} \leq \alpha / 4$, so that $\Phi(t)$ is strictly positive. Then proceeding exactly as above and using the fact that the terms (3.7), (3.9), and (3.11) vanish identically, we see that (3.12) becomes

$$
\begin{aligned}
\Phi^{\prime}(t) & \leq\left(-1+\frac{1}{4}+\frac{2 \beta^{2}}{\alpha}\right) \int_{\mathbb{R}}\left(\left|\partial_{x} u\right|^{2}+x^{2}|u|^{2}\right) \mathrm{x} \\
& +\left(-\alpha+\frac{\alpha}{2}\right) \int_{\mathbb{R}}\left|\partial_{x}^{2} u-x^{2} u\right|^{2} \mathrm{x}+\left(-\frac{\beta}{\epsilon}+\frac{\alpha^{2}}{\epsilon^{2}}\right) \int_{\mathbb{R}} f^{\prime}(x)^{2}|u|^{2} \mathrm{x} .
\end{aligned}
$$

We need $\alpha^{2} \leq \epsilon \beta / 2$, so we can choose for instance

$$
\alpha=\left(\frac{\epsilon^{2}}{16}\right)^{\frac{1}{3}}, \text { and } \beta=\left(\frac{\epsilon}{32}\right)^{\frac{1}{3}} .
$$

Then we easily obtain $\Phi^{\prime}(t) \leq-\eta \Phi(t)$ with

$$
\eta=\min \left(\frac{1}{3 \alpha}, \frac{2 \beta}{3 \epsilon}\right)=\mathcal{O}\left(\epsilon^{-\frac{2}{3}}\right) .
$$

Remark: In the more general case when $f^{\prime}$ is not identically constant, but still bounded away from zero, we have to deal with the additional term which appears in the left-hand side of (3.9). Since $\left|f^{\prime \prime \prime}\right| \leq C\left|f^{\prime}\right|$, this term is easily bounded as in (3.8), with $\alpha / \epsilon$ replaced by $\beta$. 


\subsubsection{The case of a critical point at infinity}

Finally, we consider the situation where $f$ has no critical point, but $f^{\prime}(x)$ decreases algebraically as $x \rightarrow \pm \infty$. We fix $k>0$ and focus on the following example:

$$
f(x)=\int_{0}^{x} \frac{y}{\left(1+y^{2}\right)^{\frac{k+1}{2}}}, \quad x \in \mathbb{R},
$$

which nearly satisfies Hypothesis [1.6. In particular, $f^{\prime}(x)=\mathcal{O}\left(|x|^{-k-1}\right)$ as $|x| \rightarrow \infty$. The proof of Theorem 1.8 given in Section 4 applies to this example, and shows that $\Psi(\epsilon)=\mathcal{O}\left(\epsilon^{-\bar{\nu}}\right)$ where $\bar{\nu}=2 /(k+4)$. On the other hand, the lower bound (1.11) holds with $\nu=(k+2)^{-1}<\bar{\nu}$, hence the proof of Theorem 1.5 only gives $\Phi^{\prime}(t) \leq-\eta \Phi(t)$ with $\eta=\mathcal{O}\left(\epsilon^{-\nu}\right)$. However, we shall show how to choose the parameters $\alpha, \beta, \gamma$ in (3.2) to obtain the optimal result in this case too.

So let us again consider the functional $\Phi(t)$, supposing as usual $\beta^{2} \leq \alpha \gamma / 4$ to ensure (3.3), and let us go through estimates (3.8) to (3.11). The main advantage we have here compared to the general situation considered in Theorem 1.5 is that $\left|f^{\prime \prime}\right|+\left|f^{\prime \prime \prime}\right| \leq C_{k}\left|f^{\prime}\right|$, where $C_{k}$ denotes (here and below) a generic constant depending only on $k$. Estimates (3.8) and (3.10), which involve only $f^{\prime}$, are unchanged. Estimate (3.9) is replaced by

$$
-\beta \operatorname{Re} \int_{\mathbb{R}} \bar{u} i f^{\prime \prime \prime}(x) \partial_{x} u \mathrm{x} \leq \frac{1}{4} \int_{\mathbb{R}}\left|\partial_{x} u\right|^{2} \mathrm{x}+C_{k} \beta^{2} \int_{\mathbb{R}} f^{\prime}(x)^{2}|u|^{2} \mathrm{x} .
$$

Finally, after integrating by parts, we bound the left-hand side of (3.11) as follows:

$$
-2 \gamma \operatorname{Re} \int_{\mathbb{R}} f^{\prime}(x) f^{\prime \prime}(x) \bar{u} \partial_{x} u \underline{x}=\gamma \int_{\mathbb{R}}\left(f^{\prime \prime}(x)^{2}+f^{\prime}(x) f^{\prime \prime \prime}(x)\right)|u|^{2} \mathrm{x} \leq C_{k} \gamma \int_{\mathbb{R}} f^{\prime}(x)^{2}|u|^{2} \mathrm{x} .
$$

Summarizing, estimate (3.12) becomes

$$
\begin{aligned}
\Phi^{\prime}(t) \leq & -\frac{1}{2} \int_{\mathbb{R}}\left(\left|\partial_{x} u\right|^{2}+x^{2}|u|^{2}\right) \mathrm{x}+\left(-\frac{\beta}{\epsilon}+\frac{\alpha^{2}}{\epsilon^{2}}+C_{k}\left(\beta^{2}+\gamma\right)\right) \int_{\mathbb{R}} f^{\prime}(x)^{2}|u|^{2} \mathrm{x} \\
& -\frac{\alpha}{2} \int_{\mathbb{R}}\left|\partial_{x}^{2} u-x^{2} u\right|^{2} \mathrm{x}+\left(-\gamma+\frac{2 \beta^{2}}{\alpha}\right) \int_{\mathbb{R}} f^{\prime}(x)^{2}\left(\left|\partial_{x} u\right|^{2}+x^{2}|u|^{2}\right) \mathrm{x} .
\end{aligned}
$$

We now assume that $\alpha^{2} \leq \beta \epsilon / 4$ and $C_{k}\left(\beta^{2}+\gamma\right) \leq \beta /(4 \epsilon)$. Using in addition the lower bound (1.11), which holds for the value $\nu=(k+2)^{-1}$ in view of Lemma 1.7, we obtain

$$
\Phi^{\prime}(t) \leq-\frac{1}{4} \int_{\mathbb{R}}\left(\left|\partial_{x} u\right|^{2}+x^{2}|u|^{2}\right) \mathrm{x}-\frac{\beta}{4 \epsilon} \int_{\mathbb{R}} f^{\prime}(x)^{2}|u|^{2} \mathrm{x}-\frac{M_{1}}{4}\left(\frac{\beta}{\epsilon}\right)^{\nu} \int_{\mathbb{R}}|u|^{2} \mathrm{x} .
$$

It follows that $\Phi^{\prime}(t) \leq-\eta \Phi(t)$ with

$$
\eta=\min \left(\frac{1}{3 \alpha}, \frac{\beta}{3 \epsilon \gamma}, \frac{M_{1}}{2}\left(\frac{\beta}{\epsilon}\right)^{\nu}\right) .
$$

If we now choose $\alpha=\frac{1}{2} \epsilon^{\frac{2}{k+4}}, \beta=\epsilon^{-\frac{k}{k+4}}$, and $\gamma=8 \epsilon^{-\frac{2 k+2}{k+4}}$, we obtain $\eta=\mathcal{O}\left(\epsilon^{-\frac{2}{k+4}}\right)$ which is the desired result.

\subsection{Improved decay rate under Hypothesis 1.6}

Let us now consider an arbitrary function $f$ satisfying Hypothesis 1.6. The analysis of Section 3.2 suggests considering three different regions of space (near the critical points of $f$, near infinity, 
or away from those regions), and shows how to choose the parameters $\alpha, \beta, \gamma$ so that the functional $\Phi$ defined by (3.2) decays with the optimal rate in each region. Since different choices are needed in different regions, it is very natural to generalize the definition (3.2) and to allow the parameters $\alpha, \beta, \gamma$ to depend on the space variable $x$. So we consider again the functional

$$
\Phi(t)=\int_{\mathbb{R}}\left(\frac{1}{2}|u|^{2}+\frac{\alpha}{2}\left(\left|\partial_{x} u\right|^{2}+x^{2}|u|^{2}\right)+\beta \operatorname{Re}\left(\left(\partial_{x} \bar{u}\right) i f^{\prime}(x) u\right)+\frac{\gamma}{2} f^{\prime}(x)^{2}|u|^{2}\right) \mathrm{x},
$$

where now $\alpha, \beta, \gamma$ are positive functions of $x$, depending also on $\epsilon$. To choose these functions in the simplest possible way, we first decide that

$$
\alpha=\left(\frac{\beta \epsilon}{4}\right)^{\frac{1}{2}}, \quad \text { and } \quad \gamma=8\left(\frac{\beta^{3}}{\epsilon}\right)^{\frac{1}{2}} \text {. }
$$

Indeed this relation between $\alpha, \gamma$ and $\beta, \epsilon$ was already used in (3.13), so if we impose (3.17) our set of parameters will be convenient near the critical points of $f$, provided $\beta$ is small enough. Moreover, as is easily verified, the parameters we chose in Section 3.2.3 also satisfy (3.17), so this definition will be adapted near infinity provided $\beta$ is of the order of $\epsilon^{-k /(k+4)}$ in that region. Finally, in the intermediate region we can choose the same parameters $\alpha, \beta, \gamma$ as near the critical points of $f$, because (as was observed in Section 3.2.2) the functional $\Phi$ will decay with the suboptimal rate $\eta=\mathcal{O}\left(\epsilon^{-1 / 2}\right)$ which is still better than what we have near infinity. Summarizing, the choice (3.17) is appropriate in all regions provided $\beta$ is small near the critical points of $f$ and $\beta=\mathcal{O}\left(\epsilon^{-k /(k+4)}\right)$ near infinity. Note that (3.17) implies $\alpha \gamma=4 \beta^{2}$, so that the upper bound (3.3) is still valid.

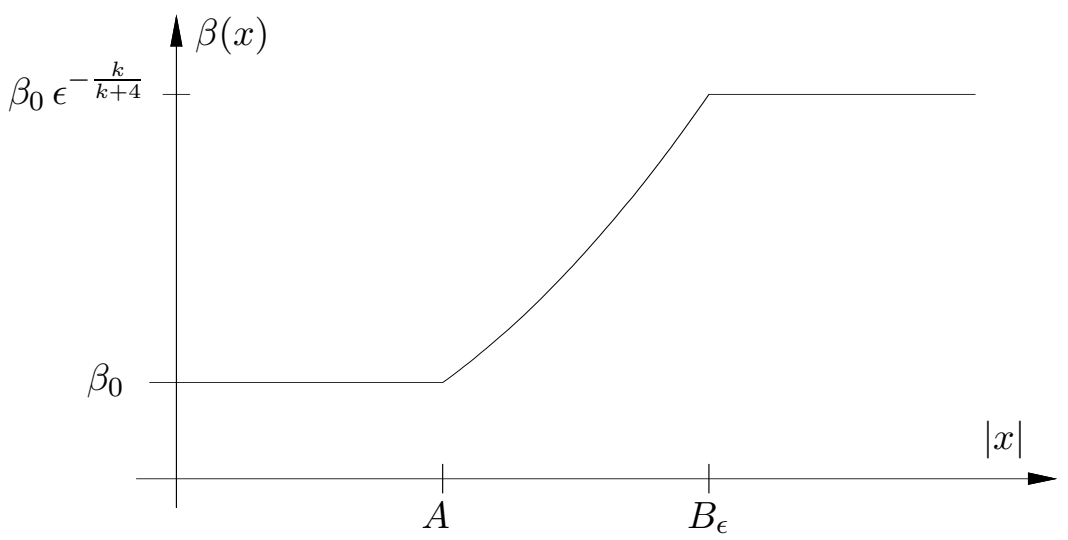

Fig. 1: The graph of the function $\beta$ defined in (3.18). Here $B_{\epsilon}=A \epsilon^{-\frac{1}{2(k+4)}}$, and $A>0$ is sufficiently large so that all critical points of $f$ are contained in the interval $[-A+1, A-1]$.

It remains to choose the function $\beta$ appropriately. Let $\beta_{0}>0$ be a small constant, and $A>0$ be large enough so that that all critical points of $f$ are contained in the interval $[-A+1, A-1]$. Both parameters are independent of $\epsilon$, and their precise values will be specified later. We define the function $\beta: \mathbb{R} \rightarrow \mathbb{R}_{+}$by

$$
\beta(x)=\left\{\begin{array}{lll}
\beta_{0} & \text { if } & |x| \leq A \\
\beta_{0}\left(\frac{|x|}{A}\right)^{2 k} & \text { if } & |A| \leq|x| \leq B_{\epsilon} \\
\beta_{0} \epsilon^{-\frac{k}{k+4}} & \text { if } & |x| \geq B_{\epsilon}
\end{array}\right.
$$


where $k>0$ is as in Hypothesis 1.6 and $B_{\epsilon}=A \epsilon^{-\frac{1}{2(k+4)}}$. The graph of $\beta$ is represented in Fig. 1 . It is easily verified that $\beta$ has the following useful properties: for any $c>0$, there exists $\epsilon_{0}>0$ such that, for any $\epsilon \in\left(0, \epsilon_{0}\right)$ and all $x \in \mathbb{R}$,

$$
\epsilon^{\frac{1}{2}} \beta^{\prime}(x)^{2} \leq c \beta(x)^{\frac{3}{2}}, \quad \epsilon \beta(x) \leq c, \quad \text { and } \quad \epsilon \beta^{\prime}(x)^{2} \leq c \beta(x) .
$$

We now compute the time derivative of $\Phi(t)$, taking into account the additional terms involving derivatives of $\alpha, \beta, \gamma$ which result from integrations by parts. We do not give the full details here, but simply indicate the main modifications with respect to the corresponding calculations in Section 3.1. Equation (3.4) is of course unchanged, so we start with (3.5). Since $\alpha$ now depends on $x$, the right-hand side of (3.5) contains an additional term which can be bounded as follows:

$$
-\operatorname{Re} \int_{\mathbb{R}} \alpha^{\prime}\left(\partial_{x} \bar{u}\right)\left(\partial_{x}^{2} u-x^{2} u\right) \mathrm{x} \leq \frac{1}{4} \int_{\mathbb{R}} \alpha\left|\partial_{x}^{2} u-x^{2} u\right|^{2} \mathrm{x}+\int_{\mathbb{R}} \frac{\alpha^{\prime 2}}{\alpha}\left|\partial_{x} u\right|^{2} \mathrm{x} .
$$

Remark that, in view of (3.19), we have for $\epsilon>0$ small enough:

$$
\frac{\alpha^{\prime}(x)^{2}}{\alpha(x)}=\frac{\epsilon^{1 / 2} \beta^{\prime}(x)^{2}}{8 \beta(x)^{3 / 2}} \leq \frac{1}{12}, \text { for all } x \in \mathbb{R} .
$$

On the other hand, since $\alpha^{2}=\beta \epsilon / 4$, we have by (3.8)

$$
-\operatorname{Re} \int_{\mathbb{R}} \alpha\left(\partial_{x} \bar{u}\right) \frac{i}{\epsilon} f^{\prime}(x) u \mathrm{x} \leq \frac{1}{4} \int_{\mathbb{R}}\left|\partial_{x} u\right|^{2} \mathrm{x}+\frac{1}{4 \epsilon} \int_{\mathbb{R}} \beta f^{\prime}(x)^{2}|u|^{2} \mathrm{x} .
$$

We conclude that

$$
\begin{aligned}
\frac{1}{2} \frac{\mathrm{d}}{\mathrm{d} t} \int_{\mathbb{R}} \alpha\left(\left|\partial_{x} u\right|^{2}+x^{2}|u|^{2}\right) \mathrm{x} \leq & -\int_{\mathbb{R}} \frac{3 \alpha}{4}\left|\partial_{x}^{2} u-x^{2} u\right|^{2} \mathrm{x} \\
& +\frac{1}{3} \int_{\mathbb{R}}\left|\partial_{x} u\right|^{2} \mathrm{x}+\int_{\mathbb{R}} \frac{\beta}{4 \epsilon} f^{\prime}(x)^{2}|u|^{2} \mathbf{x} .
\end{aligned}
$$

We next consider (3.6). In that case one should add to the right-hand side the terms

$$
\operatorname{Re} \int_{\mathbb{R}} \beta^{\prime} \bar{u} i f^{\prime}(x)\left(\partial_{x}^{2} u-x^{2} u\right) \mathrm{x}-\operatorname{Re} \int_{\mathbb{R}} \beta^{\prime} \bar{u} i f^{\prime \prime}(x) \partial_{x} u \mathrm{x} .
$$

The first one is easily controlled, for $\epsilon$ small enough, by

$$
\begin{aligned}
\operatorname{Re} \int_{\mathbb{R}} \beta^{\prime} \bar{u} i f^{\prime}(x)\left(\partial_{x}^{2} u-x^{2} u\right) \mathrm{x} & \leq \int_{\mathbb{R}} \frac{\alpha}{8}\left|\partial_{x}^{2} u-x^{2} u\right|^{2} \mathrm{x}+\int_{\mathbb{R}} \frac{2 \beta^{\prime 2}}{\alpha} f^{\prime}(x)^{2}|u|^{2} \mathrm{x} \\
& \leq \int_{\mathbb{R}} \frac{\alpha}{8}\left|\partial_{x}^{2} u-x^{2} u\right|^{2} \mathbf{x}+\int_{\mathbb{R}} \frac{\beta}{12 \epsilon} f^{\prime}(x)^{2}|u|^{2} \mathbf{x},
\end{aligned}
$$

because $\beta^{\prime 2} / \alpha \ll \beta / \epsilon$ due to (3.17), (3.19). Similarly, the second term is estimated by

$$
\begin{aligned}
-\operatorname{Re} \int_{\mathbb{R}} \beta^{\prime} \bar{u} i f^{\prime \prime}(x) \partial_{x} u \mathrm{x} & \leq \frac{1}{12} \int_{\mathbb{R}}\left|\partial_{x} u\right|^{2} \mathrm{x}+\int_{\mathbb{R}} 3 \beta^{\prime 2} f^{\prime \prime}(x)^{2}|u|^{2} \mathrm{x} \\
& \leq \frac{1}{12} \int_{\mathbb{R}}\left|\partial_{x} u\right|^{2} \mathbf{x}+\int_{\mathbb{R}} \frac{\beta}{12 \epsilon} f^{\prime}(x)^{2}|u|^{2} \mathbf{x},
\end{aligned}
$$


where in the last inequality we have used (3.19), as well as the fact that $\left|f^{\prime \prime}(x)\right| \leq C_{k}\left|f^{\prime}(x)\right|$ for all $x$ in the support of $\beta^{\prime}$. On the other hand, we have as in (3.10):

$$
2 \operatorname{Re} \int_{\mathbb{R}} \beta\left(\partial_{x} \bar{u}\right) i f^{\prime}(x)\left(\partial_{x}^{2} u-x^{2} u\right) \underline{\mathrm{x}} \leq \int_{\mathbb{R}} \frac{\alpha}{2}\left|\partial_{x}^{2} u-x^{2} u\right|^{2} \mathbf{x}+\int \frac{\gamma}{2} f^{\prime}(x)^{2}\left|\partial_{x} u\right|^{2} \mathbf{x},
$$

where we have used the fact that $4 \beta^{2}=\alpha \gamma$. Finally, the term involving $f^{\prime \prime \prime}(x)$ in (3.5) is estimated as follows. We first note that

$$
-\operatorname{Re} \int_{\mathbb{R}} \beta \bar{u} i f^{\prime \prime \prime} \partial_{x} u \mathrm{x} \leq \frac{1}{12} \int_{\mathbb{R}}\left|\partial_{x} u\right|^{2} \mathrm{x}+3 \int_{|x| \leq A} \beta^{2} f^{\prime \prime \prime 2}|u|^{2} \mathrm{x}+3 \int_{|x| \geq A} \beta^{2} f^{\prime \prime \prime 2}|u|^{2} \mathrm{x} .
$$

In the region $|x| \leq A$, we have

$$
3 \int_{|x| \leq A} \beta^{2} f^{\prime \prime \prime}(x)^{2}|u|^{2} \mathbf{x} \leq 3 \beta_{0}^{2} K_{3}^{2} \int_{\mathbb{R}}|u|^{2} \mathbf{x} \leq 3 \beta_{0}^{2} K_{3}^{2} \int_{\mathbb{R}}\left(\left|\partial_{x} u\right|^{2}+x^{2}|u|^{2}\right) \mathbf{x},
$$

and we choose $\beta_{0}>0$ small enough so that $3 \beta_{0}^{2} K_{3}^{2} \leq 1 / 6$. In the region $|x| \geq A$ we know that $\left|f^{\prime \prime \prime}(x)\right| \leq C_{k}\left|f^{\prime}(x)\right|$, and in view of (3.19) we find for $\epsilon$ small enough:

$$
3 \int_{|x| \geq A} \beta^{2} f^{\prime \prime \prime}(x)^{2}|u|^{2} \mathbf{x} \leq \int_{\mathbb{R}} \frac{\beta}{12 \epsilon} f^{\prime}(x)^{2}|u|^{2} \mathbf{x} .
$$

Summarizing, we have shown that

$$
\begin{aligned}
\frac{\mathrm{d}}{\mathrm{d} t} \operatorname{Re} \int_{\mathbb{R}} \beta\left(\partial_{x} \bar{u}\right) i f^{\prime}(x) u \mathrm{x} \leq & -\int_{\mathbb{R}} \frac{3 \beta}{4 \epsilon} f^{\prime}(x)^{2}|u|^{2} \mathbf{x}+\frac{1}{3} \int_{\mathbb{R}}\left(\left|\partial_{x} u\right|^{2}+x^{2}|u|^{2}\right) \mathbf{x} \\
& +\int_{\mathbb{R}} \frac{5 \alpha}{8}\left|\partial_{x}^{2} u-x^{2} u\right|^{2} \mathbf{x}+\int_{\mathbb{R}} \frac{\gamma}{2} f^{\prime}(x)^{2}\left|\partial_{x} u\right|^{2} \mathbf{x} .
\end{aligned}
$$

Finally we turn our attention to (3.7). This time the right-hand side contains only one additional term, which can be estimated as follows:

$$
\begin{aligned}
-\operatorname{Re} \int_{\mathbb{R}} \gamma^{\prime} f^{\prime}(x)^{2} \bar{u} \partial_{x} u \mathrm{x} & \leq \int_{\mathbb{R}} \frac{\gamma}{4} f^{\prime}(x)^{2}\left|\partial_{x} u\right|^{2} \mathrm{x}+\int_{\mathbb{R}} \frac{\gamma^{\prime 2}}{\gamma} f^{\prime}(x)^{2}|u|^{2} \mathrm{x} \\
& \leq \int_{\mathbb{R}} \frac{\gamma}{4} f^{\prime}(x)^{2}\left|\partial_{x} u\right|^{2} \mathrm{x}+\int_{\mathbb{R}} \frac{\beta}{12 \epsilon} f^{\prime}(x)^{2}|u|^{2} \mathrm{x},
\end{aligned}
$$

where we have used the fact that $\gamma^{\prime 2} / \gamma \ll \beta / \epsilon$ if $\epsilon$ is small, see (3.17), (3.19). It remains to bound the last term in the right-hand side of (3.7). Using a standard ( $\epsilon$-independent) partition of unity and we decompose $\gamma$ as $\gamma=\gamma_{1}+\gamma_{2}$, where $\gamma_{1}$ and $\gamma_{2}$ are nonnegative Lipschitz functions satisfying $\operatorname{supp}\left(\gamma_{1}\right) \subset\{|x| \leq 2 A\}$ and $\operatorname{supp}\left(\gamma_{2}\right) \subset\{|x| \geq 3 A / 2\}$. Then we observe that

$$
\begin{aligned}
-2 \operatorname{Re} \int_{\mathbb{R}} \gamma_{1} f^{\prime}(x) f^{\prime \prime}(x) \bar{u} \partial_{x} u \mathrm{x} & \leq \int_{\mathbb{R}} \frac{\beta}{12 \epsilon} f^{\prime}(x)^{2}|u|^{2} \mathrm{X}+12 K_{2}^{2} \int_{|x| \leq 2 A} \frac{\epsilon \gamma^{2}}{\beta}\left|\partial_{x} u\right|^{2} \mathrm{x} \\
& \leq \int_{\mathbb{R}} \frac{\beta}{12 \epsilon} f^{\prime}(x)^{2}|u|^{2} \mathrm{X}+\frac{1}{12} \int_{\mathbb{R}}\left|\partial_{x} u\right|^{2} \mathrm{x},
\end{aligned}
$$

where in the second inequality we have used the relation $\epsilon \gamma^{2} / \beta=64 \beta^{2}$ and the fact that $\beta(x) \leq \beta_{0} 2^{2 k}$ if $|x| \leq 2 A$. We have also chosen $\beta_{0}>0$ small enough so that $768 \beta_{0}^{2} K_{2}^{2} 2^{4 k} \leq 1 / 12$. On the other hand, integrating by parts, we find

$$
-2 \operatorname{Re} \int_{\mathbb{R}} \gamma_{2} f^{\prime}(x) f^{\prime \prime}(x) \bar{u} \partial_{x} u \underline{x}=\int_{\mathbb{R}} \gamma_{2}\left(f^{\prime \prime 2}+f^{\prime} f^{\prime \prime \prime}\right)|u|^{2} \mathbf{x}+\int_{\mathbb{R}} \gamma_{2}^{\prime} f^{\prime} f^{\prime \prime}|u|^{2} \mathrm{x} .
$$


We know that $\left|f^{\prime \prime}\right|+\left|f^{\prime \prime \prime}\right| \leq C_{k}\left|f^{\prime}\right|$ on the support of $\gamma_{2}$. Moreover, since $\left|\gamma^{\prime} / \gamma\right| \leq \frac{3}{2}\left|\beta^{\prime} / \beta\right| \leq C$ by (3.18), it is clear that $\left|\gamma_{2}^{\prime}\right| \leq C \gamma$ for some $C>0$ independent of $\epsilon$. Thus

$$
-2 \operatorname{Re} \int_{\mathbb{R}} \gamma_{2} f^{\prime}(x) f^{\prime \prime}(x) \bar{u} \partial_{x} u \mathrm{x} \leq C \int_{\mathbb{R}} \gamma f^{\prime}(x)^{2}|u|^{2} \mathrm{x} \leq \int_{\mathbb{R}} \frac{\beta}{12 \epsilon} f^{\prime}(x)^{2}|u|^{2} \mathrm{x},
$$

because $\gamma \ll \beta / \epsilon$ if $\epsilon$ is small, see (3.17), (3.19). We conclude that

$$
\begin{aligned}
\frac{1}{2} \frac{\mathrm{d}}{\mathrm{d} t} \int_{\mathbb{R}} \gamma f^{\prime 2}|u|^{2} \mathrm{x} \leq & -\int_{\mathbb{R}} \frac{3 \gamma}{4} f^{\prime}(x)^{2}\left(\left|\partial_{x} u\right|^{2}+x^{2}|u|^{2}\right) \mathrm{x} \\
& +\frac{1}{12} \int_{\mathbb{R}}\left|\partial_{x} u\right|^{2} \mathrm{x}+\int_{\mathbb{R}} \frac{\beta}{4 \epsilon} f^{\prime}(x)^{2}|u|^{2} \mathbf{x} .
\end{aligned}
$$

Summarizing, if we collect estimates (3.4), (3.20), (3.21), and (3.22), we obtain

$$
\begin{aligned}
\Phi^{\prime}(t) \leq & -\frac{1}{4} \int_{\mathbb{R}}\left(\left|\partial_{x} u\right|^{2}+x^{2}|u|^{2}\right) \mathrm{x}-\int_{\mathbb{R}} \frac{\beta}{4 \epsilon} f^{\prime}(x)^{2}|u|^{2} \mathrm{x} \\
& -\int_{\mathbb{R}} \frac{\alpha}{8}\left|\partial_{x}^{2} u-x^{2} u\right|^{2} \mathrm{x}-\int_{\mathbb{R}} \frac{\gamma}{4} f^{\prime}(x)^{2}\left(\left|\partial_{x} u\right|^{2}+x^{2}|u|^{2}\right) \mathrm{x},
\end{aligned}
$$

and as in Section 3.1 it is sufficient to keep only the first line in (3.23). On the other hand, with our choice of the function $\beta$, it is proved in Lemma A.1 that there exists $M_{0}>0$ such that

$$
\int_{\mathbb{R}}\left(\left|\partial_{x} u\right|^{2}+x^{2}|u|^{2}+\frac{\beta}{\epsilon} f^{\prime}(x)^{2}|u|^{2}\right) \mathrm{x} \geq \frac{M_{0}}{\epsilon^{\bar{\nu}}}\|u\|_{L^{2}}^{2}, \quad \text { where } \quad \bar{\nu}=\frac{2}{k+4} .
$$

Combining (3.24) with the first line of (3.23), we easily obtain

$$
\Phi^{\prime}(t) \leq-\frac{1}{8} \int_{\mathbb{R}}\left(\left|\partial_{x} u\right|^{2}+x^{2}|u|^{2}\right) \mathrm{x}-\int_{\mathbb{R}} \frac{\beta}{8 \epsilon} f^{\prime}(x)^{2}|u|^{2} \mathrm{x}-\frac{M_{0}}{8 \epsilon^{\bar{\nu}}} \int_{\mathbb{R}}|u|^{2} \mathrm{x} .
$$

Using now the upper bound (3.3), we deduce that $\Phi^{\prime}(t) \leq-\eta \Phi(t)$ if

$$
\eta=\min \left(\frac{1}{6\|\alpha\|_{L^{\infty}}}, \frac{1}{6 \epsilon}\left\|\frac{\gamma}{\beta}\right\|_{L^{\infty}}^{-1}, \frac{M_{0}}{8 \epsilon^{\bar{\nu}}}\right)=\mathcal{O}\left(\epsilon^{-\bar{\nu}}\right) .
$$

As in Section 3.1, we conclude that $\Psi(\epsilon)^{-1} \leq C \epsilon^{\bar{\nu}} \log (2 / \epsilon)$ for some $C>0$ independent of $\epsilon$. Up to a logarithmic correction, this proves the lower bound in (1.15) with the rate $\epsilon^{\bar{\nu}}$, which is better than $\epsilon^{\nu}$ since $\nu=(k+2)^{-1}<\bar{\nu}$.

\section{Resolvent estimates}

In this section we obtain precise estimates on the resolvent of $H_{\epsilon}$ along the imaginary axis, and thereby prove Theorem 1.8. In doing so, we construct a nontrivial complex domain $\omega_{\epsilon}$, parametrized by $\epsilon \in(0,1]$, which avoids the pseudospectrum of $H_{\epsilon}$ as $\epsilon \rightarrow 0$ in the sense of Definition 1.2. The boundary of this domain is a graph over the imaginary axis which exhibits a complicated structure involving various $\epsilon$-dependent scales, see Fig. 2 below. This phenomenon does not occur in familiar examples such as self-adjoint operators, kinetic FokkerPlanck operators, or complex harmonic oscillators, see [4]. Our proof will show that the value of the quantity $\Psi(\epsilon)$ results from the competition between various microlocal models related either to the critical points of $f$ or to the behavior of $f$ at infinity. 
Throughout this section, we assume that $f: \mathbb{R} \rightarrow \mathbb{R}$ satisfies Hypothesis 1.6. In particular $\overline{f(\mathbb{R})}=f(\mathbb{R}) \cup\{0\}$ and $f$ has only a finite number of critical points. The finite set of critical values of $f$ is denoted by

$$
\operatorname{cv}(f)=\left\{f(x) ; x \in \mathbb{R}, f^{\prime}(x)=0\right\} .
$$

For any $\lambda \in \mathbb{R}$ and any $\epsilon \in(0,1)$, we define

$$
\kappa(\epsilon, \lambda)=\left\|\left(H_{\epsilon}-i \lambda\right)^{-1}\right\| .
$$

The following proposition gives accurate bounds on $\kappa(\epsilon, \lambda)$ in various parameter regimes:

Proposition 4.1 For $\epsilon \in(0,1)$ and $\lambda \in \mathbb{R}$, the quantity $\kappa(\epsilon, \lambda)$ defined in (4.1) satisfies the following estimates:

i) If $\operatorname{dist}(\epsilon \lambda, f(\mathbb{R})) \geq \delta>0$, then $\kappa(\epsilon, \lambda) \leq \epsilon / \delta$.

ii) If $\operatorname{dist}(\epsilon \lambda, \operatorname{cv}(f) \cup\{0\}) \geq \delta>0$, then $\kappa(\epsilon, \lambda) \leq C_{\delta} \epsilon^{2 / 3}$.

iii) If $\lambda=\lambda(\epsilon)$ is such that $\lim _{\epsilon \rightarrow 0} \epsilon \lambda(\epsilon)=c \in \operatorname{cv}(f) \backslash\{0\}$, then $\lim _{\sup _{\epsilon \rightarrow 0}} \epsilon^{-1 / 2} \kappa(\epsilon, \lambda(\epsilon)) \leq C$.

iv) For $\lambda=0$, the quantity $\kappa(\epsilon, 0)$ satisfies

$$
\kappa(\epsilon, 0) \leq \begin{cases}C \epsilon^{\frac{2}{k+2}} & \text { if } 0 \notin f(\mathbb{R}), \\ C \epsilon^{\min \left\{\frac{2}{k+2}, \frac{2}{3}\right\}} & \text { if } 0 \in f(\mathbb{R}) \backslash \operatorname{cv}(f) \\ C \epsilon^{\min \left\{\frac{2}{k+2}, \frac{1}{2}\right\}} & \text { if } 0 \in \operatorname{cv}(f)\end{cases}
$$

v) There exists $C>1$ such that $\kappa(\epsilon, \lambda) \leq C \epsilon^{\frac{2}{k+4}}$ for all $(\epsilon, \lambda) \in(0,1) \times \mathbb{R}$. Moreover, if $\kappa(\epsilon, \lambda) \geq C^{-1} \epsilon^{\frac{2}{k+4}}$, then $\lambda$ is comparable to $\epsilon^{-\frac{4}{k+4}}$.

Finally all estimates in $\boldsymbol{i}), \boldsymbol{i i}), \boldsymbol{i i i}), \boldsymbol{i v})$, and $\boldsymbol{v})$ are optimal, in the sense that one can find $\lambda=\lambda(\epsilon)$ so that the pair $(\epsilon, \lambda(\epsilon))$ satisfies the required conditions as $\epsilon \rightarrow 0$ and so that $\kappa(\epsilon, \lambda(\epsilon))$ is comparable to the upper bound in this limit.

Theorem 1.8 is of course a direct consequence of Proposition 4.1, since

$$
\Psi(\epsilon)=\min _{\lambda \in \mathbb{R}} \kappa(\epsilon, \lambda)^{-1} \in\left[M_{4}^{-1} \epsilon^{-\frac{2}{k+4}}, M_{4} \epsilon^{-\frac{2}{k+4}}\right], \quad \text { for some } M_{4} \geq 1 .
$$

Proposition 4.1 also allows to localize the pseudospectrum of $H_{\epsilon}$ accurately:

Corollary 4.2 The complex domain $\omega_{\epsilon}$ defined for $\epsilon \in(0,1]$ by

$$
\omega_{\epsilon}=\bigcup_{\lambda \in \mathbb{R}}\left\{z \in \mathbb{C} ;|z-i \lambda| \leq \frac{1}{2 \kappa(\epsilon, \lambda)}\right\} \cup\{z \in \mathbb{C} ; \operatorname{Re}(z) \leq 0\}
$$

avoids the pseudospectrum of $H_{\epsilon}$ as $\epsilon \rightarrow 0$, whereas the domain

$$
\tilde{\omega}_{\epsilon}=\left\{z \in \mathbb{C} ; \operatorname{Re}(z) \leq \Psi(\epsilon) \log \left(\epsilon^{-1}\right)^{2}\right\} \cap\left(\mathbb{C} \backslash \omega_{\epsilon}\right)
$$

meets the pseudospectrum of $H_{\epsilon}$ as $\epsilon \rightarrow 0$.

Proof: If $|z-i \lambda| \leq(2 \kappa(\epsilon, \lambda))^{-1}$, the resolvent formula shows that $\left\|\left(H_{\epsilon}-z\right)^{-1}\right\| \leq 2 \kappa(\epsilon, \lambda) \leq C$. Moreover, we know that $\left\|\left(H_{\epsilon}-z\right)^{-1}\right\| \leq 1$ if $\operatorname{Re}(z) \leq 0$, thus $\omega_{\epsilon}$ avoids the pseudospectrum of $H_{\epsilon}$ as $\epsilon \rightarrow 0$. Remark that $\omega_{\epsilon}$ contains the half-plane $\{z \in \mathbb{C} ; \operatorname{Re}(z) \leq \Psi(\epsilon) / 2\}$. On the other hand, if $\mu_{\epsilon}=\Psi(\epsilon) \log \left(\epsilon^{-1}\right)^{2}$, it follows from Proposition 4.1 that $\mu_{\epsilon} \gg \Psi(\epsilon)\left(1+\log \Psi(\epsilon)+\log \left(\epsilon^{-1}\right)\right)$ if $\epsilon$ is small, hence the half-plane $\left\{\operatorname{Re}(z) \leq \mu_{\epsilon}\right\}$ meets the pseudospectrum of $H_{\epsilon}$ by Lemma 1.3. The same conclusion holds of course for $\tilde{\omega}_{\epsilon}$. 


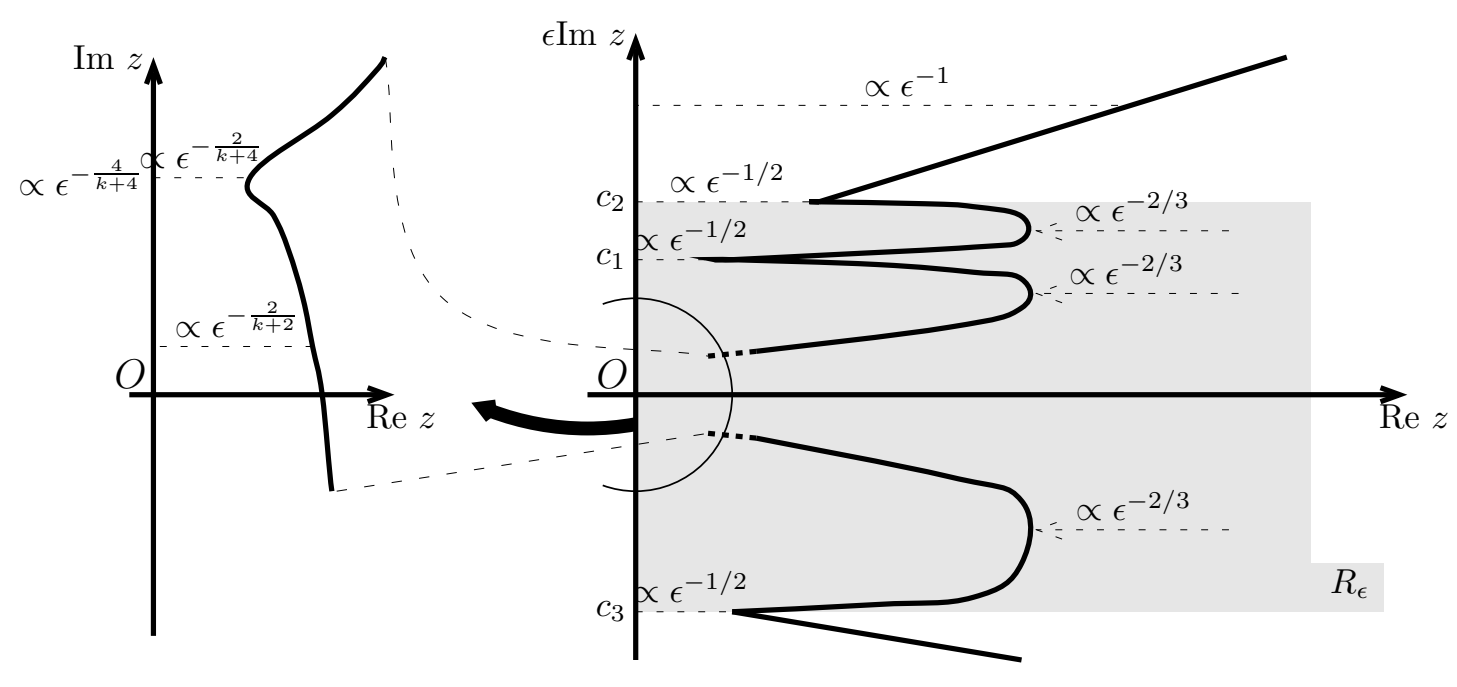

Fig. 2: The domain $\omega_{\epsilon}$ on the left-hand side of the solid curve avoids the pseudospectrum of $H_{\epsilon}$ as $\epsilon \rightarrow 0$. The picture on the right shows the geometry at the scale $\epsilon z=\mathcal{O}(1)$, while the left picture focuses on the region where $\epsilon z$ is small. Here $R_{\epsilon}=\{z \in \mathbb{C} ; \operatorname{Re} z \geq 0, \min f \leq \epsilon \operatorname{Im} z \leq \max f\}$ and $c v(f)=\left\{c_{1}, c_{2}, c_{3}\right\}$.

To prove Proposition 4.1, we start with a variant of the so-called IMS-localization formula, see [2].

Lemma 4.3 Consider a Schrödinger-type operator $Q=-\Delta+V$ in $\mathbb{R}^{d}$, where $V$ is a measurable function. Take a locally finite partition of unity $\left\{\chi_{j}\right\}_{j \in J}$, where $\chi_{j} \in C_{0}^{\infty}\left(\mathbb{R}^{d}, \mathbb{R}\right)$, such that

$$
\sum_{j \in J} \chi_{j}(x)^{2}=1, \quad \text { for all } x \in \mathbb{R}^{d}
$$

and

$$
m_{1}^{2} \stackrel{\text { def }}{=} \sup _{x \in \mathbb{R}^{d}} \sum_{j \in J}\left|\nabla \chi_{j}(x)\right|^{2}<+\infty, \quad m_{2}^{2} \stackrel{\text { def }}{=} \sup _{x \in \mathbb{R}^{d}} \sum_{j \in J}\left(\Delta \chi_{j}(x)\right)^{2}<+\infty .
$$

Then the estimate

$$
2\|Q u\|^{2}+3 m_{2}^{2}\|u\|^{2}+8 m_{1}^{2}\|\nabla u\|^{2} \geq \sum_{j \in J}\left\|Q \chi_{j} u\right\|^{2},
$$

holds for any $u \in C_{0}^{\infty}\left(\mathbb{R}^{d}\right)$. Moreover, if $\operatorname{Re} V \geq 0$, then

$$
2\|Q u\|^{2}+3 m_{2}^{2}\|u\|^{2}+8 m_{1}^{2} \operatorname{Re}\langle Q u, u\rangle \geq \sum_{j \in J}\left\|Q \chi_{j} u\right\|^{2} .
$$

Proof: For any $\chi \in C_{0}^{\infty}\left(\mathbb{R}^{d}, \mathbb{R}\right)$ we have

$$
\begin{aligned}
Q^{*} \chi^{2} Q & =Q^{*} \chi Q \chi+Q^{*} \chi[\chi, Q] \\
& =\chi Q^{*} Q \chi+\left[Q^{*}, \chi\right] Q \chi+Q^{*} \chi[\chi, Q] \\
& =\chi Q^{*} Q \chi+\left[Q^{*}, \chi\right] \chi Q+Q^{*} \chi[\chi, Q]+\left[Q^{*}, \chi\right][Q, \chi] \\
& =\chi Q^{*} Q \chi-[\Delta, \chi] \chi Q+Q^{*} \chi[\Delta, \chi]+[\Delta, \chi][\Delta, \chi]
\end{aligned}
$$


Since

$$
[\Delta, \chi]=2(\nabla \chi) \cdot \nabla+(\Delta \chi)=2 \nabla \cdot(\nabla \chi)-(\Delta \chi)
$$

this implies

$$
Q^{*} \chi^{2} Q=\chi Q^{*} Q \chi-\nabla \cdot\left(\nabla \chi^{2}\right) Q+(\Delta \chi) \chi Q+Q^{*}\left(\nabla \chi^{2}\right) \cdot \nabla+Q^{*} \chi(\Delta \chi)-R_{\chi}^{*} R_{\chi},
$$

with $R_{\chi}=2(\nabla \chi) \cdot \nabla+(\Delta \chi)$. We now apply this identity with $\chi=\chi_{j}$ and sum over $j \in J$. In view of (4.2), the left-hand side reduces to $Q^{*} Q$, and the second and fourth terms in the right-hand side disappear, so that

$$
Q^{*} Q=\sum_{j \in J} \chi_{j} Q^{*} Q \chi_{j}+\sum_{j \in J}\left(\left(\Delta \chi_{j}\right) \chi_{j} Q+Q^{*} \chi_{j}\left(\Delta \chi_{j}\right)\right)-\sum_{j \in J} R_{\chi_{j}}^{*} R_{\chi_{j}} .
$$

Thus, for any $u \in C_{0}^{\infty}\left(\mathbb{R}^{d}, \mathbb{R}\right)$, we have

$$
\begin{aligned}
\|Q u\|^{2} & \geq \sum_{j \in J}\left(\left\|Q \chi_{j} u\right\|^{2}-\left(\left\|\chi_{j} Q u\right\|^{2}+\left\|\left(\Delta \chi_{j}\right) u\right\|^{2}\right)-\left(8\left\|\left(\nabla \chi_{j}\right) \cdot \nabla u\right\|^{2}+2\left\|\left(\Delta \chi_{j}\right) u\right\|^{2}\right)\right) \\
& \geq\left(\sum_{j \in J}\left\|Q \chi_{j} u\right\|^{2}\right)-\|Q u\|^{2}-3 m_{2}^{2}\|u\|^{2}-8 m_{1}^{2}\|\nabla u\|^{2}
\end{aligned}
$$

which is (4.4). When $\operatorname{Re} V \geq 0$, the inequality $\|\nabla u\|^{2} \leq \operatorname{Re}\langle Q u, u\rangle$ implies (4.5).

The idea is now to apply Lemma 4.3 to the one-dimensional operator $H_{\epsilon}-i \lambda$, using a dyadic partition of unity. This allows us to reduce a global problem on the whole real line to a family of compactly supported problems, indexed by the parameter $j \in \mathbb{N}$. The choice of a dyadic partition is convenient to take into account the precise behavior of the coefficients of $H_{\epsilon}$ as $x \rightarrow \pm \infty$.

Lemma 4.4 For $j \in \mathbb{N}, \epsilon>0$, and $\lambda \in \mathbb{R}$, consider the operator

$$
P_{j, \epsilon, \lambda}=-2^{-2 j} \partial_{x}^{2}+2^{2 j} x^{2}+\frac{i}{\epsilon} f\left(2^{j} x\right)-i \lambda
$$

and let

$$
C_{j}(\epsilon, \lambda)=\inf \left\{\left\|P_{j, \epsilon, \lambda} u\right\| ; u \in C_{0}^{\infty}(\mathbb{R}), \operatorname{supp} u \subset K_{j},\|u\|=1\right\},
$$

where $K_{0}=[-1,1]$ and $K_{j}=[-1,-1 / 4] \cup[1 / 4,1]$ for any $j>0$. Then the quantity $\kappa(\epsilon, \lambda)=$ $\left\|\left(H_{\epsilon}-i \lambda\right)^{-1}\right\|$ satisfies

$$
\left(\inf _{j \in \mathbb{N}} C_{j}(\epsilon, \lambda)\right)^{-1} \leq \kappa(\epsilon, \lambda) \leq C\left(\inf _{j \in \mathbb{N}} C_{j}(\epsilon, \lambda)\right)^{-1}
$$

for some constant $C \geq 1$ independent of $\epsilon, \lambda$.

Remark 4.5 It is clear that $C_{j}(\epsilon, \lambda) \geq 1$ for all $j \in \mathbb{N}, \epsilon>0, \lambda \in \mathbb{R}$, because

$$
\|u\|^{2} \leq \operatorname{Re}\left\langle P_{j, \epsilon, \lambda} u, u\right\rangle \leq\left\|P_{j, \epsilon, \lambda} u\right\|\|u\|,
$$

for all $u \in C_{0}^{\infty}(\mathbb{R})$. 
Proof: We choose a dyadic partition of unity $\left\{\chi_{j}\right\}_{j \in \mathbb{N}}$ such that

$$
1=\sum_{j=0}^{\infty} \chi_{j}(x)^{2}=\chi_{0}(x)^{2}+\sum_{j=1}^{\infty} \tilde{\chi}\left(\frac{x}{2^{j}}\right)^{2}
$$

where $\chi_{0}, \tilde{\chi} \in C_{0}^{\infty}(\mathbb{R})$ satisfy

$$
\chi_{0}(x)=\left\{\begin{array}{ll}
1 & \text { if }|x| \leq \frac{3}{4}, \\
0 & \text { if }|x| \geq 1,
\end{array} \quad \tilde{\chi}(x)=\left\{\begin{array}{lll}
1 & \text { if } \quad \frac{1}{2} \leq|x| \leq \frac{3}{4} \\
0 & \text { if } \quad|x| \leq \frac{3}{8} \text { or }|x| \geq 1 .
\end{array}\right.\right.
$$

It is clear that such a partition exists and that the quantities $m_{1}^{2}, m_{2}^{2}$ defined by (4.3) are finite. Thus we can apply Lemma 4.3 to the operator $Q=H_{\epsilon}-i \lambda=-\partial_{x}^{2}+x^{2}+\frac{i}{\epsilon} f(x)-i \lambda$, for any $\epsilon>0$ and $\lambda \in \mathbb{R}$. Since $\|u\|^{2} \leq \operatorname{Re}\langle Q u, u\rangle \leq\|Q u\|\|u\| \leq\|Q u\|^{2}$ for all $u \in C_{0}^{\infty}(\mathbb{R})$, it follows from (4.5) that

$$
C^{2}\|Q u\|^{2} \geq \sum_{j=0}^{\infty}\left\|Q \chi_{j} u\right\|^{2}
$$

where $C^{2}=2+8 m_{1}^{2}+3 m_{2}^{2}$. Now, for any $j \in \mathbb{N}$, we define

$$
v_{j}(x)=2^{j / 2} \chi_{j}\left(2^{j} x\right) u\left(2^{j} x\right), \quad x \in \mathbb{R},
$$

so that $\operatorname{supp} v_{j} \subset \operatorname{supp} \chi_{j}\left(2^{j} \cdot\right) \subset K_{j}$ and $\left(P_{j, \epsilon, \lambda} v_{j}\right)(x)=2^{j / 2}\left(Q \chi_{j} u\right)\left(2^{j} x\right)$. If we denote $m(\epsilon, \lambda)=$ $\inf _{j \in \mathbb{N}} C_{j}(\epsilon, \lambda)$, we thus find

$$
C^{2}\|Q u\|^{2} \geq \sum_{j=0}^{\infty}\left\|P_{j, \epsilon, \lambda} v_{j}\right\|^{2} \geq m(\epsilon, \lambda)^{2} \sum_{j=0}^{\infty}\left\|v_{j}\right\|^{2} \geq m(\epsilon, \lambda)^{2}\|u\|^{2},
$$

because $\sum_{j}\left\|v_{j}\right\|^{2}=\sum_{j}\left\|\chi_{j} u\right\|^{2}=\|u\|^{2}$. Since $Q=H_{\epsilon}-i \lambda$ and $C_{0}^{\infty}(\mathbb{R})$ is dense in $L^{2}(\mathbb{R})$, it follows from (4.9) that $\kappa(\epsilon, \lambda)=\left\|\left(H_{\epsilon}-i \lambda\right)^{-1}\right\| \leq C / m(\epsilon, \lambda)$, which is the upper bound in (4.8).

To prove the lower bound, we fix $\delta>0, \epsilon>0, \lambda \in \mathbb{R}$, and we take $j \in \mathbb{N}, v_{j} \in C_{0}^{\infty}(\mathbb{R})$ such that $v_{j} \not \equiv 0, \operatorname{supp} v_{j} \subset K_{j}$, and $\left\|P_{j, \epsilon, \lambda} v_{j}\right\| \leq(m(\epsilon, \lambda)+\delta)\left\|v_{j}\right\|$. Setting $v_{j}(x)=2^{j / 2} u\left(2^{j} x\right)$, we find that $\|Q u\| \leq(m(\epsilon, \lambda)+\delta)\|u\|$, hence $\kappa(\epsilon, \lambda) \geq(m(\epsilon, \lambda)+\delta)^{-1}$. Since $\delta>0$ was arbitrary, this concludes the proof.

\section{Proof of Proposition 4.1;}

The proof of assertion $\mathbf{i}$ ) is easy: if $\operatorname{dist}(\epsilon \lambda, f(\mathbb{R})) \geq \delta$, then $\operatorname{dist}\left(i \lambda, \Theta\left(H_{\epsilon}\right)\right) \geq \delta / \epsilon$, where $\Theta\left(H_{\epsilon}\right)$ is the numerical range defined in (1.5). The last inequality in (1.9) then implies $\kappa(\epsilon, \lambda) \leq \epsilon / \delta$.

In the remaining four cases, we start from Lemma 4.4 and use (4.8) to bound $\kappa(\epsilon, \lambda)$. It turns out to be convenient to rewrite the operator $P_{j, \epsilon, \lambda}$ in the equivalent form

$$
P_{j, \epsilon, \lambda}=\frac{1}{\epsilon 2^{k j}}\left[-\epsilon 2^{(k-2) j} \partial_{x}^{2}+\epsilon 2^{(k+2) j} x^{2}+i\left(2^{k j} f\left(2^{j} x\right)-2^{k j} \epsilon \lambda\right)\right],
$$

where $k>0$ is the parameter that governs the asymptotic behavior of $f(x)$ as $|x| \rightarrow \infty$, see Hypothesis 1.6, For later use, we observe that

$$
C_{f} \stackrel{\text { def }}{=} \sup _{j \in \mathbb{N}} \sup _{x \in K_{j}} 2^{k j}\left|f\left(2^{j} x\right)\right|<+\infty
$$


ii) Suppose that $\operatorname{dist}(\epsilon \lambda, \operatorname{cv}(f) \cup\{0\}) \geq \delta$. Without loss of generality, we also assume that $\epsilon|\lambda| \leq\|f\|_{L^{\infty}}+\delta$, because otherwise we can use the estimate established in i). For any $u \in C_{0}^{\infty}(\mathbb{R})$ with $\operatorname{supp} u \in K_{j}$ and $u \not \equiv 0$, we have the lower bound

$$
\frac{\left\|P_{j, \epsilon, \lambda} u\right\|}{\|u\|} \geq \frac{\left|\operatorname{Im}\left\langle P_{j, \epsilon, \lambda} u, u\right\rangle\right|}{\|u\|^{2}}=\frac{\left|\left\langle\left[2^{k j} f\left(2^{j} \cdot\right)-2^{k j} \epsilon \lambda\right] u, u\right\rangle\right|}{\epsilon 2^{k j}\|u\|^{2}} \geq \frac{1}{\epsilon}\left(\epsilon|\lambda|-\frac{C_{f}}{2^{k j}}\right),
$$

where $C_{f}$ is given by (4.11). Since $\epsilon|\lambda| \geq \delta$, we deduce that $C_{j}(\epsilon, \lambda) \geq \delta /(2 \epsilon)$ whenever $j$ is large enough so that $2^{k j} \geq 2 C_{f} / \delta$. Thus only a finite number of indices $j$ have to be considered, and the problem is therefore reduced to finding a lower bound on the quantity $\left\|\left(H_{\epsilon}-i \lambda\right) u\right\|$ when $u \in C_{0}^{\infty}\left(\left\{x \in \mathbb{R} ;|x|<R_{\delta}\right\}\right)$, for some $R_{\delta}>0$. On a bounded domain, we can drop the bounded term $x^{2}$ in $H_{\epsilon}$ and only consider the operator $Q=-\partial_{x}^{2}+\frac{i}{\epsilon}(f(x)-\epsilon \lambda)$. Take two functions $\theta_{0}, \theta_{1} \in C^{\infty}(\mathbb{R}, \mathbb{R})$ such that $\operatorname{supp} \theta_{0} \subset[-2,2], \operatorname{supp} \theta_{1} \subset(-\infty,-1] \cup[1,+\infty)$, and $\theta_{0}(x)^{2}+\theta_{1}(x)^{2}=1$ for all $x \in \mathbb{R}$. Given $\sigma>0$ which will be fixed below, we consider the new partition of unity

$$
\chi_{0}(x)^{2}+\chi_{1}(x)^{2} \equiv 1, \quad \text { with } \quad \chi_{j}(x)=\theta_{j}\left(\frac{f(x)-\epsilon \lambda}{\epsilon^{\sigma}}\right) \quad \text { for } \quad j=0,1 .
$$

The quantities (4.3) for this partition satisfy $m_{1}^{2} \leq C_{1} \epsilon^{-2 \sigma}$ and $m_{2}^{2} \leq C_{1} \epsilon^{-4 \sigma}$ for some $C_{1}>0$, hence (4.5) implies

$$
2\|Q u\|^{2}+\frac{3 C_{1}}{\epsilon^{4 \sigma}}\|u\|^{2}+\frac{8 C_{1}}{\epsilon^{2 \sigma}}\|Q u\|\|u\| \geq\left\|Q\left(\chi_{0} u\right)\right\|^{2}+\left\|Q\left(\chi_{1} u\right)\right\|^{2} .
$$

The last term is easily bounded from below using the support condition on $\chi_{1}$, which yields

$$
\left\|\chi_{1} u\right\|\left\|Q\left(\chi_{1} u\right)\right\| \geq \frac{1}{\epsilon}\left|\left\langle(f(x)-\lambda \epsilon) \chi_{1} u, \chi_{1} u\right\rangle\right| \geq \epsilon^{\sigma-1}\left\|\chi_{1} u\right\|^{2} .
$$

To bound $Q\left(\chi_{0} u\right)$, we observe that $f^{-1}(\epsilon \lambda)$ is a finite set $\left\{x_{1}, \ldots, x_{n}\right\}$ because $f$ has a only a finite number of critical points. Since $\operatorname{dist}(\epsilon \lambda, \operatorname{cv}(f) \cup\{0\}) \geq \delta$, it follows that for $\epsilon>0$ small enough the support of $\chi_{0}$ is the disjoint union of $n$ intervals $I_{1}, \ldots, I_{n}$ of size $\mathcal{O}\left(\epsilon^{\sigma}\right)$ centered at the points $x_{1}, \ldots, x_{n}$. In particular, $\chi_{0} u=\sum_{\ell=1}^{n} u_{\ell}$ with $\operatorname{supp} u_{\ell} \cap \operatorname{supp} u_{\ell^{\prime}}=\emptyset$ when $\ell \neq \ell^{\prime}$. Inside $I_{\ell}$, the operator $Q$ is well approximated by $Q_{\ell}=-\partial_{x}^{2}+\frac{i}{\epsilon} f^{\prime}\left(x_{\ell}\right)\left(x-x_{\ell}\right)$, because

$$
\begin{aligned}
\left\|Q u_{\ell}\right\|^{2} & \geq \frac{1}{2}\left\|Q_{\ell} u_{\ell}\right\|^{2}-\left\|\frac{f(x)-f\left(x_{\ell}\right)-f^{\prime}\left(x_{\ell}\right)\left(x-x_{\ell}\right)}{\epsilon} u_{\ell}\right\|^{2} \\
& \geq \frac{1}{2}\left\|Q_{\ell} u_{\ell}\right\|^{2}-\frac{C_{2}\left\|f^{\prime \prime}\right\|_{L^{\infty}}^{2} \epsilon^{4 \sigma}}{\epsilon^{2}}\left\|u_{\ell}\right\|^{2} .
\end{aligned}
$$

On the other hand, the operator $Q_{\ell}$ is unitarily equivalent to the microlocal model

$$
\tilde{Q}_{\gamma}=\gamma^{2 / 3}\left(-\partial_{y}^{2} \pm i y\right), \quad \text { where } \quad \gamma=\frac{\left|f^{\prime}\left(x_{\ell}\right)\right|}{\epsilon},
$$

which satisfies $\left\|\tilde{Q}_{\gamma} u\right\| \geq C \gamma^{2 / 3}\|u\|$. Actually, the operator $P=-\partial_{y}^{2} \pm i y$ has a compact resolvent, since

$$
\|P u\|^{2}=\left\|\partial_{y}^{2} u\right\|^{2}+\|y u\|^{2} \pm 2 i\left\langle u, \partial_{y} u\right\rangle \geq \frac{1}{2}\left\|\partial_{y}^{2} u\right\|^{2}+\|y u\|^{2}-C\|u\|^{2},
$$

with an empty kernel $\left(P u=0\right.$ implies $\left.\left\|\partial_{y} u\right\|^{2}=\operatorname{Re}\langle P u, u\rangle=0\right)$, and it is therefore invertible. We refer to Chap. 27 in [16] or to [4] for a geometric analysis of similar and more general models. Alternatively, one can use the Lie-algebra approach developed in [20], 11] if one sets 
$-\partial_{y}^{2}+y \partial_{t}=-X_{1}^{2}+X_{0}$ with $X_{1}=\partial_{y}, X_{0}=y \partial_{t}$ and if one considers the value $\tau=1$ for the frequency variable $\tau$ dual to $t \in \mathbb{R}$. Summarizing, we have shown that

$$
\left\|Q\left(\chi_{0} u\right)\right\|^{2}=\sum_{\ell=1}^{n}\left\|Q u_{\ell}\right\|^{2} \geq\left(C_{3} \epsilon^{-4 / 3}-C_{2}\left\|f^{\prime \prime}\right\|_{L^{\infty}}^{2} \epsilon^{4 \sigma-2}\right)\left\|\chi_{0} u\right\|^{2},
$$

for some $C_{3}>0$. We now assume that $\sigma>1 / 6$, so that $\epsilon^{4 \sigma-2} \ll \epsilon^{-4 / 3}$ if $\epsilon$ is small. Replacing (4.14), (4.17) into (4.13), we thus find

$$
C_{4}\|Q u\|^{2}+\frac{C_{4}}{\epsilon^{4 \sigma}}\|u\|^{2} \geq C_{4}^{-1} \min \left\{\epsilon^{2 \sigma-2}, \epsilon^{-4 / 3}\right\}\|u\|^{2},
$$

for some $C_{4}>0$. Finally, we suppose that $\sigma<1 / 3$, so that $\epsilon^{-4 \sigma} \ll \epsilon^{-4 / 3} \ll \epsilon^{2 \sigma-2}$. We thus obtain the estimate $2 C_{4}^{2}\|Q u\|^{2} \geq \epsilon^{-4 / 3}\|u\|^{2}$, which proves that $\kappa(\epsilon, \lambda) \leq C \epsilon^{2 / 3}$.

iii) The assumption $\lim _{\epsilon \rightarrow 0} \epsilon \lambda=c \in \operatorname{cv}(f) \backslash\{0\}$ implies that $\epsilon|\lambda| \geq \delta$ for some fixed $\delta>0$ if $\epsilon$ is small enough. Thus we can reduce the analysis to a bounded spatial domain like in case ii). By assumption $f^{-1}(c)$ is a finite set which contains at least one critical point of $f$, but in general this set contains noncritical points as well. Using a partition of unity, we can treat the noncritical points separately and estimate their contributions as in case ii). So, for simplicity, we assume here that $f^{-1}(c)$ consists of critical points only. We shall consider two different regimes, depending on how fast $\epsilon \lambda$ converges to $c$ as $\epsilon \rightarrow 0$, and then check after some iterations that they provide a complete information for the general assumption $\lim _{\epsilon \rightarrow 0} \epsilon \lambda=c$.

a) We first study the case when $\epsilon \lambda$ converges slowly to $c$. More precisely, we assume that

$$
\epsilon^{\sigma_{1}} \leq|\epsilon \lambda-c| \leq \epsilon^{\sigma_{2}}
$$

where $0 \leq \sigma_{2}<\sigma_{1}<1 / 2$ and $3 \sigma_{2}>5 \sigma_{1}-1$. If $\sigma_{2}=0$, we also suppose that $\epsilon \lambda \rightarrow c$ as $\epsilon \rightarrow 0$. Several intervals $\left[\sigma_{2}, \sigma_{1}\right]$ will be fixed iteratively in step c) below. Our goal is to show that $\|Q u\| \geq C \epsilon^{-1 / 2}\|u\|$ for all $u \in C_{0}^{\infty}(\mathbb{R})$, where $Q=-\partial_{x}^{2}+\frac{i}{\epsilon}(f(x)-\epsilon \lambda)$. We choose $\sigma \in\left(\sigma_{1}, 1 / 2\right)$ such that

$$
\frac{2 \sigma_{1}}{3}+\frac{1}{6}<\sigma<\frac{1}{3}+\frac{\sigma_{1}}{3}-\frac{\sigma_{1}-\sigma_{2}}{2},
$$

and we use again the partition of unity defined by (4.12). If $x \in \operatorname{supp} \chi_{0}$ or $x \in \operatorname{supp} \chi_{1}^{\prime}$, we have $|f(x)-\epsilon \lambda| \leq 2 \epsilon^{\sigma}$ and using (4.18) we easily find

$$
\frac{\epsilon^{\sigma_{1} / 2}}{C_{2}} \leq \frac{|f(x)-c|^{1 / 2}}{C_{1}} \leq\left|f^{\prime}(x)\right| \leq C_{1}|f(x)-c|^{1 / 2} \leq C_{2} \epsilon^{\sigma_{2} / 2}
$$

for some $C_{1}, C_{2}>0$. These estimates allow to give precise bounds on the quantities $m_{1}^{2}$ and $m_{2}^{2}$ defined in (4.3). Since

$$
\begin{aligned}
& \chi_{j}^{\prime}(x)=\frac{f^{\prime}(x)}{\epsilon^{\sigma}} \theta_{j}^{\prime}\left(\frac{f(x)-\lambda \epsilon}{\epsilon^{\sigma}}\right), \\
& \chi_{j}^{\prime \prime}(x)=\frac{f^{\prime \prime}(x)}{\epsilon^{\sigma}} \theta_{j}^{\prime}\left(\frac{f(x)-\lambda \epsilon}{\epsilon^{\sigma}}\right)+\left(\frac{f^{\prime}(x)}{\epsilon^{\sigma}}\right)^{2} \theta_{j}^{\prime \prime}\left(\frac{f(x)-\epsilon \lambda}{\epsilon^{\sigma}}\right), \quad j=0,1,
\end{aligned}
$$

we obtain

$$
m_{1}^{2} \leq C_{3} \epsilon^{\sigma_{2}-2 \sigma}, \quad \text { and } \quad m_{2}^{2} \leq C_{3}^{2}\left(\epsilon^{-2 \sigma}+\epsilon^{2 \sigma_{2}-4 \sigma}\right) \leq 2 C_{3}^{2} \epsilon^{2 \sigma_{2}-4 \sigma},
$$

for some $C_{3}>0$, and it follows from (4.5) that

$$
3\|Q u\|^{2}+22 C_{3}^{2} \epsilon^{2 \sigma_{2}-4 \sigma}\|u\|^{2} \geq\left\|Q\left(\chi_{0} u\right)\right\|^{2}+\left\|Q\left(\chi_{1} u\right)\right\|^{2} .
$$


By (4.14) we have $\left\|Q\left(\chi_{1} u\right)\right\| \geq \epsilon^{\sigma-1}\left\|\chi_{1} u\right\|$, and to bound $\left\|Q\left(\chi_{0} u\right)\right\|$ we proceed as in case ii). Denoting $f^{-1}(\epsilon \lambda)=\left\{x_{1}, \ldots, x_{n}\right\}$, we decompose as before $\chi_{0} u=\sum_{\ell=1}^{n} u_{\ell}$, and we observe that every $x \in \operatorname{supp} u_{\ell}$ satisfies $\left|x-x_{\ell}\right| \leq 2 C_{2} \epsilon^{\sigma-\sigma_{1} / 2}$, because

$$
2 \epsilon^{\sigma} \geq|f(x)-\epsilon \lambda| \geq\left|x-x_{\ell}\right| \inf \left\{\left|f^{\prime}(y)\right| ; y \in \operatorname{supp} u_{\ell}\right\} \geq C_{2}^{-1} \epsilon^{\sigma_{1} / 2}\left|x-x_{\ell}\right| .
$$

As in (4.15), we thus find

$$
\left\|Q u_{\ell}\right\|^{2} \geq\left(\frac{C\left|f^{\prime}\left(x_{\ell}\right)\right|^{4 / 3}}{\epsilon^{4 / 3}}-\frac{4 C_{2}^{4}\left\|f^{\prime \prime}\right\|_{L^{\infty}}^{2} \epsilon^{4 \sigma-2 \sigma_{1}}}{\epsilon^{2}}\right)\left\|u_{\ell}\right\|^{2} \geq C_{4} \epsilon^{\frac{2 \sigma_{1}}{3}-\frac{4}{3}}\left\|u_{\ell}\right\|^{2}
$$

for some $C_{4}>0$, because $\epsilon^{-4 / 3}\left|f^{\prime}\left(x_{\ell}\right)\right|^{4 / 3} \geq C_{2}^{-4 / 3} \epsilon^{\frac{2 \sigma_{1}}{3}-\frac{4}{3}} \gg \epsilon^{4 \sigma-2 \sigma_{1}-2}$ by (4.19). Summing over $\ell$ as in (4.17), we obtain the desired lower bound on $\left\|Q\left(\chi_{0} u\right)\right\|^{2}$, and returning to (4.20) we arrive at

$$
C_{5}\|Q u\|^{2}+C_{5} \epsilon^{2 \sigma_{2}-4 \sigma}\|u\|^{2} \geq C_{5}^{-1} \min \left\{\epsilon^{2 \sigma-2}, \epsilon^{\frac{2 \sigma_{1}}{3}-\frac{4}{3}}\right\}\|u\|^{2},
$$

for some $C_{5}>0$. Since $\epsilon^{2 \sigma_{2}-4 \sigma} \ll \epsilon^{\frac{2 \sigma_{1}}{3}-\frac{4}{3}} \ll \epsilon^{2 \sigma-2}$ by (4.19), we conclude that

$$
2 C_{5}^{2}\|Q u\|^{2} \geq \epsilon^{\frac{2 \sigma_{1}}{3}-\frac{4}{3}}\|u\|^{2} \geq \epsilon^{-1}\|u\|^{2}, \quad \text { for all } u \in C_{0}^{\infty}(\mathbb{R}) .
$$

b) We now assume that $|\epsilon \lambda-c| \leq \epsilon^{\sigma}$, for some $\sigma \in\left(\frac{1}{3}, \frac{1}{2}\right)$, and we use again the partition of unity defined by (4.12). As before, we have $\left|f^{\prime}(x)\right| \leq C_{1}|f(x)-c|^{1 / 2} \leq C_{2} \epsilon^{\sigma / 2}$ for all $x \in \operatorname{supp} \chi_{0}$ and all $x \in \operatorname{supp} \chi_{1}^{\prime}$, and it follows that the quantities (4.3) satisfy $m_{1}^{2} \leq C_{3} \epsilon^{-\sigma}, m_{2}^{2} \leq C_{3}^{2} \epsilon^{-2 \sigma}$. Thus (4.5) becomes

$$
3\|Q u\|^{2}+22 C_{3}^{2} \epsilon^{-2 \sigma}\|u\|^{2} \geq\left\|Q\left(\chi_{0} u\right)\right\|^{2}+\left\|Q\left(\chi_{1} u\right)\right\|^{2} \geq\left\|Q\left(\chi_{0} u\right)\right\|^{2}+\epsilon^{2 \sigma-2}\left\|\chi_{1} u\right\|^{2} .
$$

Since $\epsilon^{-2 \sigma} \ll \epsilon^{-1} \ll \epsilon^{2 \sigma-2}$, it is sufficient to show that $\left\|Q\left(\chi_{0} u\right)\right\|^{2} \geq C \epsilon^{-1}\left\|\chi_{0} u\right\|^{2}$. To this end, we consider the set $f^{-1}(c)=\left\{x_{1}, \ldots, x_{n}\right\}$ and we decompose as before $\chi_{0} u=\sum_{\ell=1}^{n} u_{\ell}$. In the support of $u_{\ell}$, we can use the quadratic approximation

$$
Q_{\ell}=-\partial_{x}^{2}+\frac{i}{\epsilon}\left[\frac{f^{\prime \prime}\left(x_{\ell}\right)}{2}\left(x-x_{\ell}\right)^{2}-(\epsilon \lambda-c)\right] .
$$

Indeed, if $x \in \operatorname{supp} u_{\ell}$, then $\left|x-x_{\ell}\right| \leq C_{4} \epsilon^{\sigma / 2}$ for some $C_{4}>0$, and it follows that

$$
\left\|Q u_{\ell}\right\|^{2} \geq \frac{1}{2}\left\|Q_{\ell} u_{\ell}\right\|^{2}-\frac{C_{4}^{6}\left\|f^{\prime \prime \prime}\right\|_{L^{\infty}}^{2} \epsilon^{3 \sigma}}{36 \epsilon^{2}}\left\|u_{\ell}\right\|^{2}=\frac{1}{2}\left\|Q_{\ell} u_{\ell}\right\|^{2}-C_{5} \epsilon^{3 \sigma-2}\left\|u_{\ell}\right\|^{2}
$$

for some $C_{5}>0$. On the other hand, the operator $Q_{\ell}$ is unitarily equivalent to the microlocal model

$$
\gamma^{1 / 2}\left(-\partial_{y}^{2} \pm i y^{2}-i \mu\right), \quad \text { where } \quad \gamma=\frac{\left|f^{\prime \prime}\left(x_{\ell}\right)\right|}{2 \epsilon} \quad \text { and } \quad \mu \gamma^{1 / 2}=\frac{\epsilon \lambda-c}{\epsilon} .
$$

Using the methods presented in [16] (Chap. 27), [1], 44, or even reproducing the analysis of ii) leading to (4.16), we find

$$
\left\|\left(-\partial_{y}^{2} \pm i y^{2}-i \mu\right) v\right\| \geq C\left(1+|\mu|^{1 / 3}\right)\|v\| \geq C\|v\|, \quad \text { for all } v \in C_{0}^{\infty}(\mathbb{R}) .
$$

Since $\epsilon^{3 \sigma-2} \ll \epsilon^{-1}$, this shows that $\left\|Q u_{\ell}\right\|^{2} \geq C \epsilon^{-1}\left\|u_{\ell}\right\|^{2}$, hence also $\left\|Q\left(\chi_{0} u\right)\right\|^{2} \geq C \epsilon^{-1}\left\|\chi_{0} u\right\|^{2}$. As in step a), we conclude that $\|Q u\|^{2} \geq C \epsilon^{-1}\|u\|^{2}$ for all $u \in C_{0}^{\infty}(\mathbb{R})$.

c) Take $\sigma_{1}^{0}=\frac{11}{30} \in\left(\frac{1}{3}, \frac{1}{2}\right)$ and, for any $n \in \mathbb{N}$, let $\sigma_{2}^{n}=\sigma_{1}^{n+1}=\frac{11}{6} \sigma_{1}^{n}-\frac{1}{3}$. Since $\frac{11}{6}>1$ and since the solution to $x=\frac{11}{6} x-\frac{1}{3}$ is $x=\frac{2}{5}>\sigma_{1}^{0}$, it is clear that $\sigma_{2}^{n}<\sigma_{1}^{n}$ for all $n$ and $\sigma_{2}^{n} \rightarrow-\infty$ 
as $n \rightarrow \infty$. Let $n_{0} \in \mathbb{N}$ be the smallest integer for which $\sigma_{2}^{n} \leq 0$. As $\frac{11}{6}>\frac{5}{3}$, the condition $3 \sigma_{2}^{n}>5 \sigma_{1}^{n}-1$ is satisfied for all $n \leq n_{0}$. Applying step a) to all intervals $\left[\max \left\{0, \sigma_{2}^{n}\right\}, \sigma_{1}^{n}\right]$ for $n=0, \ldots, n_{0}$, we obtain the lower bound $\left\|\left(H_{\epsilon}-i \lambda\right) u\right\| \geq C \epsilon^{-1 / 2}\|u\|$ whenever $\lambda$ satisfies (4.18) with $\left[\sigma_{2}, \sigma_{1}\right] \subset\left[0, \frac{11}{30}\right]$. In other words, there exists a constant $K>0$ such that

$$
\left(\begin{array}{l}
|\epsilon \lambda(\epsilon)-c| \geq \epsilon^{\frac{11}{30}} \\
\lim _{\epsilon \rightarrow 0} \epsilon \lambda(\epsilon)=c
\end{array}\right) \Rightarrow\left(\limsup _{\epsilon \rightarrow 0} \epsilon^{-1 / 2} \kappa(\epsilon, \lambda(\epsilon)) \leq K\right)
$$

Meanwhile step b) provides the same conclusion (possibly with another constant $K$ ) under the assumption $|\epsilon \lambda(\epsilon)-c| \leq \epsilon^{\frac{11}{30}}$. This concludes the proof of case iii).

iv) We consider the operator $P_{j, \epsilon, \lambda}$ given by (4.10) for $\lambda=0$ and $j \geq 1$. For any $u \in C_{0}^{\infty}(\mathbb{R})$ with $\operatorname{supp} u \subset K_{j}=\left\{\frac{1}{4} \leq|x| \leq 1\right\}$, we have

$$
\begin{aligned}
& \|u\|\left\|P_{j, \epsilon, 0} u\right\| \geq\left|\operatorname{Re}\left\langle P_{j, \epsilon, 0} u, u\right\rangle\right| \geq 2^{2 j} \int_{K_{j}} x^{2}|u(x)|^{2} \mathrm{x} \geq 2^{2 j-4}\|u\|^{2}, \\
& \|u\|\left\|P_{j, \epsilon, 0} u\right\| \geq\left|\operatorname{Im}\left\langle P_{j, \epsilon, 0} u, u\right\rangle\right| \geq \frac{1}{\epsilon 2^{k j}} \int_{K_{j}} 2^{k j}\left|f\left(2^{j} x\right)\left\|\left.u\right|^{2} \mathrm{x} \geq \frac{m_{j}}{\epsilon 2^{k j}}\right\| u \|^{2},\right.
\end{aligned}
$$

where $m_{j}=\inf \left\{2^{k j}\left|f\left(2^{j} x\right)\right| ; \frac{1}{4} \leq|x| \leq 1\right\}$. From Hypothesis 1.6 we know that $m_{j} \rightarrow 1$ as $j \rightarrow \infty$, so we can take $J \in \mathbb{N}$ large enough so that $m_{j} \geq 1 / 2$ whenever $j \geq J$. Thus

$$
C_{j}(\epsilon, \lambda) \geq \frac{1}{2}\left(2^{2 j-4}+\frac{1}{\epsilon 2^{k j+1}}\right) \geq C \epsilon^{-\frac{2}{k+2}}, \text { for all } j \geq J .
$$

The case when $j \in\{0, \ldots, J\}$ corresponds to a bounded spatial domain, and can be treated exactly as in ii) and iii). We find that $\left\|H_{\epsilon} u\right\| \geq C \epsilon^{-\sigma}\|u\|$ where $\sigma=1$ if $f^{-1}(0)=\emptyset, \sigma=2 / 3$ if $0 \in f(\mathbb{R}) \backslash \operatorname{cv}(f)$, and $\sigma=1 / 2$ if $0 \in \operatorname{cv}(f)$.

v) We want to show that $\kappa(\epsilon, \lambda) \leq C_{1} \epsilon^{\frac{2}{k+4}}$ for all $(\epsilon, \lambda) \in(0,1) \times \mathbb{R}$. In view of Lemma 4.4, we have to verify that

$$
C_{j}(\epsilon, \lambda) \geq C_{2} \epsilon^{-\frac{2}{k+4}}, \quad \text { for all } j \in \mathbb{N} \text { and all }(\epsilon, \lambda) \in(0,1) \times \mathbb{R} .
$$

As was already observed, the analysis of $C_{j}(\epsilon, \lambda)$ for $j$ in a finite set $\{0, \ldots, J\}$ can be performed as in ii), iii) and yields (in the worst case) the lower bound $C_{j}(\epsilon, \lambda) \geq C_{J} \epsilon^{-1 / 2}$, which is already better than (4.21). Thus is it sufficient to consider large values of $j$. Fix such a $j$ and choose $u \in C_{0}^{\infty}(\mathbb{R})$ such that $\operatorname{supp} u \subset K_{j}=\left\{\frac{1}{4} \leq|x| \leq 1\right\},\|u\|=1$, and $\left\|P_{j, \epsilon, \lambda} u\right\| \leq 2 C_{j}(\epsilon, \lambda)$. Proceeding as in iv), we easily find

$$
\left\|P_{j, \epsilon, \lambda} u\right\| \geq 2^{2 j-4}, \quad \text { and } \quad\left\|P_{j, \epsilon, \lambda} u\right\| \geq \frac{\inf _{x \in K_{j}}\left|g_{j}(x)\right|}{\epsilon 2^{k j}},
$$

where $g_{j}(x)=2^{k j} f\left(2^{j} x\right)-2^{k j} \epsilon \lambda$. If $2^{j} \geq \epsilon^{-\frac{1}{k+4}}$, the first inequality already implies (4.21). In the converse case, we integrate by parts and obtain the relation

$$
\left\|P_{j, \epsilon, \lambda} u\right\|^{2}+2\|u\|^{2}=\left\|Q_{j, \epsilon, \lambda} u\right\|^{2}+2\left\|x u_{x}\right\|^{2}+2^{4 j}\left\|x^{2} u\right\|^{2} \geq\left\|Q_{j, \epsilon, \lambda} u\right\|^{2},
$$

where $Q_{j, \epsilon, \lambda}=P_{j, \epsilon, \lambda}-2^{2 j} x^{2}$. Thus $\left\|P_{j, \epsilon, \lambda} u\right\| \geq\left\|Q_{j, \epsilon, \lambda} u\right\|-\sqrt{2}\|u\|$, and combining this estimate with (4.22) we obtain the sharper result

$$
2 C_{j}(\epsilon, \lambda) \geq\left\|P_{j, \epsilon, \lambda} u\right\| \geq \frac{1}{3}\left(2^{2 j-4}+\frac{\inf _{x \in K_{j}}\left|g_{j}(x)\right|}{\epsilon 2^{k j}}+\left\|Q_{j, \epsilon, \lambda} u\right\|-\sqrt{2}\right) .
$$


We next observe that

$$
Q_{j, \epsilon, \lambda}=\frac{1}{\epsilon 2^{k j}}\left(-h^{2} \partial_{x}^{2}+i g_{j}\right), \quad \text { where } \quad h^{2}=\epsilon 2^{(k-2) j}=\mathcal{O}\left(\epsilon^{\frac{6}{k+4}}\right) .
$$

From Hypothesis 1.6, we know that the derivative $g_{j}^{\prime}(x)=2^{(k+1) j} f^{\prime}\left(2^{j} x\right)$ converges to $-k x|x|^{-k}$ as $j \rightarrow \infty$, uniformly on $K_{j}$. In particular, there exists $C_{3}>0$ such that $\left|g_{j}^{\prime}(x)\right| \geq C_{3}$ and $\left|g_{j}^{\prime \prime}(x)\right| \leq C_{3}^{-1}$ for all $x \in K_{j}$ if $j$ is sufficiently large. Thus, using a partition of unity and a Taylor expansion like in case ii), we can again reduce the analysis to the microlocal model (4.16). We obtain the following lower bound

$$
\left\|\left(-h^{2} \partial_{x}^{2}+i g_{j}\right) u\right\| \geq C_{4} h^{2 / 3}\|u\|, \quad \text { for all } u \in C_{0}^{\infty}(\mathbb{R}) \text { with } \operatorname{supp} u \subset K_{j} .
$$

Returning to (4.24), we arrive at the estimate

$$
2 C_{j}(\epsilon, \lambda) \geq \frac{1}{3}\left(2^{2 j-4}+\frac{C_{4} \epsilon^{1 / 3} 2^{(k-2) j / 3}}{\epsilon 2^{k j}}-\sqrt{2}\right) \geq C_{5} \epsilon^{-\frac{2}{k+4}},
$$

which proves (4.21).

Assume now that there is a family $(\epsilon, \lambda)$ with $\lambda=\lambda(\epsilon)$ such that $\epsilon \rightarrow 0$ and $\kappa(\epsilon, \lambda) \geq C_{6} \epsilon^{\frac{2}{k+4}}$ for some $C_{6}>0$. By Lemma 4.4, there exists $C_{7}>0$ such that, for all $(\epsilon, \lambda)$,

$$
C_{j}(\epsilon, \lambda) \leq C_{7} \epsilon^{-\frac{2}{k+4}}, \quad \text { for some } j=j(\epsilon, \lambda) \in \mathbb{N} .
$$

It is clear that $j(\epsilon, \lambda) \rightarrow \infty$ as $\epsilon \rightarrow 0$, because if $j$ stays in a finite set $\{0, \ldots, J\}$ we have the lower bound $C_{j}(\epsilon, \lambda) \geq C_{J} \epsilon^{-1 / 2}$ which is incompatible with (4.26). We also know that $2^{j} \leq C \epsilon^{-1 /(k+4)}$, otherwise (4.24) would contradict (4.26) if $\epsilon$ is small. Thus we can use estimate (4.25) which implies, in view of (4.26),

$$
C_{8}^{-1} \epsilon^{-\frac{1}{k+4}} \leq 2^{j} \leq C_{8} \epsilon^{-\frac{1}{k+4}}, \quad \text { for some } C_{8} \geq 1 .
$$

Next we choose $u$ as before and we deduce from (4.22), (4.26) that

$$
2 C_{7} \epsilon^{-\frac{2}{k+4}} \geq 2 C_{j}(\epsilon, \lambda) \geq\left\|P_{j, \epsilon, \lambda} u\right\| \geq \frac{\inf _{x \in K_{j}}\left|g_{j}(x)\right|}{\epsilon 2^{k j}} .
$$

As $g_{j}(x)=2^{k j} f\left(2^{j} x\right)-2^{k j} \epsilon \lambda$, this implies by direct calculation

$$
\frac{1}{\epsilon 2^{k j}} \inf _{x \in K_{j}} 2^{k j} f\left(2^{j} x\right)-2 C_{7} \epsilon^{-\frac{2}{k+4}} \leq \lambda \leq \frac{1}{\epsilon 2^{k j}} \sup _{x \in K_{j}} 2^{k j} f\left(2^{j} x\right)+2 C_{7} \epsilon^{-\frac{2}{k+4}} .
$$

Since $2^{k j} f\left(2^{j} x\right) \rightarrow|x|^{-k}$ uniformly in $K_{j}$ as $j \rightarrow \infty$ by Hypothesis [1.6, and since $2^{j}$ satisfies (4.27), it follows immediately from (4.28) that

$$
C_{9}^{-1} \epsilon^{-\frac{4}{k+4}} \leq \lambda \leq C_{9} \epsilon^{-\frac{4}{k+4}}
$$

for some $C_{9} \geq 1$, which is the desired estimate.

Finally, it remains to check the optimality of the various estimates established so far. This is a rather straightforward task, because the arguments we gave to bound $\kappa(\epsilon, \lambda)$ from above also indicate how to choose an appropriate test function $u \in C_{0}^{\infty}(\mathbb{R})$ which "saturates" the inequality and gives the corresponding lower bound. We shall provide the details only in the last case $\mathbf{v}$ ), because this implies the upper bound on $\Psi(\epsilon)$ in Theorem 1.8 . 
Fix $x_{0} \in(1 / 4,1)$ and take $j \in \mathbb{N}$ very large. We define $\epsilon>0, \lambda>0$, and $h>0$ by the following relations

$$
2^{j}=\epsilon^{-\frac{1}{k+4}}, \quad h^{2}=\epsilon 2^{(k-2) j}, \quad \epsilon \lambda=f\left(2^{j} x_{0}\right) .
$$

Next, we choose $v \in C_{0}^{\infty}(\mathbb{R})$ such that $\|v\|=1$ and $\operatorname{supp} v \subset[-1,1]$, and we denote

$$
u_{h}(x)=\frac{1}{h^{1 / 3}} v\left(\frac{x-x_{0}}{h^{2 / 3}}\right), \quad x \in \mathbb{R} .
$$

It is clear that $u_{h} \in C_{0}^{\infty}(\mathbb{R}),\left\|u_{h}\right\|=1$, and that $\operatorname{supp} u_{h} \subset K_{j}$ if $h>0$ is sufficiently small, that is, if $j$ is sufficiently large. Recalling that

$$
P_{j, \epsilon, \lambda}=\frac{1}{\epsilon 2^{k j}}\left(-h^{2} \partial_{x}^{2}+h^{2 / 3} x^{2}+i g_{j}(x)\right), \quad \text { where } \quad g_{j}(x)=2^{k j} f\left(2^{j} x\right)-2^{k j} \epsilon \lambda,
$$

we claim that there exists $C_{0}>0$ independent of $j$ (hence of $\epsilon, \lambda$ ) such that

$$
\left\|P_{j, \epsilon, \lambda} u_{h}\right\| \leq C_{0} \frac{h^{2 / 3}}{\epsilon 2^{k j}}=C_{0} \epsilon^{-\frac{2}{k+4}} .
$$

This implies that $C_{j}(\epsilon, \lambda) \leq C_{0} \epsilon^{-\frac{2}{k+4}}$, hence $\kappa(\epsilon, \lambda) \geq C_{0}^{-1} \epsilon^{\frac{2}{k+4}}$ by (4.8).

Checking (4.31) is of course straightforward. First, using (4.30), we find $\left\|h^{2} \partial_{x}^{2} u_{h}\right\|=$ $h^{2 / 3}\left\|v^{\prime \prime}\right\|$. Next, since $x^{2} \leq x_{0}^{2}+2\left|x-x_{0}\right|$ for all $x \in K_{j}$, we have $\left\|x^{2} u_{h}\right\| \leq x_{0}^{2}\|v\|+2 h^{2 / 3}\|v\| \leq C$. Finally, since $g_{j}\left(x_{0}\right)=0$ by our choice of $\lambda$, we have for all $x \in[1 / 4,1]$

$$
\left|g_{j}(x)\right| \leq\left|x-x_{0}\right| \sup _{z \in[1 / 4,1]}\left|g_{j}^{\prime}(z)\right| \leq C\left|x-x_{0}\right|
$$

where the constant $C$ does not depend on $j$ by Hypothesis [1.6. Thus $\left\|g_{j} u_{h}\right\| \leq C h^{2 / 3}\|v\|$, and the proof of (4.31) is complete.

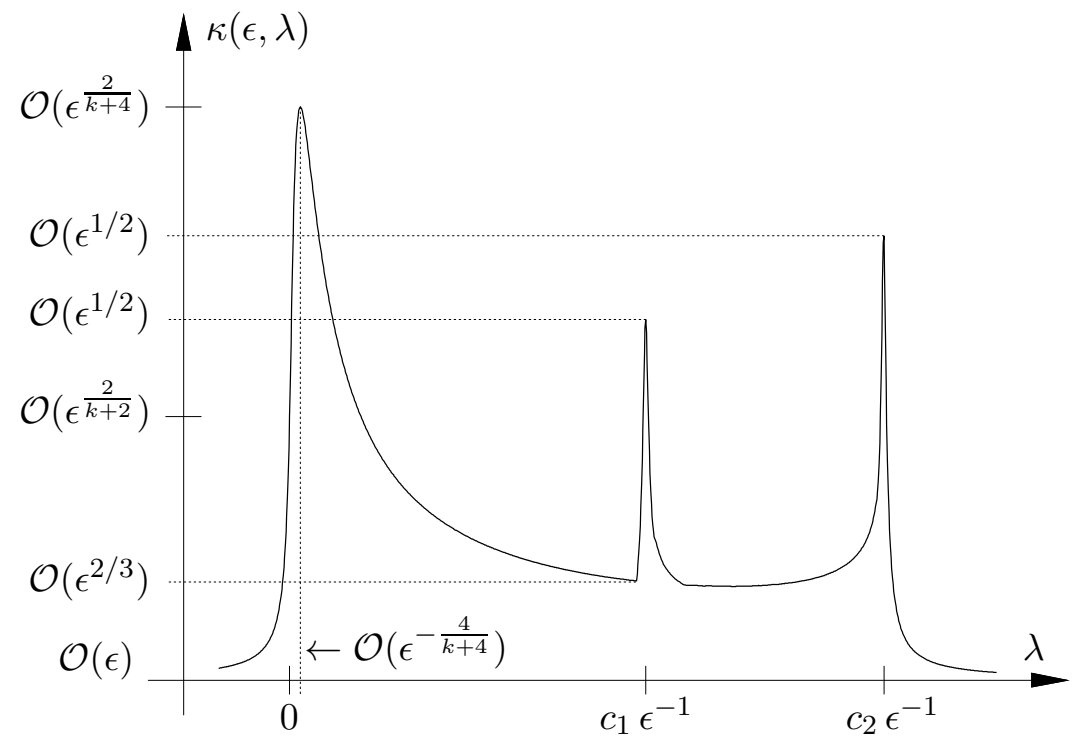

Fig. 3: The resolvent $\kappa(\epsilon, \lambda)=\left\|\left(H_{\epsilon}-i \lambda\right)^{-1}\right\|$ is represented for $\epsilon=2^{-14}$ and $f$ given by (4.32). 
To illustrate the various regimes appearing in Proposition 4.1, we computed numerically the function $\kappa(\epsilon, \lambda)$ in the particular case when

$$
f(x)=\frac{1+3 x^{2}}{\left(1+x^{2} / 3\right)^{3}}, \quad x \in \mathbb{R} .
$$

Up to a multiplicative factor, this function satifies Hypothesis 1.6, with $k=4$. It has exactly two critical values $c_{1}=1, c_{2}=27 / 16$, which correspond to the critical points $x_{1}=0$ and $x_{2}= \pm 1$ respectively. The result for $\epsilon=2^{-14}$ is displayed in Fig. 3. The large peak near the origin corresponds to the regime described in $\mathbf{v}$ ) above, while the two sharp spikes are associated to the critical values of $f$, as explained in iii). The predictions of i), ii) are also confirmed, but the value $\epsilon=2^{-14}$ is not small enough in this example to test the prediction of iv): we know from Proposition 4.1 that $\kappa(\epsilon, 0)=\mathcal{O}\left(\epsilon^{1 / 3}\right)$ since $k=4$ and $0 \notin f(\mathbb{R})$, but in Fig. 3 the value computed for $\lambda=0$ is still clearly below the $\mathcal{O}\left(\epsilon^{1 / 2}\right)$ spikes associated to the critical values of $f$.

\section{Spectral lower bounds}

This section is devoted to the detailed study of the particular example (1.16). We shall first prove Proposition 1.9, and then illustrate the optimality of the lower bound (1.17) using numerical calculations and heuristic semiclassical arguments. As was mentioned in the introduction, the estimate (1.17) implies that $\left\|e^{-t H_{\epsilon}}\right\| \leq C e^{-\mu_{\epsilon} t}$ for some $C>0$ if $\mu_{\epsilon}=\frac{1}{2} M_{5} \epsilon^{-\nu^{\prime}}$, but it follows from Lemma 1.1 that $C \geq e^{-1} \exp \left(\mu_{\epsilon} / \Psi(\epsilon)\right)$ where $\mu_{\epsilon} / \Psi(\epsilon)=\mathcal{O}\left(\epsilon^{\bar{\nu}-\nu^{\prime}}\right) \rightarrow \infty$ as $\epsilon \rightarrow 0$. It is very likely that such an exponential bound in the semiclassical framework cannot be obtained while remaining in the $C^{\infty}$ category. Thus, like in the study of resonances (see [1, 2, 12, 15]), we shall exploit the analyticity properties of the function (1.16) by performing a complex deformation. In this example we shall observe an interesting phenomenon when comparing the pseudospectral and spectral estimates. In contrast to what happens for resonances, the large peak of the quantity $\kappa(\epsilon, \lambda)=\left\|\left(H_{\epsilon}-i \lambda\right)^{-1}\right\|$ in the region $\lambda \sim \epsilon^{-4 /(k+4)}$ (see Fig. 3) is not due to the presence of an eigenvalue nearby, for the deformed operator.

Proof of Proposition 1.9: We shall prove the lower bound (1.17) in two steps. We first observe that, in view of Proposition 4.1, it is sufficient to show that there exist positive constants $c_{1}, c_{2}$ such that

$$
\sigma\left(H_{\epsilon}\right) \cap\left\{z \in \mathbb{C} ; c_{1} \operatorname{Re} z \leq|\operatorname{Im} z| \leq \frac{c_{2}}{\epsilon}\right\}=\emptyset,
$$

for $\epsilon>0$ small enough. Indeed, assume for the moment that (5.1) holds. If $\lambda \in \mathbb{R}$ is such that $|\lambda| \geq c_{2} \epsilon^{-1}$, the proof of Proposition 4.1 shows that $\kappa(\epsilon, \lambda)=\left\|\left(H_{\epsilon}-i \lambda\right)^{-1}\right\| \leq C \epsilon^{1 / 2}$ for some $C>0$, hence any $z \in \sigma\left(H_{\epsilon}\right)$ with $|\operatorname{Im} z| \geq c_{2} \epsilon^{-1}$ necessarily satisfies $\operatorname{Re} z \geq C^{-1} \epsilon^{-1 / 2}$. On the other hand, the spectrum of $H_{\epsilon}$ in the strip $|\operatorname{Im} z| \leq c_{2} \epsilon^{-1}$ is concentrated in the sector $|\operatorname{Im} z| \leq c_{1} \operatorname{Re} z$ by (5.1), hence to prove the lower bound (1.17) it is sufficient to consider the smaller strip $|\operatorname{Im} z| \leq \epsilon^{-\nu^{\prime}}$. We thus take $\lambda \in \mathbb{R}$ with $|\lambda| \leq \epsilon^{-\nu^{\prime}}$ and we estimate $\kappa(\epsilon, \lambda)$ as in the proof of Proposition 4.1. Using exactly the same notations, we find for all $j \in \mathbb{N}$ :

$$
C_{j}(\epsilon, \lambda) \geq \frac{1}{2}\left(2^{2 j-4}+\frac{1}{\epsilon 2^{k j}} \inf _{x \in K_{j}}\left|2^{k j} f\left(2^{j} x\right)-2^{k j} \epsilon \lambda\right|\right),
$$

see e.g. (4.22). As usual it is sufficient to consider large values of $j$, in which case $2^{k j} f\left(2^{j} x\right) \approx$ $|x|^{-k}$ for all $x \in K_{j}=\left\{x \in \mathbb{R} ; \frac{1}{4} \leq|x| \leq 1\right\}$. If $2^{k j} \epsilon|\lambda| \leq 1 / 4$, the infimum in (5.2) is larger than $1 / 2$ for large $j$, hence

$$
C_{j}(\epsilon, \lambda) \geq \frac{1}{2}\left(2^{2 j-4}+\frac{1}{\epsilon 2^{k j+1}}\right) \geq C \epsilon^{-\frac{2}{k+2}} \geq C \epsilon^{-\nu^{\prime}} .
$$


If $2^{k j} \epsilon|\lambda| \geq 1 / 4$ and $|\lambda| \leq \epsilon^{-\nu^{\prime}}$, then $2^{k j} \geq C \epsilon^{\nu^{\prime}-1}$ hence $C_{j}(\epsilon, \lambda) \geq 2^{2 j-5} \geq C \epsilon^{-\nu^{\prime}}$. Thus we have shown that $\kappa(\epsilon, \lambda)^{-1} \geq C \inf _{j} C_{j}(\epsilon, \lambda) \geq C \epsilon^{-\nu^{\prime}}$ if $|\lambda| \leq \epsilon^{-\nu^{\prime}}$, from which we deduce that any $z \in \sigma\left(H_{\epsilon}\right)$ with $|\operatorname{Im} z| \leq \epsilon^{-\nu^{\prime}}$ necessarily satisfies $\operatorname{Re} z \geq C^{-1} \epsilon^{-\nu^{\prime}}$. Summarizing, we have shown that there exists $M_{5}>0$ such that $H_{\epsilon}$ has no spectrum in the half-plane $\left\{\operatorname{Re} z \leq M_{5} \epsilon^{-\nu^{\prime}}\right\}$ if $\epsilon$ is sufficiently small, which is the desired result.

It remains to prove (5.1). Clearly this cannot be done using only the pseudospectral estimates of Proposition 4.1, because by Corollary 4.2 the domain $\left\{z \in \mathbb{C} ; c_{1} \operatorname{Re} z \leq|\operatorname{Im} z| \leq c_{2} \epsilon^{-1}\right\}$ meets the pseudospectrum of $H_{\epsilon}$ as $\epsilon \rightarrow 0$. Instead we shall implement a complex deformation method using the group of dilations $\left(U_{\theta} \varphi\right)(x)=e^{\theta / 2} \varphi\left(e^{\theta} x\right)$, which are unitary operators when $\theta \in \mathbb{R}$. If $f$ is given by (1.16), the multiplication operator by $(i / \epsilon) f(x)$ is a dilation analytic perturbation of the harmonic oscillator hamiltonian $H_{\infty}=-\partial_{x}^{2}+x^{2}$, according to Definition 8.1 in [2]. This implies that

$$
H_{\epsilon}(\theta)=U_{\theta} H_{\epsilon} U_{\theta}^{-1}=-e^{-2 \theta} \partial_{x}^{2}+e^{2 \theta} x^{2}+\frac{i}{\epsilon\left(1+e^{2 \theta} x^{2}\right)^{k / 2}}
$$

defines an analytic family of type (A) in the strip $S=\{\theta \in \mathbb{C} ;|\operatorname{Im} \theta|<\pi / 4\}$ (see [17]), with common domain $\mathcal{D}=D\left(H_{\infty}\right)$. In particular the spectrum of $H_{\epsilon}(\theta)$, which is always discrete, does not depend on $\theta \in S$.

We choose $\theta=i t_{k}$, where $t_{k}=\frac{\pi}{4(k+2)}$, and we first observe that the operator $H_{\epsilon}\left(i t_{k}\right)$ is still maximal accretive. Indeed, for all $(x, \xi) \in \mathbb{R}^{2}$,

$$
\begin{gathered}
e^{-2 i t_{k}} \xi^{2}+e^{2 i t_{k}} x^{2} \in\left\{z \in \mathbb{C} ;-2 t_{k} \leq \arg (z) \leq 2 t_{k}\right\} \subset\{z \in \mathbb{C} ; \operatorname{Re}(z) \geq 0\}, \\
\frac{i}{\epsilon} \frac{\left(1+e^{-2 i t_{k}} x^{2}\right)^{k / 2}}{\left|1+e^{2 i t_{k}} x^{2}\right|^{k}} \in\left\{z \in \mathbb{C} ; \frac{\pi}{2}-k t_{k} \leq \arg (z) \leq \frac{\pi}{2}\right\} \subset\{z \in \mathbb{C} ; \operatorname{Re}(z) \geq 0\},
\end{gathered}
$$

hence $\operatorname{Re}\left\langle H\left(i t_{k}\right) \varphi, \varphi\right\rangle \geq 0$ for all $\varphi \in \mathcal{D}$. It is therefore sufficient to reproduce part of the analysis done in Proposition 4.1 to estimate $\left\|\left(H_{\epsilon}-i \lambda\right)^{-1}\right\|$ for $\lambda \in \mathbb{R}$. Consider a partition of unity $\chi_{0}^{2}+\chi_{\infty}^{2}=1$ with $\operatorname{supp} \chi_{0} \subset(-1,1)$ and $\chi_{0} \equiv 1$ on $[-1 / 2,1 / 2]$. Applying the IMS localization formula (4.4) to the operator $Q=e^{2 i t_{k}}\left(H_{\epsilon}\left(i t_{k}\right)-i \lambda\right)$, and using the fact that

$$
\left\|\partial_{x} u\right\|^{2} \leq \frac{1}{\cos \left(2 t_{k}\right)} \operatorname{Re}\left\langle\left(H_{\epsilon}\left(i t_{k}\right)-i \lambda\right) u, u\right\rangle \leq \sqrt{2}\|u\|\left\|\left(H_{\epsilon}\left(i t_{k}\right)-i \lambda\right) u\right\|,
$$

we obtain

$$
3\left\|\left(H_{\epsilon}\left(i t_{k}\right)-i \lambda\right) u\right\|^{2}+C\|u\|^{2} \geq\left\|\left(H_{\epsilon}\left(i t_{k}\right)-i \lambda\right) \chi_{0} u\right\|^{2}+\left\|\left(H_{\epsilon}\left(i t_{k}\right)-i \lambda\right) \chi_{\infty} u\right\|^{2} .
$$

We need to estimate both terms in the right-hand side.

i) The localization of $u_{0}=\chi_{0} u$ in the interval $(-1,1)$ implies

$$
\begin{aligned}
\left\|u_{0}\right\| \|\left(H_{\epsilon}\left(i t_{k}\right)\right. & -i \lambda) u_{0} \| \geq \operatorname{Im}\left\langle\left(H_{\epsilon}\left(i t_{k}\right)-i \lambda\right) u_{0}, u_{0}\right\rangle \\
& \geq \frac{1}{\epsilon} \operatorname{Re}\left\langle\left(f\left(e^{i t_{k}} x\right)-\lambda \epsilon\right) u_{0}, u_{0}\right\rangle-\sin \left(2 t_{k}\right)\left\|\partial_{x} u_{0}\right\|^{2} \\
& \geq \frac{1}{\epsilon} \inf _{|x| \leq 1}\left(\operatorname{Re} f\left(e^{i t_{k}} x\right)-\epsilon \lambda\right)\left\|u_{0}\right\|^{2}-\tan \left(2 t_{k}\right)\left\|u_{0}\right\|\left\|\left(H_{\epsilon}\left(i t_{k}\right)-i \lambda\right) u_{0}\right\| .
\end{aligned}
$$

Since $\left|1+e^{2 i t_{k}} x^{2}\right| \leq 2$ when $|x| \leq 1$ and $-k t_{k} \leq \arg \left(f\left(e^{i t_{k}} x\right)\right) \leq 0$, we have

$$
\operatorname{Re} f\left(e^{i t_{k}} x\right) \geq \frac{\cos \left(k t_{k}\right)}{2^{k / 2}} \geq \frac{1}{2^{\frac{k+1}{2}}}, \quad \text { for }|x| \leq 1 .
$$


If we assume that $\epsilon|\lambda| \leq c_{2} \stackrel{\text { def }}{=} 2^{-\frac{k+2}{2}}$, we thus arrive at the lower bound

$$
2\left\|\left(H_{\epsilon}\left(i t_{k}\right)-i \lambda\right) \chi_{0} u\right\| \geq\left(1+\tan \left(2 t_{k}\right)\right)\left\|\left(H_{\epsilon}\left(i t_{k}\right)-i \lambda\right) \chi_{0} u\right\| \geq c \epsilon^{-1}\left\|\chi_{0} u\right\|,
$$

where $c=2^{-\frac{k+1}{2}}-2^{-\frac{k+2}{2}}>0$.

ii) On the other hand, setting $u_{\infty}=\chi_{\infty} u$ and assuming that $u_{\infty} \neq 0$, we observe that

$$
\frac{\left\|\left(H_{\epsilon}\left(i t_{k}\right)-i \lambda\right) u_{\infty}\right\|}{\left\|u_{\infty}\right\|} \geq\left|\frac{\left\langle H_{\epsilon}\left(i t_{k}\right) u_{\infty}, u_{\infty}\right\rangle}{\left\|u_{\infty}\right\|^{2}}-i \lambda\right| \geq \inf _{z \in S_{k}}|z-i \lambda|
$$

where $S_{k}$ is any sector in the complex plane that is large enough to contain the quantity

$$
\left\langle H_{\epsilon}\left(i t_{k}\right) u_{\infty}, u_{\infty}\right\rangle=e^{-2 i t_{k}}\left\|\partial_{x} u_{\infty}\right\|^{2}+e^{2 i t_{k}}\left\|x u_{\infty}\right\|^{2}+\frac{e^{i\left(\pi / 2-k t_{k}\right)}}{\epsilon}\left\langle\frac{\left(e^{2 i t_{k}}+x^{2}\right)^{k / 2}}{\left|1+e^{2 i t_{k}} x^{2}\right|^{k}} u_{\infty}, u_{\infty}\right\rangle
$$

for any $u_{\infty} \in \mathcal{D}$ with $\operatorname{supp} u_{\infty} \subset \mathbb{R} \backslash\left[-\frac{1}{2}, \frac{1}{2}\right]$. If we denote

$$
\delta_{k}=\frac{1}{2} \arg \left(e^{2 i t_{k}}+\frac{1}{4}\right) \in\left(0, t_{k}\right), \quad \text { so that } \quad k \delta_{k} \geq \max _{|x| \geq 1 / 2} \arg \left(\left(e^{2 i t_{k}}+x^{2}\right)^{k / 2}\right),
$$

we have $\frac{\pi}{2}>\frac{\pi}{2}-k\left(t_{k}-\delta_{k}\right)>\frac{\pi}{2}-k t_{k}>2 t_{k}$, hence we can choose

$$
S_{k}=\left\{z \in \mathbb{C} ;-2 t_{k} \leq \arg z \leq \frac{\pi}{2}-k\left(t_{k}-\delta_{k}\right)\right\},
$$

and there exists $c_{k}>0$ such that $\inf _{z \in S_{k}}|z-i \lambda| \geq c_{k}|\lambda|$.

If we now combine the lower bounds of both terms in the right-hand side of (5.3), and if we use in addition the fact that $\left\|\left(H_{\epsilon}\left(i t_{k}\right)-i \lambda\right) u\right\| \geq \cos \left(2 t_{k}\right)\|u\|$, we conclude that there exists $c_{1}>0$ such that

$$
\left\|\left(H_{\epsilon}\left(i t_{k}\right)-i \lambda\right) u\right\| \geq C \min \left\{\frac{c}{2 \epsilon}, c_{k}|\lambda|\right\}\|u\| \geq \frac{2|\lambda|}{c_{1}}\|u\|, \quad \text { for all } u \in \mathcal{D},
$$

when $\lambda \in \mathbb{R}$ satisfies $|\lambda| \leq c_{2} \epsilon^{-1}$. Thus the deformed operator $H_{\epsilon}\left(i t_{k}\right)$ is sectorial in a neighborhood of the origin in the complex plane, uniformly for all $\epsilon \in(0,1]$. In particular, if $z=\mu+i \lambda$ with $0<c_{1} \mu \leq|\lambda| \leq c_{2} \epsilon^{-1}$, it follows from (5.4) that

$$
\left\|\left(H_{\epsilon}\left(i t_{k}\right)-z\right)^{-1}\right\| \leq \frac{\left\|\left(H_{\epsilon}\left(i t_{k}\right)-i \lambda\right)^{-1}\right\|}{1-\mu\left\|\left(H_{\epsilon}\left(i t_{k}\right)-i \lambda\right)^{-1}\right\|} \leq \frac{\frac{1}{2} c_{1}|\lambda|^{-1}}{1-\mu\left(\frac{1}{2} c_{1}|\lambda|^{-1}\right)} \leq \frac{c_{1}}{|\lambda|},
$$

hence $z \notin \sigma\left(H_{\epsilon}\left(i t_{k}\right)\right) \equiv \sigma\left(H_{\epsilon}\right)$. This proves (5.1) , since we already know that $H_{\epsilon}$ has no spectrum in the half-plane $\operatorname{Re} z \leq 0$.

Although Propositions 4.1 and 1.9 provide the necessary information for comparing the spectral bound $\Sigma(\epsilon)$ and the pseudospectral quantity $\Psi(\epsilon)$, the analysis can be pushed further in the particular example (1.16). As it is the case for $\Psi(\epsilon)$, the actual behavior of $\Sigma(\epsilon)$ as $\epsilon \rightarrow 0$ results from a competition between contributions due to the (unique) critical point of $f$, and to the behavior of $f(x)$ as $|x| \rightarrow \infty$. Instead of giving a complete proof, we briefly sketch the main idea, which is adapted from the usual semiclassical techniques of harmonic approximation (see for example [9]) and complex deformation (see for example [18, 21]). We also verify our conclusions using numerical simulations.

We first write $\epsilon H_{\epsilon}=-h^{2} \partial_{x}^{2}+V\left(x, h^{2}\right)$ where $h=\epsilon^{1 / 2}$ and $V\left(x, h^{2}\right)=h^{2} x^{2}+i f(x)$, and we remark that $\epsilon H_{\epsilon}$ is not exactly a semiclassical operator, because the potential $V$ depends 
on $h$ in a nontrivial way which makes the comparison with quadratic approximations at critical points more difficult. In the sector $\{z \in \mathbb{C} ;|\arg z| \leq \pi / 4\}$, the function $V(z, \epsilon)$ has exactly three critical points: $z=0$, and $z= \pm z_{\epsilon}$, where

$$
z_{\epsilon}=\left\{\left(\frac{i k}{2 \epsilon}\right)^{2 \nu}-1\right\}^{1 / 2}=\left(\frac{i k}{2 \epsilon}\right)^{\nu}+\mathcal{O}\left(\epsilon^{\nu}\right), \quad \text { where } \quad \nu=\frac{1}{k+2} .
$$

The quadratic approximations of $H_{\epsilon}$ are respectively:

i) At $z=0: H_{\epsilon}^{0}=-\partial_{x}^{2}+\frac{i f(0)}{\epsilon}+\left(1+\frac{i f^{\prime \prime}(0)}{2 \epsilon}\right) x^{2}$. The eigenvalues of $H_{\epsilon}^{0}$ are

$$
\mu_{n}^{0}(\epsilon)=\frac{i}{\epsilon}+(2 n+1) \omega_{\epsilon}, \quad n \in \mathbb{N}, \quad \text { where } \quad \omega_{\epsilon}=\left(1+\frac{i}{2 \epsilon} f^{\prime \prime}(0)\right)^{1 / 2} .
$$

ii) At $z= \pm z_{\epsilon}: H_{\epsilon}^{ \pm}=-\partial_{x}^{2}+D_{\epsilon}+\Omega_{\epsilon}^{2} x^{2}$, with

$$
\begin{aligned}
& D_{\epsilon}=z_{\epsilon}^{2}+\frac{i}{\epsilon} f\left(z_{\epsilon}\right)=\frac{k+2}{k} z_{\epsilon}^{2}+\frac{2}{k}=\frac{k+2}{k}\left(\frac{i k}{2 \epsilon}\right)^{2 \nu}-1, \\
& \Omega_{\epsilon}^{2}=1+\frac{i}{2 \epsilon} f^{\prime \prime}\left(z_{\epsilon}\right)=(k+2) \frac{z_{\epsilon}^{2}}{1+z_{\epsilon}^{2}}=(k+2)\left(1-\left(\frac{2 \epsilon}{i k}\right)^{2 \nu}\right) .
\end{aligned}
$$

The eigenvalues of $H_{\epsilon}^{ \pm}$are $\nu_{n}^{0}(\epsilon)=D_{\epsilon}+(2 n+1) \Omega_{\epsilon}, n \in \mathbb{N}$.

After a complex rotation, these quadratic approximations are transformed into real harmonic oscillators $\mathbf{H}_{\epsilon}^{0}$ and $\mathbf{H}_{\epsilon}^{ \pm}$, respectively. The actual complex deformation has to be chosen carefully so that the non-self-adjoint corrections to $\mathbf{H}_{\epsilon}^{0}\left(\operatorname{resp} . \mathbf{H}_{\epsilon}^{ \pm}\right)$still allow for a good control of the resolvent norm of the deformed hamiltonian $\mathbf{H}_{\epsilon}$ when $z$ is close to $\mu_{n}^{0}(\epsilon)\left(\operatorname{resp} . \nu_{n}^{0}(\epsilon)\right)$. This requires an accurate analysis, which is left for a future work. In any case, on the basis of the arguments above, we expect that the full operator $H_{\epsilon}$ has two sets of eigenvalues $\left\{\mu_{n}(\epsilon)\right\}$ and $\left\{\nu_{n}(\epsilon)\right\}$ which satisfy:

$$
\begin{aligned}
& \mu_{n}(\epsilon)=\frac{i}{\epsilon}+(2 n+1) \omega_{\epsilon}+\mathcal{O}(1), \quad n=0,1,2, \ldots \\
& \nu_{n}(\epsilon)=D_{\epsilon}+(2 n+1) \Omega_{\epsilon}+\mathcal{O}\left(\epsilon^{\nu}\right), \quad n=0,1,2, \ldots
\end{aligned}
$$

This leads to the following conjecture:

Conjecture 5.1 Fix $k>0$ and let $f: \mathbb{R} \rightarrow \mathbb{R}$ be as in (1.16). Then the spectral bound of $H_{\epsilon}$ satisfies, when $\epsilon>0$ is small,

$$
\Sigma(\epsilon)=\min \left\{\operatorname{Re}\left(\mu_{0}(\epsilon)\right), \operatorname{Re}\left(\nu_{0}(\epsilon)\right)\right\},
$$

where $\mu_{0}(\epsilon)$ is given by (5.5) and $\nu_{0}(\epsilon)$ by (5.6).

Conjecture 5.1 predicts the following asymptotic expansions as $\epsilon \rightarrow 0$ :

$$
\Sigma(\epsilon)= \begin{cases}\frac{1}{2}\left(\frac{k}{\epsilon}\right)^{1 / 2}+\mathcal{O}(1), & \text { if } \quad k \leq 2 \\ \frac{k+2}{k}\left(\frac{k}{2 \epsilon}\right)^{2 \nu} \cos (\pi \nu)+\sqrt{k+2}-1+\mathcal{O}\left(\epsilon^{\nu}\right) & \text { if } \quad k>2\end{cases}
$$

with $\nu=(k+2)^{-1}$, in full agreement with Proposition 1.9. 
To test these predictions, we have computed an approximation of $\Sigma(\epsilon)$ using a standard finite difference scheme, for three different choices of $k$ and for values of $\epsilon$ ranging from $2^{-1}$ to $2^{-18}$. Calculations were made on a large interval $[-L, L]$ with Dirichlet boundary conditions, using at least 500 grid points. The results are collected in Fig. 4, where the dots represent the values computed numerically and the solid lines the theoretical predictions, namely $\operatorname{Re}\left(\mu_{0}^{0}(\epsilon)\right)$ for $k \leq 2$ and $\operatorname{Re}\left(\nu_{0}^{0}(\epsilon)\right)$ for $k>2$. The agreement is excellent and leaves little doubt on the validity of Conjecture 5.1 .

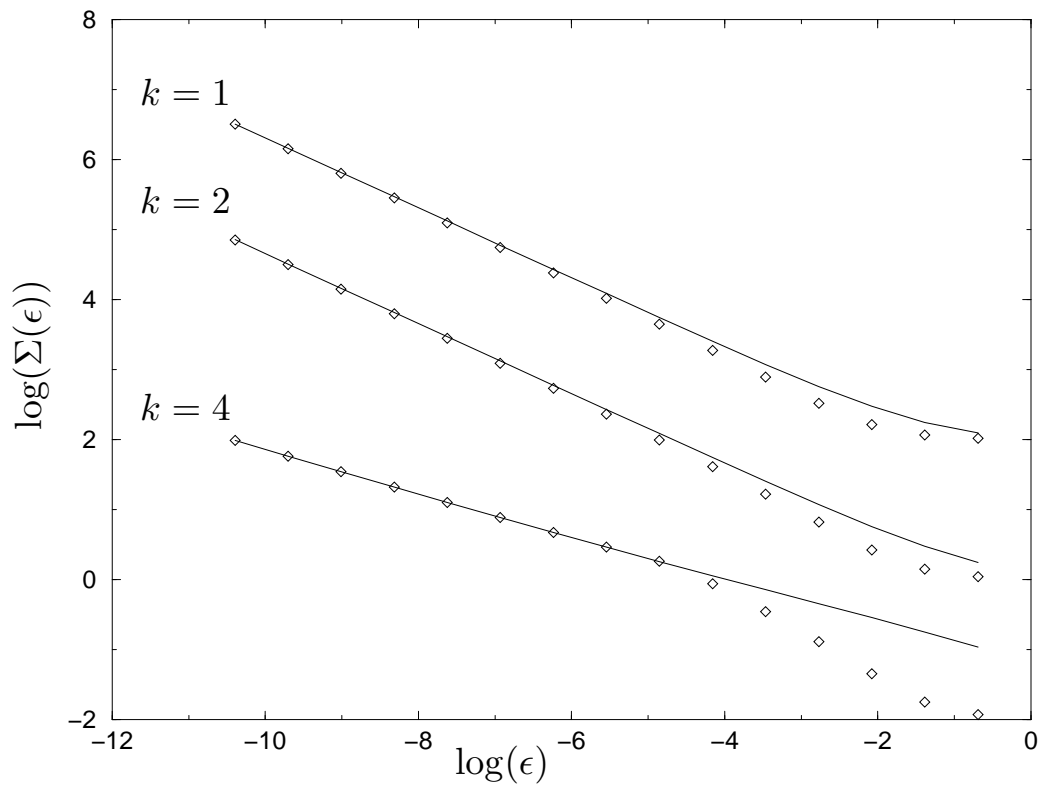

Fig. 4: The logarithm of $\Sigma(\epsilon)$ is represented as a function of $\log (\epsilon)$ for three choices of the parameter $k$. The dots correspond to the values computed numerically, and the solid lines to the theoretical predictions given by $\operatorname{Re}\left(\mu_{0}^{0}(\epsilon)\right)(k=1,2)$ and $\operatorname{Re}\left(\nu_{0}^{0}(\epsilon)\right)(k=4)$. To improve readability, the upper curve has been shifted up by two units and the lower curve shifted down by two units.

The heuristic arguments above give a correct prediction not only for $\Sigma(\epsilon)$, but also for all eigenvalues of $H_{\epsilon}$ with sufficiently low real part. Let us first consider the case when $k=4$ and $\epsilon=2^{-18}$. In Table 1 below, we have listed the five eigenvalues of $H_{\epsilon}$ with smallest real part, together with the approximate eigenvalues $\mu_{n}^{0}(\epsilon)$ and $\nu_{n}^{0}(\epsilon)$ for $n=0, \ldots, 4$. It is obvious that $\nu_{n}^{0}(\epsilon)$ is an excellent approximation of $\lambda_{n}(\epsilon)$.

\begin{tabular}{cccc}
\hline$n$ & $\lambda_{n}(\epsilon)$ & $\mu_{n}^{0}(\epsilon)$ & $\nu_{n}^{0}(\epsilon)$ \\
\hline 0 & $106.18+60.48 i$ & $512+2.616 \cdot 10^{5} i$ & $106.18+60.48 i$ \\
1 & $111.02+60.51 i$ & $1536+2.606 \cdot 10^{5} i$ & $111.06+60.50 i$ \\
2 & $115.83+60.54 i$ & $2560+2.596 \cdot 10^{5} i$ & $115.93+60.51 i$ \\
3 & $120.61+60.58 i$ & $3584+2.586 \cdot 10^{5} i$ & $120.80+60.53 i$ \\
4 & $125.36+60.63 i$ & $4608+2.575 \cdot 10^{5} i$ & $125.67+60.54 i$ \\
\hline
\end{tabular}

Table 1: The first few eigenvalues of $H_{\epsilon}$ for $k=4$ and $\epsilon=2^{-18}$, together with the predictions $\mu_{n}^{0}(\epsilon)$ and $\nu_{n}^{0}(\epsilon)$.

We next consider the case when $k=2$, which is especially interesting because both $\operatorname{Re}\left(\mu_{n}^{0}(\epsilon)\right)$ and $\operatorname{Re}\left(\nu_{n}^{0}(\epsilon)\right)$ grow with the same rate $\mathcal{O}\left(\epsilon^{-1 / 2}\right)$ as $\epsilon \rightarrow 0$. However we see from (5.5), (5.6) 
that $\operatorname{Re}\left(\mu_{n}^{0}(\epsilon)\right) \approx(2 n+1) /(2 \epsilon)^{1 / 2}$ while $\operatorname{Re}\left(\nu_{n}^{0}(\epsilon)\right) \approx(2 / \epsilon)^{1 / 2}+4 n+1$. It follows that the eigenvalue of $H_{\epsilon}$ with minimal real part is well approximated by $\mu_{0}^{0}(\epsilon)$, and thus corresponds to a semiclassical mode near the origin, while the subsequent eigenvalues are well approximated by $\nu_{n}^{0}(\epsilon)$ for $n=0,1,2, \ldots$ and therefore correspond to semiclassical modes near infinity. This prediction is clearly confirmed by the values listed in Table 2 below. It is also possible to verify that the eigenfunctions corresponding to $\lambda_{n}(\epsilon)$ are nicely approximated either by an eigenfunction of the quadratic approximation $H_{\epsilon}^{0}$ at $z=0$ (if $n=0$ ), or by an eigenfunction of the quadratic approximation $H_{\epsilon}^{ \pm}$at $z= \pm z_{\epsilon}$ (if $n=1,2, \ldots$ ).

\begin{tabular}{cccc}
\hline$n$ & $\lambda_{n}(\epsilon)$ & $\mu_{n}^{0}(\epsilon)$ & $\nu_{n}^{0}(\epsilon)$ \\
\hline 0 & $44.54+4051 i$ & $45.26+4050 i$ & $91.50+90.52 i$ \\
1 & $91.50+90.52 i$ & $135.78+3960 i$ & $95.48+90.54 i$ \\
2 & $95.47+90.54 i$ & $226.30+3869 i$ & $99.45+90.57 i$ \\
3 & $99.48+90.56 i$ & $316.82+3779 i$ & $103.43+90.59 i$ \\
4 & $103.2+90.58 i$ & $407.34+3688 i$ & $107.41+90.61 i$ \\
\hline
\end{tabular}

Table 2: The first few eigenvalues of $H_{\epsilon}$ for $k=2$ and $\epsilon=2^{-12}$, together with the predictions $\mu_{n}^{0}(\epsilon)$ and $\nu_{n}^{0}(\epsilon)$.

Finally we turn our attention to the case when $k=1$. Here we expect that the eigenvalues of $H_{\epsilon}$ with lowest real part are well approximated by $\mu_{n}^{0}(\epsilon)$, for $n=0,1,2, \ldots$ This is indeed the case, although the spectrum of $H_{\epsilon}$ seems more difficult to compute in this regime, due to numerical instabilities. Nevertheless, for $\epsilon=2^{-12}$, we were able to check that at least the first three eigenvalues of $H_{\epsilon}$ are correctly approximated by $\mu_{n}^{0}(\epsilon)$, see Table 3 below. The errors are larger than in the preceding cases, but we should take into account the fact that the remainder term in (5.5) is $\mathcal{O}(1)$.

\begin{tabular}{cccc}
\hline$n$ & $\lambda_{n}(\epsilon)$ & $\mu_{n}^{0}(\epsilon)$ & $\nu_{n}^{0}(\epsilon)$ \\
\hline 0 & $31.46+4064 i$ & $32.00+4064 i$ & $242.63+419.00 i$ \\
1 & $93.34+4002 i$ & $96.02+4000 i$ & $246.09+419.01 i$ \\
2 & $153.2+3940 i$ & $160.0+3936 i$ & $249.55+419.01 i$ \\
\hline
\end{tabular}

Table 3: The first few eigenvalues of $H_{\epsilon}$ for $k=1$ and $\epsilon=2^{-12}$, together with the predictions $\mu_{n}^{0}(\epsilon)$ and $\nu_{n}^{0}(\epsilon)$.

\section{A Appendix}

This appendix gathers the proofs of Lemmas 1.1, 1.3, 1.7, and provides a variant of Lemma 1.7 which is needed in Section 3.3 .

We first give a proof Lemma 1.1, which relates the quantities $\Sigma$ and $\Psi$ to the norm of the semigroup $e^{-t A}$ for a sectorial operator, as an application of the Laplace transformation.

Proof of Lemma 1.1. Let $A$ be a maximal accretive operator in a Hilbert space $X$, with numerical range $\Theta(A)$ contained in the sector $\Delta_{\alpha}=\left\{z \in \mathbb{C} ;|\arg z| \leq \frac{\pi}{2}-2 \alpha\right\}$ for some $\alpha \in\left(0, \frac{\pi}{4}\right]$.

i) Assume first that $\left\|e^{-t A}\right\| \leq C e^{-\mu t}$ for all $t \geq 0$. Then the resolvent

$$
(A-z)^{-1}=\int_{0}^{\infty} e^{-t A} e^{t z} \mathrm{t}
$$


is defined (at least) for all $z \in \mathbb{C}$ with $\operatorname{Re} z<\mu$, hence $\Sigma \geq \mu$. Moreover, taking $z=i \lambda \in i \mathbb{R}$ and using the fact that $e^{-t A}$ is a semigroup of contractions in $X$, we find

$$
\left\|(A-i \lambda)^{-1}\right\| \leq \int_{0}^{\infty}\left\|e^{-t A}\right\| \mathrm{t} \leq \int_{0}^{\infty} \min \left\{1, C e^{-\mu t}\right\} \mathrm{t}=\frac{1+\log (C)}{\mu}
$$

Taking the supremum over $\lambda \in \mathbb{R}$, we conclude that $\Psi \geq \mu /(1+\log (C))$.

ii) Conversely, to estimate the semigroup $e^{-t A}$ in terms of $\Sigma$ or $\Psi$, we use the inverse Laplace formula

$$
e^{-t A}=\frac{1}{2 \pi i} \int_{\Gamma(\mu, \alpha)}(A-z)^{-1} e^{-z t} \mathrm{Z},
$$

where $0<\mu<\Sigma$ and $\Gamma(\mu, \alpha)=\Gamma_{-}(\mu, \alpha) \cup \Gamma_{0}(\mu, \alpha) \cup \Gamma_{+}(\mu, \alpha)$ is the polygonal contour defined by

$$
\begin{aligned}
\Gamma_{0}(\mu, \alpha) & =\left\{z \in \mathbb{C} ; \operatorname{Re} z=\mu,|\arg z| \leq \frac{\pi}{2}-\alpha\right\} \\
\Gamma_{ \pm}(\mu, \alpha) & =\left\{z \in \mathbb{C} ; \operatorname{Re} z \geq \mu, \arg z= \pm\left(\frac{\pi}{2}-\alpha\right)\right\}
\end{aligned}
$$

and oriented from $\operatorname{Im} z=-\infty$ to $\operatorname{Im} z=+\infty$. Note that $\Gamma(\mu, \alpha)$ lies entirely in the resolvent set of $A$ by construction. Since $\operatorname{Re} z=\mu$ when $z \in \Gamma_{0}(\mu, \alpha)$, we easily obtain

$$
\left\|\int_{\Gamma_{0}(\mu, \alpha)}(A-z)^{-1} e^{-z t} \mathrm{Z}\right\| \leq N(A, \mu) \frac{2 \mu}{\tan \alpha} e^{-\mu t}
$$

where $N(A, \mu)=\sup \left\{\left\|(A-z)^{-1}\right\| ; \operatorname{Re}(z)=\mu\right\}$. On the other hand, any $z \in \Gamma_{+}(\mu, \alpha)$ can be parametrized as $z=x+(i x / \tan \alpha)$ with $x \geq \mu$, and the resolvent at this point can be estimated as follows:

$$
\left\|(A-z)^{-1}\right\| \leq \frac{1}{\operatorname{dist}(z, \Theta(A))} \leq \frac{1}{\operatorname{dist}\left(z, \Delta_{\alpha}\right)}=\frac{1}{x}
$$

We thus find

$$
\left\|\int_{\Gamma_{+}(\mu, \alpha)}(A-z)^{-1} e^{-z t} \mathrm{z}\right\| \leq \int_{\mu}^{\infty} \frac{1}{x} e^{-t x} \frac{\mathrm{x}}{\tan \alpha} \leq \frac{1}{\tan \alpha} \frac{e^{-\mu t}}{\mu t},
$$

and the contribution of $\Gamma_{-}(\mu, \alpha)$ is estimated in exactly the same way. Collecting these bounds are using the fact that $\left\|e^{-t A}\right\| \leq 1 \leq 1 / \tan \alpha$, we arrive at

$$
\left\|e^{-t A}\right\| \leq \frac{1}{\pi \tan \alpha}\left(\mu N(A, \mu) e^{-\mu t}+\min \left\{\pi, \frac{e^{-\mu t}}{\mu t}\right\}\right) \leq \frac{1}{\pi \tan \alpha}(\mu N(A, \mu)+2 \pi) e^{-\mu t}
$$

iii) Assume that $0<\mu<\Psi$. Using the second resolvent formula and the definition of $\Psi$, we find for all $\lambda \in \mathbb{R}$ :

$$
\left\|(A-\mu-i \lambda)^{-1}\right\| \leq \frac{\left\|(A-i \lambda)^{-1}\right\|}{1-\mu\left\|(A-i \lambda)^{-1}\right\|} \leq \frac{1}{\Psi-\mu},
$$

hence $N(A, \mu) \leq(\Psi-\mu)^{-1}$.

We next give a proof of Lemma 1.3, which illustrates the pseudospectral nature of the quantity $\Psi(\epsilon)$.

Proof of Lemma 1.3. 
i) If $\operatorname{Re}(z) \leq 0$, we know that $\left\|\left(H_{\epsilon}-z\right)^{-1}\right\| \leq 1 / \operatorname{dist}\left(z, \Theta\left(H_{\epsilon}\right)\right) \leq 1$. If $0<\operatorname{Re}(z) \leq \kappa \Psi(\epsilon)$ for some $\kappa \in(0,1)$, we have by Lemma 1.1-iii):

$$
\left\|\left(H_{\epsilon}-z\right)^{-1}\right\| \leq N\left(H_{\epsilon}, \operatorname{Re}(z)\right) \leq \frac{1}{\Psi(\epsilon)-\operatorname{Re}(z)} \leq \frac{1}{\Psi(\epsilon)} \frac{1}{1-\kappa} \leq \frac{1}{1-\kappa} .
$$

Thus $\left\|\left(H_{\epsilon}-z\right)^{-1}\right\|$ is uniformly bounded by $(1-\kappa)^{-1}$ when $\operatorname{Re}(z) \leq \kappa \Psi(\epsilon)$.

ii) We argue by contraposition. Fix $K_{0} \geq 1, N \in \mathbb{N}$, and assume that $\left\|\left(H_{\epsilon}-z\right)^{-1}\right\| \leq K_{0} \epsilon^{-N}$ whenever $\operatorname{Re}(z) \leq \mu_{\epsilon}$. We shall show that there exists $K \geq 1$ (independent of $\epsilon$ ) such that

$$
\mu_{\epsilon} \leq K \Psi(\epsilon)\left(1+\log \Psi(\epsilon)+\log \left(\epsilon^{-1}\right)\right), \quad \text { for all } \epsilon \in(0,1] .
$$

Note that (A.1) is automatically satisfied if $\mu_{\epsilon} \leq 1$, hence we assume from now on that $\mu_{\epsilon} \geq 1$. Applying Lemma 1.1 -ii) with $\mu=\mu_{\epsilon}$, and using the fact that $\alpha=\mathcal{O}(\epsilon)$ when $A=H_{\epsilon}$, we see that $\left\|e^{-t H_{\epsilon}}\right\| \leq C\left(H_{\epsilon}, \mu_{\epsilon}\right) e^{-\mu_{\epsilon} t}$ for all $t \geq 0$, where

$$
C\left(H_{\epsilon}, \mu_{\epsilon}\right) \leq \frac{1}{\pi \tan \alpha}\left(\mu_{\epsilon} K_{0} \epsilon^{-N}+2 \pi\right) \leq \mu_{\epsilon} K_{1} \epsilon^{-N-1},
$$

for some $K_{1} \geq 1$. Next, using the lower bound on $\Psi$ given by Lemma 1.1-i $\mathbf{i}$, we obtain

$$
\mu_{\epsilon} \leq \Psi(\epsilon)\left(1+\log C\left(H_{\epsilon}, \mu_{\epsilon}\right)\right) \leq \Psi(\epsilon)\left(1+\log \left(\mu_{\epsilon}\right)+\log \left(K_{1} \epsilon^{-N-1}\right)\right) .
$$

The desired bound (A.1) is now a direct consequence of the following elementary result:

Claim: If $\mu \geq 1, \Psi \geq 1$, and $C \geq 1$ satisfy $\mu \leq \Psi(C+\log \mu)$, then

$$
\mu \leq \Psi(C+2 \log 2+2 \log \Psi+\log C) .
$$

Indeed, the hypothesis implies that $\mu \leq \mu_{0}$, where $\mu_{0} \geq 1$ is uniquely determined by the relation $\mu_{0}=\Psi\left(C+\log \mu_{0}\right)$. Since $\log \mu_{0} \leq \sqrt{\mu_{0}}$, we have $\mu_{0} \leq \Psi\left(C+\sqrt{\mu_{0}}\right)$, hence

$$
\sqrt{\mu_{0}} \leq \frac{1}{2}\left(\Psi+\sqrt{\Psi^{2}+4 C \Psi}\right) \leq \Psi+\sqrt{C \Psi} .
$$

Using this bound and the fact that $\mu \leq \Psi\left(C+\log \mu_{0}\right)$, we easily obtain (A.2). This concludes the proof of the claim, hence of Lemma 1.3 .

We now briefly recall the comparison argument with semiclassical models which yields the lower bound (1.11).

Proof of Lemma 1.7. Let $V(x, \epsilon)=x^{2}+\frac{f^{\prime}(x)^{2}}{\epsilon^{2}}$. By the Min-Max principle, it is sufficent to find a constant $M_{3} \geq 1$ such that

$$
\left\langle\hat{H}_{\epsilon} u, u\right\rangle=\int_{\mathbb{R}}\left(\left|\partial_{x} u\right|^{2}+V(x, \epsilon)|u|^{2}\right) \mathrm{x} \geq \frac{\|u\|^{2}}{M_{3} \epsilon^{2 \nu}}, \quad \text { for all } u \in \mathcal{D}
$$

and for any $\epsilon \in(0,1]$ a nonzero function $\varphi_{\epsilon} \in C_{0}^{\infty}(\mathbb{R}) \subset \mathcal{D}$ such that

$$
\left\langle\hat{H}_{\epsilon} \varphi_{\epsilon}, \varphi_{\epsilon}\right\rangle \leq \frac{M_{3}}{\epsilon^{2 \nu}}\left\|\varphi_{\epsilon}\right\|^{2}
$$

Hypothesis 1.6 ensures that there exists $L>0$ such that

$$
f^{\prime}(x)^{2} \geq \frac{k^{2}}{2|x|^{2(k+1)}} \quad \text { for } \quad|x| \geq L,
$$


and that $f$ has a finite set $\left\{x_{1}, \ldots, x_{N}\right\}$ of (nondegenerate) critical points. Hence there exists a partition of unity $\sum_{j=0}^{N} \chi_{j}^{2}=1$ such that $\chi_{j} \in C_{0}^{\infty}(\mathbb{R})$ for $j \in\{1, \ldots, N\}$, supp $\chi_{0} \subset(-\infty,-L) \cup$ $(L,+\infty)$, and such that $f$ has exactly one critical point (namely, $x_{j}$ ) in the support of $\chi_{j}$ for $j \in\{1, \ldots, N\}$. The IMS localization formula of [2] provides a constant $C>0$ such that

$$
\int_{\mathbb{R}}\left(\left|\partial_{x} u\right|^{2}+V(x, \epsilon)|u|^{2}\right) \mathrm{x} \geq \sum_{j=0}^{N} \int_{\mathbb{R}}\left(\left|\partial_{x} u_{j}\right|^{2}+V(x, \epsilon)\left|u_{j}\right|^{2}\right) \mathrm{x}-C\|u\|^{2},
$$

with $u_{j}=\chi_{j} u$. By construction, for $j \in\{1, \ldots, N\}$, there exists $c_{j}>0$ such that

$$
V(x, \epsilon) \geq \frac{f^{\prime}(x)^{2}}{\epsilon^{2}} \geq \frac{c_{j}^{2}\left(x-x_{j}\right)^{2}}{\epsilon^{2}}, \quad \text { for all } x \in \operatorname{supp} \chi_{j} .
$$

Hence the comparison with the harmonic oscillator hamiltonian $-\partial_{x}^{2}+c_{j}^{2} \epsilon^{-2}\left(x-x_{j}\right)^{2}$ implies that $\left\langle\hat{H}_{\epsilon} u_{j}, u_{j}\right\rangle \geq c_{j} \epsilon^{-1}\left\|u_{j}\right\|^{2}$ for all $j \in\{1, \ldots, N\}$. For $j=0$, we notice that

$$
V(x, \epsilon) \geq x^{2}+\frac{k^{2}}{2 \epsilon^{2}|x|^{2(k+1)}} \geq c_{0} \epsilon^{-\frac{2}{k+2}}, \quad \text { for all } x \in \operatorname{supp} \chi_{0} \subset \mathbb{R} \backslash[-L, L] .
$$

Thus the first term $\left\langle\hat{H}_{\epsilon} u_{0}, u_{0}\right\rangle$ is bounded from below by $c_{0} \epsilon^{-\nu}$. Summing up all the lower bounds of the terms in (A.5), and recalling that $\|u\|^{2} \leq\left\langle\hat{H}_{\epsilon} u_{j}, u_{j}\right\rangle$, we obtain (A.3) provided $M_{3}^{-1}(1+C) \leq \min \left\{c_{j} ; 0 \leq j \leq N\right\}$. For the upper bound (A.4), it suffices to take $\varphi_{\epsilon}(x)=$ $\varphi\left(\epsilon^{\nu} x\right)$ with $\varphi \in C_{0}^{\infty}((1,2))$ and $\varphi \not \equiv 0$.

Following exactly the same lines, one can also prove the following result, which is needed in Section 3.3 :

Lemma A.1 Let $\beta: \mathbb{R} \rightarrow \mathbb{R}_{+}$be the function defined in Section 3.3 and depicted in Fig. 1 . There is a constant $M_{0}>0$ such that, for any $u \in \mathcal{D}$ and any $\epsilon \in(0,1]$,

$$
\int_{\mathbb{R}}\left(\left|\partial_{x} u\right|^{2}+x^{2}|u|^{2}+\frac{\beta}{\epsilon} f^{\prime}(x)^{2}|u|^{2}\right) x \geq \frac{M_{0}}{\epsilon^{\bar{\nu}}}\|u\|_{L^{2}}^{2}, \quad \text { where } \quad \bar{\nu}=\frac{2}{k+4} .
$$

Proof: We assume that the parameter $A>0$ entering the definition (3.18) of the function $\beta$ is large enough so that all critical points of $f$ are contained in the interval $[-A+1, A-1]$, and so that

$$
f^{\prime}(x)^{2} \geq \frac{k^{2}}{2|x|^{2(k+1)}}, \quad \text { whenever }|x| \geq A
$$

We now consider the potential $V(x, \epsilon)=x^{2}+\frac{\beta(x) f^{\prime}(x)^{2}}{\epsilon}$ and we introduce like in the proof of Lemma 1.7 a partition of unity $\sum_{j=0}^{N} \chi_{j}^{2} \equiv 1$ with $\chi_{j} \in C_{0}^{\infty}(\mathbb{R})$ for all $j \in\{1, \ldots, N\}$, $\operatorname{supp} \chi_{0} \subset(-\infty,-A) \cup(A,+\infty)$, and such that $f$ has exactly one critical point in supp $\chi_{j}$ for $j \in\{1, \ldots, N\}$. Again the IMS localization formula provides a constant $C>0$ such that

$$
(1+C) \int_{\mathbb{R}}\left(\left|\partial_{x} u\right|^{2}+V(x, \epsilon)|u|^{2}\right) \mathrm{x} \geq \sum_{j=0}^{N} \int_{\mathbb{R}}\left(\left|\partial_{x} u_{j}\right|^{2}+V(x, \epsilon)\left|u_{j}\right|^{2}\right) \mathrm{x},
$$

with $u_{j}=\chi_{j} u$. For $j \in\{1, \ldots, N\}$ we find as in the proof of Lemma 1.7 a constant $c_{j}>0$ such that

$$
\int_{\mathbb{R}}\left(\left|\partial_{x} u_{j}\right|^{2}+V(x, \epsilon)\left|u_{j}\right|^{2}\right) \mathrm{x} \geq \frac{c_{j}}{\epsilon^{1 / 2}}\left\|u_{j}\right\|^{2} .
$$


For $j=0$, we use the definition (3.18) and the condition (A.7) to find an appropriate lower bound on $V(x, \epsilon)$. If $c_{0}>0$ is sufficiently small, then for any $x \in \operatorname{supp} \chi_{0}$ we have either

$$
A \leq|x| \leq B_{\epsilon}, \quad \text { so that } \quad V(x, \epsilon) \geq x^{2}+\frac{\beta_{0} k^{2}}{2 A^{2 k} \epsilon x^{2}} \geq c_{0} \epsilon^{-1 / 2},
$$

or

$$
|x| \geq B_{\epsilon}, \quad \text { so that } \quad V(x, \epsilon) \geq x^{2}+\frac{\beta_{0} \epsilon^{-\frac{k}{k+4}} k^{2}}{2 \epsilon|x|^{2(k+1)}} \geq c_{0} \epsilon^{-\frac{2}{k+4}} .
$$

Since $\bar{\nu}=\frac{2}{k+4}<\frac{1}{2}$, it follows that $V(x, \epsilon) \geq c_{0} \epsilon^{-\bar{\nu}}$ if $x \in \operatorname{supp} \chi_{0}$, hence (A.8) holds for $j=0$ too if we replace the exponent $1 / 2$ by $\bar{\nu}$. We conclude as before.

\section{References}

[1] J. Aguilar, J.M. Combes. A class of analytic perturbations for one-body Schrödinger Hamiltonians. Comm. Math. Phys. 22 (1971), 269-279.

[2] H.L Cycon, R.G Froese, W. Kirsch, and B. Simon. Schrödinger operators with application to quantum mechanics and global geometry. Text and Monographs in Physics, Springer, 1987.

[3] E.B. Davies. Non-self-adjoint differential operators. Bull. London Math. Soc. 34 (2002), 513-532.

[4] N. Dencker, J. Sjöstrand, and M. Zworski. Pseudospectra of semi-classical (pseudo)differential operators. Comm. Pure Appl. Math. 57 (2004), 384-415.

[5] L. Desvillettes and C. Villani. On the trend to global equilibrium for spatially inhomogeneous kinetic systems: the Boltzmann equation. Invent. Math. 159 (2005), 245-316.

[6] Th. Gallay and C.E. Wayne. Invariant manifolds and the long-time asymptotics of the Navier-Stokes and vorticity equations on $\mathbb{R}^{2}$. Arch. Ration. Mech. Anal. 163 (2002), 209258.

[7] Th. Gallay and C.E. Wayne. Global stability of vortex solutions of the two-dimensional Navier-Stokes equation. Comm. Math. Phys. 255 (2005), 97-129.

[8] M. Hager and J. Sjöstrand. Eigenvalue asymptotics for randomly perturbed non-selfadjoint operators. Preprint.

[9] B. Helffer. Semi-classical analysis for the Schrödinger operator and applications. Lecture Notes in Mathematics 1336, Springer, Berlin, 1988.

[10] B. Helffer and F. Nier. Hypoelliptic estimates and spectral theory for Fokker-Planck Operators and Witten Laplacians. Lect. Notes in Math. 1862, Springer, 2005.

[11] B. Helffer and J. Nourrigat. Hypoellipticité maximale pour des opérateurs polynômes de champs de vecteur. Progress in Mathematics 58, Birkhäuser, 1985.

[12] B. Helffer and J. Sjöstrand. Résonances en limite semiclassique. Mém. Soc. Math. France (N.S.) No. 24-25 (1986).

[13] F. Hérau and F. Nier. Isotropic hypoellipticity and trend to the equilibrium for the FokkerPlanck equation with high degree potential. Arch. Ration. Mech. Anal. 171 (2004), 151-218.

[14] F. Hérau, C. Stolk and J. Sjöstrand. Semiclassical analysis for the Kramers-Fokker-Planck equation. Comm. Partial Differential Equations 30 (2005), 689-760.

[15] P.D. Hislop and I.M. Sigal. Introduction to spectral theory. With applications to Schrödinger operators. Applied Mathematical Sciences 113, Springer, 1996. 
[16] L. Hörmander. The analysis of linear partial differential operators. Springer, 1985.

[17] T. Kato. Perturbation theory for linear operators. Grundlehren der mathematischen Wissenschaften 132, Springer, 1966.

[18] A. Martinez. An introduction to semiclassical and microlocal analysis. Universitext, Springer, 2002.

[19] K. Pravda-Starov. A general result about pseudospectrum for Schrödinger operators. Proc. R. Soc. Lond. A 460 (2004), 471-477.

[20] L.P. Rothschild and E.M. Stein. Hypoelliptic differential operators and nilpotent groups. Acta Mathematica 137 (1977), 248-315.

[21] J. Sjöstrand. Singularités analytiques microlocales. Astérisque 95, 1-166, Soc. Math. France, 1982.

[22] J. Sjöstrand. Some results on non-self-adjoint operators, a survey. Preprint available on http://arxiv.org/pdf/0804.3701v1

[23] L. Trefethen. Pseudospectra of linear operators. SIAM Rev. 39 (1997), 383-406.

[24] C. Villani, Hypocoercivity, Memoirs of the AMS, to appear.

[25] C. Villani. Hypocoercive diffusion operators. International Congress of Mathematicians. Vol. III, 473-498, Eur. Math. Soc., Zürich, 2006. 